\title{
Split Casimir operator for simple Lie algebras, solutions of Yang-Baxter equations and Vogel parameters
}

\author{
A.P.Isaev ${ }^{a, b, c}$, S.O.Krivonos ${ }^{a}$ \\ a Bogoliubov Laboratory of Theoretical Physics, \\ Joint Institute for Nuclear Research, 141980 Dubna, Russia \\ ${ }^{b}$ St.Petersburg Department of Steklov Mathematical Institute of RAS, \\ Fontanka 27, 191023 St. Petersburg, Russia \\ ${ }^{c}$ Faculty of Physics, Lomonosov Moscow State University, Moscow, Russia \\ isaevap@theor.jinr.ru, krivonos@theor.jinr.ru
}

\begin{abstract}
We construct characteristic identities for the split (polarized) Casimir operators of the simple Lie algebras in defining (minimal fundamental) and adjoint representations. By means of these characteristic identities, for all simple Lie algebras we derive explicit formulae for invariant projectors onto irreducible subrepresentations in $T^{\otimes 2}$ in two cases, when $T$ is the defining and the adjoint representation. In the case when $T$ is the defining representation, these projectors and the split Casimir operator are used to explicitly write down invariant solutions of the Yang-Baxter equations. In the case when $T$ is the adjoint representation, these projectors and characteristic identities are considered from the viewpoint of the universal description of the simple Lie algebras in terms of the Vogel parameters.
\end{abstract}




\section{Contents}

1 Introduction $\quad 11$

2 Split Casimir operator for simple Lie algebras 3

2.1 General definitions .................... . . . 3

2.2 The split Casimir operator for simple Lie algebras in the adjoint representation ............................ 4

2.3 The split Casimir operator for highest weight representations . . . . . . . . 6

3 Split Casimir operator for Lie algebras of classical series

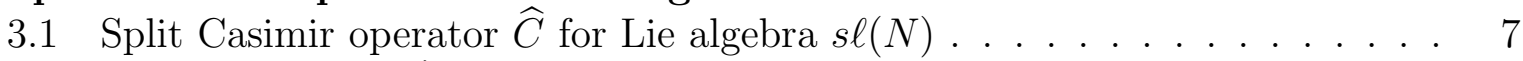

3.1.1 Operator $\widehat{C}$ for $s \ell(N)$ in the defining representation. . . . . . . . 7

3.1.2 Operator $\widehat{C}$ for $s \ell(N)$ in the adjoint representation. . . . . . . . . 9

3.2 Split Casimir operator $\widehat{C}$ for Lie algebras $s o(N)$ and $s p(2 n) \ldots . . . . . . .17$

3.2.1 Operator $\widehat{C}$ for $s o(N)$ and $s p(2 n)$ in the defining representation . . 17

3.2.2 Operator $\widehat{C}$ for $s o(N)$ and $s p(2 n)$ in the adjoint representation . . . 19

3.2 .3 The algebra $s o(8) \ldots \ldots \ldots \ldots \ldots . \ldots \ldots 22$

3.3 Universal characteristic identities for operator $\widehat{C}_{+}$in the case of Lie algebras of classical series ...................... . . . . . . . . . . .

4 Split Casimir operators $\widehat{C}$ for exceptional Lie algebras 25

4.1 Characteristic identities, projectors and $R$-matrices in the fundamental rep-

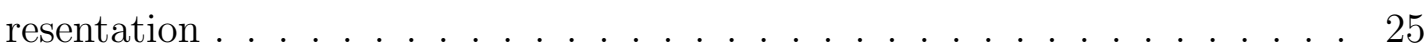

4.1.1 Basic definitions . . . . . . . . . . . . . . . 25

4.1.2 Split Casimir operator and $R$-matrix for the algebra $\mathfrak{g}_{2} \ldots \ldots$. . . 26

4.1.3 Split Casimir operator and $R$-matrix for the algebra $\mathfrak{f}_{4} \ldots \ldots \ldots$

4.1.4 Split Casimir operator and $R$-matrix for the algebra $\mathfrak{e}_{6} \ldots \ldots \ldots$

4.1.5 Split Casimir operator and $R$-matrix for the algebra $\mathfrak{e}_{7} \ldots \ldots$. . . . 32

4.1.6 Algebra $\mathfrak{e}_{8} \ldots \ldots \ldots \ldots 33$

4.2 Characteristic identities for operator $\widehat{C}$ and invariant projectors for exceptional Lie algebras in the adjoint representations. . . . . . . . . . . . . . 33

4.2.1 Basic definitions . . . . . . . . . . . . . . . . . . 33

$4.2 .2 \quad$ Algebra $\mathfrak{g}_{2} \ldots \ldots \ldots \ldots \ldots \ldots$

4.2 .3 Algebra $\mathfrak{f}_{4} \ldots \ldots \ldots \ldots \ldots$

4.2 .4 Algebra $\mathfrak{e}_{6} \ldots \ldots \ldots \ldots \ldots \ldots$

$4.2 .5 \quad$ Algebra $\mathfrak{e}_{7} \ldots \ldots \ldots \ldots \ldots \ldots \ldots$

4.2 .6 Algebra $\mathfrak{e}_{8} \ldots \ldots \ldots \ldots \ldots$

4.3 Universal characteristic identities and general comments . . . . . . . . 38

5 Universal characteristic identities for operator $\widehat{C}$ for simple Lie algebras in the adjoint representation and Vogel parameters

\section{Introduction}

It is known that the special invariant operator, the split (or polarized) Casimir operator $\widehat{C}$ (see definition in Section 2), plays an important role both in the description of the Lie 
algebras $\mathfrak{g}$ themselves and in the studies of their representation theory. On the other hand, the split Casimir operator $\widehat{C}$ is the building block (see e.g. [1], [2] and references therein) for constructing $\mathfrak{g}$-invariant solutions $r$ and $R$ of semiclassical and quantum Yang-Baxter equations

$$
\begin{gathered}
{\left[r_{12}(u), r_{13}(u+v)\right]+\left[r_{13}(u+v), r_{23}(v)\right]+\left[r_{12}(u), r_{23}(v)\right]=0,} \\
R_{12}(u) R_{13}(u+v) R_{23}(v)=R_{23}(v) R_{13}(u+v) R_{12}(u)
\end{gathered}
$$

We use here the standard matrix notation which will be explained below. Recall [3] that $\mathfrak{g}$-invariant rational solutions of the Yang-Baxter equations (1.0.2) allow one to define the Yangians $Y(\mathfrak{g})$ within the so-called RTT-realization.

In this paper, we demonstrate the usefulness of the $\mathfrak{g}$-invariant split Casimir operator $\widehat{C}$ in the representation theory of Lie algebras. Namely, for all simple Lie algebras $\mathfrak{g}$, explicit formulas are found for invariant projectors onto irreducible representations that appear in the expansion of the tensor product $T \otimes T$ of two representations $T$. These projectors are constructed in terms of the operator $\widehat{C}$ for two cases, when $T$ is the defining (minimal fundamental) and when $T \equiv$ ad is the adjoint representation of $\mathfrak{g}$.

It is natural to find such invariant projectors in terms of $\mathfrak{g}$-invariant operators, which in turn are images of special elements of the so-called centralizer algebra. The idea of this approach is not new. For example, invariant projectors acting in tensor representations of $s \ell(N)$ algebras are called Young symmetrizers and constructed as images of special elements (idempotents) of group algebra $\mathbb{C}\left[S_{r}\right]$ of the symmetric group $S_{r}$. The algebra $\mathbb{C}\left[S_{r}\right]$ centralizes the action of the $s \ell(N)$ in the space of tensors of rank $r$ (in the spaces of representations $T^{\otimes r}$ ). In this paper, we consider a very particular problem of constructing invariant projectors in representation spaces of $T^{\otimes 2}$, where $T$ is the defining, or adjoint representation but for all simple Lie algebras $\mathfrak{g}$. Our approach is closely related to the one outlined in [4]. In [4], such invariant projectors were obtained in terms of several special invariant operators and the calculations were performed using a peculiar diagram technique. In our approach, we try to construct invariant projectors in the representation space $V^{\otimes 2}$ of $T^{\otimes 2}$ by using only one $\mathfrak{g}$-invariant operator which is the split Casimir operator $\widehat{C}$. It turns out that for all simple Lie algebras $\mathfrak{g}$ in the defining representations all invariant projectors in $V^{\otimes 2}$ are constructed as polynomials in $\widehat{C}$. It is not the case for the adjoint representation, i.e. not for all algebras $\mathfrak{g}$ the invariant projectors in $V_{\mathrm{ad}}^{\otimes 2}$ are constructed as polynomials of only one operator $\widehat{C}_{\text {ad }} \equiv \operatorname{ad}^{\otimes 2} \widehat{C}$. Namely, in the case of $s \ell(N)$ and $s o(8)$ algebras there are additional $\mathfrak{g}$-invariant operators which are independent of $\widehat{C}_{\text {ad }}$ and act, respectively, in the antisymmetrized and symmetrized parts of the space $V_{\mathrm{ad}}^{\otimes 2}$. We construct such additional operators explicitly in Sections 3.1 .2 and 3.2.1.

Our study of the split Casimir operator $\widehat{C}$ was motivated by the works [13], [14] and [15], and by the idea of finding formulas for solutions of the Yang-Baxter equation expressed in terms of only the operator $\widehat{C}$. For defining (minimal fundamental) representations of the simple Lie algebras $\mathfrak{g}$ (except for the algebra $\mathfrak{e}_{8}$ ), such formulas were derived in this paper (see equations (3.1.16), (3.2.12), (4.1.29), (4.1.44), (4.1.51) and (4.1.58) below). Note that these formulas are obtained by using well-known [26], [27] spectral decompositions for rational $R$-matrices 1 . For the adjoint representations of the simple Lie algebras $\mathfrak{g}$, as it was argued in [33], there are no such formulas (we need to

\footnotetext{
${ }^{1}$ All these spectral decompositions can also be obtained from the spectral decompositions of trigonometric $R$-matrices (see e.g. [2] (section 7.2.4), [28], 29]) in the special limit $q \rightarrow 1$.
} 
extend the adjoint representations of algebras $\mathfrak{g}$; see [34], [35]). However, in the case of the adjoint representation of $\mathfrak{g}$, the knowledge of the characteristic identities for $\widehat{C}_{\mathrm{ad}}$ turns out to be a key point for understanding the so-called universal formulation of the simple Lie algebras [11] (see also the historical notes in [4], section 21.2). Though some characteristic identities and formulas for certain $\mathfrak{g}$-invariant projectors can be found in a different form in [4], we believe that the methods we used and the results obtained can be useful for future research, e.g. from the viewpoint of technical applications of the split Casimir operator.

In our paper, to simplify the notation, we everywhere write $s \ell(N), s o(N)$ and $s p(2 n)$ instead of $s \ell(N, \mathbb{C}), s o(N, \mathbb{C})$ and $s p(2 n, \mathbb{C})$.

\section{Split Casimir operator for simple Lie algebras}

\subsection{General definitions}

Let $\mathfrak{g}$ be a simple Lie algebra with the basis $X_{a}$ and defining relations

$$
\left[X_{a}, X_{b}\right]=C_{a b}^{d} X_{d}
$$

where $C_{a b}^{d}$ are the structure constants. The Cartan-Killing metric is defined in the standard way

$$
\mathrm{g}_{a b} \equiv C_{a c}^{d} C_{b d}^{c}=\operatorname{Tr}\left(\operatorname{ad}\left(X_{a}\right) \cdot \operatorname{ad}\left(X_{b}\right)\right),
$$

where ad denotes adjoint representation: $\operatorname{ad}\left(X_{a}\right)_{b}^{d}=C_{a b}^{d}$. Recall that the structure constants $C_{a b c} \equiv C_{a b}^{d} \mathrm{~g}_{d c}$ are antisymmetric under permutation of indices $(a, b, c)$. We denote an enveloping algebra of the Lie algebra $\mathfrak{g}$ as $\mathcal{U}(\mathfrak{g})$. Let $\mathfrak{g}^{d f}$ be the inverse matrix to the Cartan-Killing metric (2.1.2). We use this matrix and construct the operator

$$
\widehat{C}=\mathfrak{g}^{a b} X_{a} \otimes X_{b} \in \mathfrak{g} \otimes \mathfrak{g} \subset \mathcal{U}(\mathfrak{g}) \otimes \mathcal{U}(\mathfrak{g})
$$

which is called the split (or polarized) Casimir operator of the Lie algebra $\mathfrak{g}$. This operator is related to the usual quadratic Casimir operator

$$
C_{(2)}=\mathrm{g}^{a b} X_{a} \cdot X_{b} \in \mathcal{U}(\mathfrak{g})
$$

by means of the formula

$$
\Delta\left(C_{(2)}\right)=C_{(2)} \otimes I+I \otimes C_{(2)}+2 \widehat{C}
$$

where $\Delta$ is the standard comultiplication for enveloping algebras $\mathcal{U}(\mathfrak{g})$ :

$$
\Delta\left(X_{a}\right)=\left(X_{a} \otimes I+I \otimes X_{a}\right)
$$

The following statement holds (see, for example, [7], [9]).

Proposition 2.1.1 The operator $\widehat{C}$, given in (2.1.3), does not depend on the choice of the basis in $\mathfrak{g}$ and satisfies the condition (which is called ad-invariance or $\mathfrak{g}$-invariance):

$$
[\Delta(A), \widehat{C}]=[(A \otimes I+I \otimes A), \widehat{C}]=0, \quad \forall A \in \mathfrak{g}
$$


where $\Delta$ is comultiplication (2.1.6). In addition, the operator $\widehat{C}$ obeys the equations

$$
\left[\widehat{C}_{12}, \widehat{C}_{13}+\widehat{C}_{23}\right]=0 \Rightarrow\left[\widehat{C}_{13}, \widehat{C}_{23}\right]=\frac{1}{2}\left[\widehat{C}_{12}, \widehat{C}_{13}-\widehat{C}_{23}\right]
$$

which use the standard notation

$$
\widehat{C}_{12}=\mathrm{g}^{a b} X_{a} \otimes X_{b} \otimes I, \quad \widehat{C}_{13}=\mathrm{g}^{a b} X_{a} \otimes I \otimes X_{b}, \quad \widehat{C}_{23}=\mathrm{g}^{a b} I \otimes X_{a} \otimes X_{b} .
$$

Here $I$ is the unit element in $\mathcal{U}(\mathfrak{g})$ and $\widehat{C}_{i j} \in \mathcal{U}(\mathfrak{g}) \otimes \mathcal{U}(\mathfrak{g}) \otimes \mathcal{U}(\mathfrak{g})$.

Relations (2.1.8) indicate that the split Casimir operator (2.1.3) realizes the KonoDrinfeld Lie algebra and can be used as a building block for constructing solutions to the quasi-classical (1.0.1) and quantum (1.0.2) Yang-Baxter equations. In particular, the solution for the quasi-classical Yang-Baxter equation (1.0.1) is the operator $r(u)=\widehat{C} / u$ (see e.g. [2]).

Remark. Let the normalization of the generators $H_{i}, E_{\alpha}$ in the Cartan-Weyl basis of the algebra $\mathfrak{g}$ be chosen so that we have for (2.1.4) and (2.1.3):

$$
C_{(2)}=g^{i j}\left(H_{i} H_{j}\right)+\sum_{\alpha}\left(E_{\alpha} E_{-\alpha}\right) \Rightarrow \widehat{C}=g^{i j}\left(H_{i} \otimes H_{j}\right)+\sum_{\alpha}\left(E_{\alpha} \otimes E_{-\alpha}\right),
$$

where the sum goes over all roots $\alpha$ and $g^{i j}$ is the inverse matrix to the metric in the root space

$$
g_{i j}=\sum_{\alpha} \alpha_{i} \alpha_{j}
$$

Then the split Casimir operator is decomposed into the sum $\widehat{C}=\left(r_{+}+r_{-}\right)$of two solutions $r=r_{+}$and $r=r_{-}$of a constant (i.e. independent of the spectral parameter) semiclassical Yang-Baxter equation $\left[r_{12}, r_{13}\right]+\left[r_{12}, r_{23}\right]+\left[r_{13}, r_{23}\right]=0$. These solutions are written in the form (see e.g. [1],[2])

$$
r_{+}=\frac{1}{2} g^{i j}\left(H_{i} \otimes H_{j}\right)+\sum_{\alpha>0}\left(E_{\alpha} \otimes E_{-\alpha}\right), \quad r_{-}=\frac{1}{2} g^{i j}\left(H_{i} \otimes H_{j}\right)+\sum_{\alpha>0}\left(E_{-\alpha} \otimes E_{\alpha}\right),
$$

where the sum goes over all positive roots $\alpha>0$ of the algebra $\mathfrak{g}$.

\subsection{The split Casimir operator for simple Lie algebras in the adjoint representation}

The generators $X_{a}$ of a simple Lie algebra $\mathfrak{g}$ satisfy the defining relations (2.1.1) and, in the adjoint representation, $X_{a}$ are implemented as matrices $\operatorname{ad}\left(X_{a}\right)_{b}^{d}=C_{a b}^{d}$. In this case the split Casimir operator (2.1.3) is written as

$$
\left(\widehat{C}_{\mathrm{ad}}\right)_{b_{1} b_{2}}^{a_{1} a_{2}} \equiv(\mathrm{ad} \otimes \mathrm{ad})_{b_{1} b_{2}}^{a_{1} a_{2}}(\widehat{C})=C_{h b_{1}}^{a_{1}} C_{f b_{2}}^{a_{2}} \mathrm{~g}^{h f} .
$$

By definition this operator satisfies identities (2.1.8). Below we need one more ad-invariant operator

$$
(\mathbf{K})_{b_{1} b_{2}}^{a_{1} a_{2}}=\mathrm{g}^{a_{1} a_{2}} \mathrm{~g}_{b_{1} b_{2}} .
$$


The operators (2.2.1) and (2.2.2) act in the tensor product $V_{\text {ad }} \otimes V_{\text {ad }}$ of two spaces $V_{\text {ad }}=\mathfrak{g}$ of the adjoint representation and have the symmetry properties $\left(\widehat{C}_{\mathrm{ad}}\right)_{b_{1} b_{2}}^{a_{1} a_{2}}=\left(\widehat{C}_{\mathrm{ad}}\right)_{b_{2} b_{1}}^{a_{2} a_{1}}$ and $\mathbf{K}_{b_{1} b_{2}}^{a_{1} a_{2}}=\mathbf{K}_{b_{2} b_{1}}^{a_{2} a_{1}}$, which are conveniently written in the form

$$
\left(\widehat{C}_{\mathrm{ad}}\right)_{21}=\mathbf{P}\left(\widehat{C}_{\mathrm{ad}}\right)_{12} \mathbf{P}=\left(\widehat{C}_{\mathrm{ad}}\right)_{12}, \quad \mathbf{K}_{21}=\mathbf{P} \mathbf{K}_{12} \mathbf{P}=\mathbf{K}_{12},
$$

where 1,2 are numbers of spaces $V_{\text {ad }}$ in the product $\left(V_{\text {ad }} \otimes V_{\text {ad }}\right)$ and $\mathbf{P}$ is permutation matrix in $\left(V_{\mathrm{ad}} \otimes V_{\mathrm{ad}}\right)$ :

$$
\mathbf{P}\left(X_{a_{1}} \otimes X_{a_{2}}\right)=\left(X_{a_{2}} \otimes X_{a_{1}}\right)=\left(X_{b_{1}} \otimes X_{b_{2}}\right) \mathbf{P}_{a_{1} a_{2}}^{b_{1} b_{2}}, \quad \mathbf{P}_{a_{1} a_{2}}^{b_{1} b_{2}}=\delta_{a_{2}}^{b_{1}} \delta_{a_{1}}^{b_{2}} .
$$

Here $\left(X_{a} \otimes X_{b}\right)$ is the basis in the space $\left(V_{\mathrm{ad}} \otimes V_{\mathrm{ad}}\right)$. Define the symmetrized and antisymmetrized parts of the operator $\widehat{C}_{\mathrm{ad}}$

$$
\left(\widehat{C}_{ \pm}\right)_{b_{1} b_{2}}^{a_{1} a_{2}}=\frac{1}{2}\left(\left(\widehat{C}_{\mathrm{ad}}\right)_{b_{1} b_{2}}^{a_{1} a_{2}} \pm\left(\widehat{C}_{\mathrm{ad}}\right)_{b_{1} b_{2}}^{a_{2} a_{1}}\right), \quad \widehat{C}_{ \pm}=\mathbf{P}_{ \pm}^{(a d)} \widehat{C}_{\mathrm{ad}}=\widehat{C}_{\mathrm{ad}} \mathbf{P}_{ \pm}^{(a d)}
$$

where $\mathbf{P}_{ \pm}^{(a d)}=\frac{1}{2}(\mathbf{I} \pm \mathbf{P})$ and $\mathbf{I}$ is the unit operator in $\left(V_{\mathrm{ad}}\right)^{\otimes 2}$.

Proposition 2.2.1 The operators $\widehat{C}_{\mathrm{ad}}, \widehat{C}_{ \pm}$and $\mathbf{K}$, given in (2.2.1), (2.2.2) and 2.2.4), satisfy the identities

$$
\begin{gathered}
\widehat{C}_{-}^{2}=-\frac{1}{2} \widehat{C}_{-}, \\
\widehat{C}_{-} \mathbf{K}=0=\mathbf{K} \widehat{C}_{-}, \quad \widehat{C}_{\mathrm{ad}} \mathbf{K}=\mathbf{K} \widehat{C}_{\mathrm{ad}}=-\mathbf{K}, \\
\widehat{C}_{+} \mathbf{K}=\mathbf{K} \widehat{C}_{+}=-\mathbf{K} .
\end{gathered}
$$

Proof. To prove equality (2.2.5), we note that $\widehat{C}_{-}$has a useful expression followed from the Jacobi identity $C_{a b}^{d} C_{d c}^{r}+\operatorname{cycle}(a, b, c)=0$ (see e.g. [4]):

$$
\left(\widehat{C}_{-}\right)_{b_{1} b_{2}}^{a_{1} a_{2}}=-\frac{1}{2} C_{d}^{a_{1} a_{2}} C_{b_{1} b_{2}}^{d}, \quad C_{d}^{a_{1} a_{2}} \equiv C_{d b_{2}}^{a_{1}} \mathrm{~g}^{b_{2} a_{2}}
$$

Using this expression and identities

$$
C_{b_{1} b_{2}}^{d} C_{a}^{b_{1} b_{2}}=\delta_{a}^{d} \quad \Leftrightarrow \quad \operatorname{ad}\left(C_{(2)}\right)_{r}^{f}=\mathrm{g}^{a b} C_{a d}^{f} C_{b r}^{d}=\delta_{r}^{f}
$$

which are equivalent to the definition (2.1.2) of the Cartan-Killing metric, we calculate $\widehat{C}_{-}^{2}$ and obtain (2.2.5). The first equality in (2.2.6) follows from the evident relations $(\mathbf{I}-\mathbf{P}) \mathbf{K}=0=\mathbf{K}(\mathbf{I}-\mathbf{P})$. The second equality in (2.2.6) is proved with the help of identities (2.2.9) and complete antisymmetry of the constants $C_{a b c}=C_{a b}^{d} \mathrm{~g}_{d c}$. Relations (2.2.7) are derived from (2.2.6).

Now we take into account definitions (2.2.1), (2.2.2), (2.2.3) and relations (2.2.5), (2.2.8), (2.2.9), and $C_{b a}^{a}=0$, which is valid for all simple Lie algebras, and obtain general formulas for the traces

$$
\begin{gathered}
\operatorname{Tr}\left(\widehat{C}_{\mathrm{ad}}\right)=0, \quad \operatorname{Tr}\left(\widehat{C}_{ \pm}\right)= \pm \frac{1}{2} \operatorname{dim} \mathfrak{g}, \quad \operatorname{Tr}\left(\widehat{C}_{\mathrm{ad}}^{2}\right)=\operatorname{dim} \mathfrak{g} \\
\operatorname{Tr}\left(\widehat{C}_{-}^{2}\right)=-\frac{1}{2} \operatorname{Tr}\left(\widehat{C}_{-}\right)=\frac{1}{4} \operatorname{dim} \mathfrak{g}, \quad \operatorname{Tr}\left(\widehat{C}_{+}^{2}\right)=\operatorname{Tr}\left(\widehat{C}_{\mathrm{ad}}^{2}-\widehat{C}_{-}^{2}\right)=\frac{3}{4} \operatorname{dim} \mathfrak{g} \\
\operatorname{Tr}(\mathbf{K})=\operatorname{dim} \mathfrak{g}, \quad \operatorname{Tr}(\mathbf{I})=(\operatorname{dim} \mathfrak{g})^{2}, \quad \operatorname{Tr}(\mathbf{P})=\operatorname{dim} \mathfrak{g} .
\end{gathered}
$$


where $\operatorname{Tr} \equiv \operatorname{Tr}_{1} \operatorname{Tr}_{2}$ is the trace in the space $V_{\text {ad }} \otimes V_{\text {ad }}$ (indices 1 and 2 are attributed to factors in the product $\left.V_{\mathrm{ad}} \otimes V_{\mathrm{ad}}\right)$. These formulas will be used in what follows.

Using the characteristic identity (2.2.5) for the operator $\widehat{C}_{-}$, one can construct two mutually orthogonal projectors

$$
\mathrm{P}_{1}=-2 \widehat{C}_{-}, \quad \mathrm{P}_{2}=2 \widehat{C}_{-}+\mathbf{P}_{-}^{(\mathrm{ad})} \quad \Rightarrow \quad \mathrm{P}_{i} \mathrm{P}_{k}=\mathrm{P}_{i} \delta_{i k}
$$

which decompose the antisymmetrized part $\mathbf{P}_{-}^{(a d)}(\operatorname{ad} \otimes \operatorname{ad})$ of the representation $(\operatorname{ad} \otimes \operatorname{ad})$ into two subrepresentations $\mathrm{X}_{1,2}=\mathrm{P}_{1,2}(\mathrm{ad} \otimes \mathrm{ad})$. Dimensions of these subrepresentations are equal to the traces of corresponding projectors (2.2.11)

$$
\operatorname{dim} \mathrm{X}_{1}=\operatorname{Tr}\left(\mathrm{P}_{1}\right)=\operatorname{dim} \mathfrak{g}, \quad \operatorname{dim} \mathrm{X}_{2}=\operatorname{Tr}\left(\mathrm{P}_{2}\right)=\frac{1}{2} \operatorname{dim} \mathfrak{g}(\operatorname{dim} \mathfrak{g}-3)
$$

where we use the general formulae (2.2.10). Since the constants $C_{b_{1} b_{2}}^{d}$ play the role of the Clebsch-Gordan coefficients for the fusion $\mathrm{ad}^{\otimes 2} \rightarrow \mathrm{ad}$, we see from the explicit form (2.2.8) of the operator $\widehat{C}_{-}$that the projector $\mathrm{P}_{1}$, given in (2.2.11), extracts the adjoint representation $\mathbf{X}_{1}=$ ad in $\mathbf{P}_{-}^{(a d)}\left(\operatorname{ad}^{\otimes 2}\right)$. Thus, the adjoint representation is always contained in the antisymmetrized part $\mathbf{P}_{-}^{(a d)}\left(\operatorname{ad}^{\otimes 2}\right)$. The first formula in (2.2.12) confirms the equivalence of $X_{1}$ and ad. Note also that $X_{2}$ is not necessarily irreducible representation for all simple Lie algebras. As we will see below (see Remark after Proposition [3.1.2), the representation $\mathrm{X}_{2}$ is reducible for algebras of the series $A_{n}=s \ell(n+1)$.

\subsection{The split Casimir operator for highest weight representa- tions}

The invariant metric for a simple Lie algebra $\mathfrak{g}$ is uniquely determined up to a normalization constant (see e.g. [7]), i.e. such metric is always proportional to the Cartan-Killing metric (2.1.2). Therefore, for any irreducible representation $T$ of the simple Lie algebra $\mathfrak{g}$ we have

$$
\operatorname{Tr}\left(T\left(X_{a}\right) \cdot T\left(X_{b}\right)\right)=\mathrm{d}_{2}(T) \mathrm{g}_{a b},
$$

where the coefficient $d_{2}(T)$ characterizes the representation $T$. Indeed, $d_{2}(T)$ is expressed in terms of values $c_{2}^{(T)}$ of the quadratic Casimir (2.1.4) in the irreducible representation of $T$ using the well-known relation

$$
c_{2}^{(T)} \operatorname{dim}(T)=\mathrm{d}_{2}(T) \operatorname{dim}(\mathfrak{g})
$$

which is obtained from (2.3.1) by contraction with the inverse metric $\mathrm{g}^{a b}$.

Let $T_{\lambda_{1}}$ and $T_{\lambda_{2}}$ be two irreducible representations with the highest weights $\lambda_{1}$ and $\lambda_{2}$ acting in the spaces $\mathcal{V}_{\lambda_{1}}$ and $\mathcal{V}_{\lambda_{2}}$. Let the representation $T_{\lambda_{1}} \otimes T_{\lambda_{2}}$ be decomposed into irreducible representations $T_{\lambda}$ with the highest weights $\lambda$ as follows: $T_{\lambda_{1}} \otimes T_{\lambda_{2}}=\sum_{\lambda} n_{\lambda} T_{\lambda}$, where $n_{\lambda}$ is the multiplicity of occurrence of $T_{\lambda}$ in the expansion of $T_{\lambda_{1}} \otimes T_{\lambda_{2}}$. Denote the space of the representation $T_{\lambda}$ as $\mathcal{V}_{\lambda}$. Then, from (2.1.5) and expansion $\mathcal{V}_{\lambda_{1}} \otimes \mathcal{V}_{\lambda_{2}}=$ $\sum_{\lambda} n_{\lambda} \mathcal{V}_{\lambda}$, we obtain

$$
\begin{gathered}
T_{\left(\lambda_{1} \times \lambda_{2}\right)}(\widehat{C}) \cdot\left(\mathcal{V}_{\lambda_{1}} \otimes \mathcal{V}_{\lambda_{2}}\right)=\frac{1}{2} \sum_{\lambda} n_{\lambda}\left(c_{2}^{(\lambda)}-c_{2}^{\left(\lambda_{1}\right)}-c_{2}^{\left(\lambda_{2}\right)}\right) \mathcal{V}_{\lambda} \Leftrightarrow \\
T_{\left(\lambda_{1} \times \lambda_{2}\right)}(\widehat{C}) \cdot \mathcal{V}_{\lambda}=\frac{1}{2}\left(c_{2}^{(\lambda)}-c_{2}^{\left(\lambda_{1}\right)}-c_{2}^{\left(\lambda_{2}\right)}\right) \mathcal{V}_{\lambda}
\end{gathered}
$$


where we use the concise notation $T_{\left(\lambda_{1} \times \lambda_{2}\right)}:=\left(T_{\lambda_{1}} \otimes T_{\lambda_{2}}\right)$. Here $c_{2}^{(\lambda)}$ is the value of the quadratic Casimir operator $C_{(2)}$, defined in (2.1.10), in the representation with the highest weight $\lambda$

$$
c_{2}^{(\lambda)}=(\lambda, \lambda+2 \delta), \quad \delta:=\sum_{f=1}^{r} \lambda_{(f)}=\frac{1}{2} \sum_{\alpha>0} \alpha,
$$

$\lambda_{(f)}$ are the fundamental weights of the rank $r$ Lie algebra $\mathfrak{g}, \alpha$ are the roots of $\mathfrak{g}$ and summation is over positive roots $(\alpha>0)$. Note that the operator $T(\widehat{C})$ is diagonalizable for simple Lie algebras and in general its spectrum is degenerate. Therefore, formula (2.3.2) implies the characteristic identity

$$
\prod_{\lambda}^{\prime}\left(T_{\left(\lambda_{1} \times \lambda_{2}\right)}(\widehat{C})-\hat{c}_{\lambda_{1}, \lambda_{2}}^{\lambda}\right)=0, \quad \hat{c}_{\lambda_{1}, \lambda_{2}}^{\lambda}:=\frac{1}{2}\left(c_{2}^{(\lambda)}-c_{2}^{\left(\lambda_{1}\right)}-c_{2}^{\left(\lambda_{2}\right)}\right),
$$

where the prime in $\prod_{\lambda}^{\prime}$ means that the product does not run over all weights $\lambda$ that participate in the expansion: $T_{\lambda_{1}} \otimes T_{\lambda_{2}}=\sum_{\lambda} n_{\lambda} T_{\lambda}$ but only those $\lambda$ that correspond to unequal eigenvalues $\hat{c}_{\lambda_{1}, \lambda_{2}}^{\lambda}$.

In the next sections, we obtain explicit expressions for the split Casimir operator $T_{\left(\lambda_{1} \times \lambda_{2}\right)}(\widehat{C}) \equiv\left(T_{\lambda_{1}} \otimes T_{\lambda_{2}}\right)(\widehat{C})$ for all simple Lie algebras in the case when both representations $T_{\lambda_{1}}$ and $T_{\lambda_{2}}$ are either defining or adjoint. In the case when $T_{\lambda_{1}}$ and $T_{\lambda_{2}}$ are adjoint representations $\lambda_{1}=\lambda_{2}=\lambda_{\text {ad }}$, the characteristic identity (2.3.4) takes the form

$$
\begin{gathered}
\prod_{\lambda}^{\prime}\left(\operatorname{ad}^{\otimes 2}(\widehat{C})-\frac{1}{2}\left(c_{2}^{(\lambda)}-2 c_{2}^{\left(\lambda_{\mathrm{ad}}\right)}\right)\right) \equiv \prod_{\lambda}^{\prime}\left(\operatorname{ad}^{\otimes 2}(\widehat{C})-\hat{c}_{2}^{(\lambda)}\right)=0, \\
\hat{c}_{2}^{(\lambda)}:=\frac{1}{2}\left(c_{2}^{(\lambda)}-2 c_{2}^{\left(\lambda_{\mathrm{ad}}\right)}\right)=\frac{1}{2} c_{2}^{(\lambda)}-1,
\end{gathered}
$$

where $\lambda_{\text {ad }}$ is the highest weight of the adjoint representation of the algebra $\mathfrak{g}$, which is equal to the highest root $\theta$ of $\mathfrak{g}$. In the definition (2.3.6) of values $\hat{c}_{2}^{(\lambda)}$ of the operator $\operatorname{ad}^{\otimes 2}(\widehat{C})$ we use the condition (see (2.2.9))

$$
c_{2}^{\left(\lambda_{\mathrm{ad}}\right)} \equiv c_{2}^{(\theta)}=1
$$

Note that this formula is consistent with (2.3.3) only if the metric in the root space of the algebra $\mathfrak{g}$ is given by (2.1.11), which corresponds to the condition

$$
(\theta, \theta)=t^{-1}
$$

where $t$ is the dual coxeter number of the algebra $\mathfrak{g}$. In the next sections, we demonstrate this fact explicitly and also find an explicit form for the characteristic identities (2.3.5) for all finite-dimensional simple Lie algebras.

\section{Split Casimir operator for Lie algebras of classical series}

\subsection{Split Casimir operator $\widehat{C}$ for Lie algebra $s \ell(N)$}

\subsubsection{Operator $\widehat{C}$ for $s \ell(N)$ in the defining representation.}

In this Subsection, to fix the notation, we give the standard definitions of the Lie algebra $s \ell(N):=s \ell(N, \mathbb{C})$ and the corresponding operator $\widehat{C}$ in the defining representation. One can find these definitions in many monographs and textbooks (see e.g. [4], [7]). 
We denote the space $\mathbb{C}^{N}$ of the defining representation $T$ of the algebra $s \ell(N)$ as $V_{N}$. Choose the basis in $s \ell(N)$ consisting of traceless matrices

$$
T_{i j}=e_{i j}-\delta_{i j} I_{N} / N \quad \Rightarrow \quad T_{i j}=e_{i j} \quad(i \neq j), \quad T_{i i}=e_{i i}-I_{N} / N,
$$

where $e_{i j}$ are $N \times N$ matrix units, and $I_{N}$ is the unit $N \times N$ matrix. The elements (3.1.1) satisfy the defining relations

$$
\left[T_{i j}, T_{k m}\right]=\delta_{j k} T_{i m}-\delta_{i m} T_{k j} \equiv C_{i j, k m}^{r s} T_{r s}
$$

where for structure constants we have the explicit expression

$$
C_{i j, k m}^{r s}=\delta_{j k} \delta_{i}^{r} \delta_{m}^{s}-\delta_{i m} \delta_{k}^{r} \delta_{j}^{s} .
$$

Using this expression and definition (2.1.2), we find the Cartan-Killing metric for $s \ell(N)$ :

$$
\mathrm{g}_{i j, k \ell}=C_{i j, m n}^{r s} C_{k \ell, r s}^{m n}=2\left(N \delta_{j k} \delta_{i \ell}-\delta_{i j} \delta_{k \ell}\right)=\mathrm{g}_{k \ell, i j},
$$

and, for the basis (3.1.1) in the defining representation, equality (2.3.1) gives

$$
\operatorname{Tr}\left(T_{i j} T_{k \ell}\right)=\frac{1}{2 N} \mathrm{~g}_{i j, k \ell} \quad \Rightarrow \quad \mathrm{d}_{2}(T)=\frac{1}{2 N} .
$$

The inverse to (3.1.4) metric $\mathrm{g}^{i j, k \ell}$ is defined by the traceless conditions $\mathrm{g}^{i i, k \ell}=0=\mathrm{g}^{i j, k k}$ and relations

$$
\mathrm{g}_{m n, i j} \mathrm{~g}^{i j, k \ell}=\bar{I}_{m n}^{k \ell}, \quad \bar{I}_{m n}^{k \ell} \equiv \delta_{m}^{k} \delta_{n}^{\ell}-\frac{1}{N} \delta_{m n} \delta^{k \ell},
$$

where the projector $\bar{I}_{m n}^{k \ell}$ plays the role of the identity operator in the adjoint representation space $V_{\text {ad }}$; we identify $V_{\text {ad }}$ with the subspace of traceless tensors in $V_{N} \otimes V_{N}$, i.e. $V_{\text {ad }}=$ $\bar{I} \cdot V_{N}^{\otimes 2}$. As a result, we obtain

$$
\mathrm{g}^{i j, k \ell}=\frac{1}{2 N}\left(\delta^{j k} \delta^{i \ell}-\frac{1}{N} \delta^{i j} \delta^{k \ell}\right)=\mathrm{g}^{k \ell, i j} .
$$

Remark. Strictly speaking, $V_{\text {ad }}$ is the subspace of second rank traceless tensors in $V_{N} \otimes$ $\bar{V}_{N}$, where $\bar{V}_{N}$ is the space of the contragradient representation of $s \ell(N)$. In other words, $V_{\text {ad }}$ is the space of tensors with the components $\psi_{k}^{i}$ that satisfy the traceless property $\psi_{i}^{i}=0$. Further, for technical reasons, we treat $\bar{V}_{N}$ as $V_{N}$ and consider $V_{\text {ad }}$ as the space of traceless tensors in $V_{N} \otimes V_{N}$ with the components $\psi^{i k}$ such that $\psi^{i i}=0$. The cases where the difference between $V_{N}$ and $\bar{V}_{N}$ is important are specially negotiated.

The matrix $T^{\otimes 2}(\widehat{C})$ for the split Casimir operator (2.1.3) of the algebra $\operatorname{sl}(N)$ with basis (3.1.1) in the defining representation $T$ is written as

$$
T^{\otimes 2}(\widehat{C})_{j_{1} j_{2}}^{i_{1} i_{2}}=\mathrm{g}^{i j, k \ell}\left(T_{i j} \otimes T_{k \ell}\right)_{j_{1} j_{2}}^{i_{1} i_{2}}=\mathrm{g}^{i j, k \ell}\left(T_{i j}\right)_{j_{1}}^{i_{1}}\left(T_{k \ell}\right)_{j_{2}}^{i_{2}}=\frac{1}{2 N}\left(\delta_{j_{2}}^{i_{1}} \delta_{j_{1}}^{i_{2}}-\frac{1}{N} \delta_{j_{1}}^{i_{1}} \delta_{j_{2}}^{i_{2}}\right)
$$

and in the index-free notation we have

$$
T^{\otimes 2}(\widehat{C})=\frac{1}{2 N}\left(P-\frac{1}{N} I\right) \equiv \widehat{C}_{T},
$$

where $I=I_{N}^{\otimes 2}$ is the unit operator in $V_{N}^{\otimes 2}$ and $P$ is the permutation operator acting in the space $V_{N}^{\otimes 2}$. Let $e_{i}(i=1, \ldots, N)$ be the basis vector in $V_{N}$, then the operator $P$ is defined as follows

$$
P(v \otimes u)=u \otimes v \quad\left(\forall v, u \in V_{N}\right) \Rightarrow P\left(e_{k} \otimes e_{m}\right)=e_{m} \otimes e_{k}=\left(e_{i} \otimes e_{j}\right) P_{k m}^{i j},
$$


i.e. the operator $P$ has the components $P^{i j}{ }_{k m}=\delta_{m}^{i} \delta_{k}^{j}$ in the basis $\left(e_{m} \otimes e_{k}\right) \in V_{N} \otimes V_{N}$. We note that in view of (3.1.6) the equality holds

$$
\operatorname{dim}_{\mathbb{C}}(s \ell(N)) \equiv \mathrm{g}^{i j, k \ell} \mathrm{g}_{i j, k \ell}=\left(N^{2}-1\right) .
$$

In addition, setting $j_{1}=i_{2}$ in (3.1.8) and summing over $i_{2}$, we obtain the value of the quadratic Casimir operator in the defining representation. Besides, setting $j_{1}=i_{2}$ in (3.1.8) and summing over $i_{2}$, we obtain the value of the quadratic Casimir operator in the defining representation

$$
T\left(C_{(2)}\right)=\mathrm{g}^{i j, k \ell}\left(T_{i j} T_{k \ell}\right)_{j_{2}}^{i_{1}}=\frac{N^{2}-1}{2 N^{2}} \delta_{j_{2}}^{i_{1}} \Rightarrow c_{2}^{(T)}=\frac{N^{2}-1}{2 N^{2}} .
$$

This corresponds to (2.3.3) if we fix metric (2.1.11) in the root space of the algebra $s \ell(N)$ so that the square of the lengths of all roots of $s \ell(N)$ is equal to $1 / N$; in particular for the highest root $\theta:=\lambda_{\text {ad }}$ we also have (cf. (2.3.8) $)$

$$
(\theta, \theta)=1 / N
$$

Finally, for the split Casimir operator (3.1.9) of the algebra $s \ell(N)$ in the defining representation we obtain the characteristic identity

$$
\widehat{C}_{T}^{2}+\frac{1}{N^{2}} \widehat{C}_{T}+\frac{1-N^{2}}{4 N^{4}}=0 \Leftrightarrow\left(\widehat{C}_{T}+\frac{1+N}{2 N^{2}}\right)\left(\widehat{C}_{T}+\frac{1-N}{2 N^{2}}\right)=0,
$$

here and below the identity operator $I$ in $V_{N} \otimes V_{N}$ is replaced with 1 for simplicity. Identity (3.1.14) is consistent with formula (2.3.4) when the root space metric is normalized in accordance with (3.1.13). In view of (3.1.14) the projectors onto eigen-spaces of the operator $\widehat{C}_{T}$ in $V_{N} \otimes V_{N}$ have the form

$$
P_{ \pm}= \pm\left(N \widehat{C}_{T}+\frac{1 \pm N}{2 N}\right)=\frac{1}{2}(1 \pm P)
$$

where $P_{+}$and $P_{-}$denote a symmetrizer and an antisymmetrizer, respectively. Finally, the $s \ell(N)$-symmetric solution of the Yang-Baxter equation (1.0.2) in the defining representation (which is called the Yang solution) is written in several equivalent ways (including the form of $R(u)$ written in terms of the operator $\widehat{C}_{T}$ ):

$$
R(u)=\frac{u+P}{1-u}=\frac{(u+1)}{(1-u)} P_{+}-P_{-} \Leftrightarrow R(u)=\frac{P_{+}+u}{P_{+}-u}=\frac{N \widehat{C}_{T}+\frac{1+N}{2 N}+u}{N \widehat{C}_{T}+\frac{1+N}{2 N}-u},
$$

where $u$ is the spectral parameter. Solution (3.1.16) is unitary $P R(u) P R(-u)=$ $R(u) R(-u)=1$ and is defined up to multiplication by an arbitrary function $f(u)$ that satisfies $f(u) f(-u)=1$.

\subsubsection{Operator $\widehat{C}$ for $s \ell(N)$ in the adjoint representation.}

In this paper we use the standard index-free matrix notation. Namely, let $A$ be an operator in $V_{N} \otimes V_{N}$, where $V_{N}=\mathbb{C}^{N}$ is the space of the defining representation of the algebra $s \ell(N)$. The operator $A$ is defined by the relations

$$
A\left(e_{k} \otimes e_{l}\right)=e_{i} \otimes e_{j} A_{k l}^{i j}
$$


where $A_{k l}^{i j}$ are the components of $A$ in the basis $\left\{e_{i} \otimes e_{j}\right\}_{i, j=1}^{N} \in V_{N}^{\otimes 2}$. Then, $A_{a b}$ denotes the action of the operator $A$ in the space $V_{N}^{\otimes 4}$ so that it is nontrivial only in the $a$-th and $b$-th factors in the product $V_{N} \otimes V_{N} \otimes V_{N} \otimes V_{N}$ (cf. (2.1.9) $)$. For example, the operator $A_{13}$ has the components

$$
\left(A_{13}\right)_{j_{1} j_{2} j_{3} j_{4}}^{i_{1} i_{2} i_{3} i_{4}}=A^{i_{1} i_{3}}{ }_{j_{1} j_{3}} \delta_{j_{2}}^{i_{2}} \delta_{j_{4}}^{i_{4}}
$$

in the basis $e_{i_{1}} \otimes e_{i_{2}} \otimes e_{i_{3}} \otimes e_{i_{4}} \in V_{N}^{\otimes 4}$ and so on. In addition to the permutation operator $P$, which is defined in (3.1.10), we need one more operator $K$ acting in $V_{N}^{\otimes 2}$ :

$$
K \cdot\left(e_{m} \otimes e_{n}\right)=\left(e_{i} \otimes e_{j}\right) \delta^{i j} \delta_{m n} \Rightarrow K_{m n}^{i j}=\delta^{i j} \delta_{m n}
$$

The operators $P_{a b}$ and $K_{a b}$ acting in the space $V_{N}^{\otimes 4}$ satisfy the following useful relations:

$$
\begin{gathered}
K_{a b}=K_{b a}, \quad K_{a b}^{2}=N K_{a b}, \quad P_{a b} K_{a b}=K_{a b}, \quad K_{a b} K_{b c}=K_{a b} P_{a c}=P_{a c} K_{b c} \\
K_{a b} K_{b c} K_{a b}=K_{a b}=K_{a b} P_{b c} K_{a b}, \quad P_{a b} K_{\mathrm{ad}} K_{b c}=P_{c d} K_{\mathrm{ad}} K_{b c}
\end{gathered}
$$

which are specific to matrix representations of the Brauer algebra generators (see e.g. [8], [18]).

The split Casimir operator $\widehat{C}_{\text {ad }}$ for the algebra $s \ell(N)$ in the adjoint representation is given by formula (2.2.1) and acts in $V_{\text {ad }} \otimes V_{\text {ad }} \subset V_{N}^{\otimes 4}$. Taking into account the definitions (3.1.3) and (3.1.7), we have

$$
\begin{gathered}
\left(\widehat{C}_{\mathrm{ad}}\right)_{j_{1} j_{2} j_{3} j_{4}}^{i_{1} i_{2} i_{3} i_{4}}=\mathrm{g}^{k_{1} k_{2}, k_{3} k_{4}} \operatorname{ad}\left(T_{k_{1} k_{2}}\right)_{j_{1} j_{2}}^{i_{1} i_{2}} \operatorname{ad}\left(T_{k_{3} k_{4}}\right)_{j_{3} j_{4}}^{i_{3} i_{4}}= \\
=\mathrm{g}^{k_{1} k_{2}, k_{3} k_{4}} C_{k_{1} k_{2}, j_{1} j_{2}}^{i_{1} i_{2}} C_{k_{3} k_{4}, j_{3} j_{4}}^{i_{3} i_{4}}=\frac{1}{2 N}\left(P_{13}+P_{24}-K_{14}-K_{23}\right)_{j_{1} j_{2} j_{3} j_{4}}^{i_{1} i_{2} i_{3} i_{4}} .
\end{gathered}
$$

Note that here the operators $\operatorname{ad}\left(T_{i k}\right)$ act in the adjoint representation space $V_{\text {ad }}$, which we identify with the space of the second rank traceless tensors $V_{\text {ad }} \equiv \bar{I} \cdot V_{N}^{\otimes 2}=\left(I-\frac{1}{N} K\right) \cdot V_{N}^{\otimes 2}$, where the projector $\bar{I}$ was introduced in (3.1.6). It means that the indices of the adjoint representation are associated in formula (3.1.19) with the pairs of indices $\left(i_{1} i_{2}\right),\left(j_{3} j_{4}\right)$ etc., possessing the traceless property, i.e. the contraction of the indices in each such pair gives zero. Setting in (3.1.19) $j_{1}=i_{3}$ and $j_{2}=i_{4}$ and summing over $i_{3}$ and $i_{4}$, we obtain the value of the quadratic Casimir operator (2.1.4) of the algebra $s \ell(N)$ in the adjoint representation

$$
\operatorname{ad}\left(C_{(2)}\right)_{12}=\operatorname{Tr}_{34}\left(\widehat{C}_{\mathrm{ad}}\right)=\frac{1}{2 N} \operatorname{Tr}_{34}\left(\left(P_{13}+P_{24}-K_{14}-K_{23}\right) P_{13} P_{24}\right)=\bar{I}_{12}
$$

i.e. $c_{(2)}^{\text {ad }}=1$, which is consistent with the general formula (2.2.9) .

Next, we need three more operators $\mathbf{K}, \mathbf{P}^{(a d)}$ and $\mathbf{P}$, where the first two act in $V_{\mathrm{ad}}^{\otimes 2}$, and the last one acts in $V_{N}^{\otimes 4}$. The operator $\mathbf{K}$ is defined as follows (cf. (3.1.17)):

$$
\begin{gathered}
\mathbf{K}_{j_{1} j_{2} j_{3} j_{4}}^{i_{1} i_{2} i_{i}}=\mathrm{g}^{i_{1} i_{2} i_{3} i_{4}} \mathrm{~g}_{j_{1} j_{2} j_{3} j_{4}}=\left(\delta^{i_{2} i_{3}} \delta^{i_{1} i_{4}}-\frac{1}{N} \delta^{i_{1} i_{2}} \delta^{i_{3} i_{4}}\right)\left(\delta_{j_{2} j_{3}} \delta_{j_{1} j_{4}}-\frac{1}{N} \delta_{j_{1} j_{2}} \delta_{j_{3} j_{4}}\right)= \\
=\left(K_{23} K_{14}-\frac{1}{N} P_{24} K_{12} K_{34}-\frac{1}{N} P_{13} K_{23} K_{14}+\frac{1}{N^{2}} K_{12} K_{34}\right)_{j_{1} j_{2} j_{3} j_{4}}^{i_{1} i_{2} i_{3} i_{4}} .
\end{gathered}
$$

The operator $\mathbf{P}$ permutes in the tensor product $V_{N}^{\otimes 4}$ the first factor with the third one and the second factor with the fourth one and has an explicit form

$$
\mathbf{P}_{j_{1} j_{2} j_{3} j_{4}}^{i_{1} i_{2} i_{3} i_{4}}=\delta_{j_{3}}^{i_{1}} \delta_{j_{1}}^{i_{3}} \delta_{j_{4}}^{i_{2}} \delta_{j_{2}}^{i_{4}} \Rightarrow \mathbf{P}=P_{13} P_{24} \quad \Rightarrow \quad \mathbf{P}^{2}=I
$$


where $I$ is the unit operator in $V_{N}^{\otimes 4}$. Finally, the operator

$$
\mathbf{P}^{(a d)} \equiv \bar{I}_{12} \bar{I}_{34} \mathbf{P}
$$

plays the role of the permutation operator in the space $V_{\text {ad }} \otimes V_{\text {ad }} \subset V_{N}^{\otimes 4}$. We stress that $\mathbf{P}$ commutes with both $\widehat{C}_{\text {ad }}$ and $\mathbf{K}$

$$
\mathbf{P} \widehat{C}_{\mathrm{ad}}=\widehat{C}_{\mathrm{ad}} \mathbf{P}, \quad \mathbf{P} \mathbf{K}=\mathbf{K}=\mathbf{K} \mathbf{P},
$$

and therefore $\mathbf{P}$ can be diagonalized simultaneously with $\widehat{C}_{\text {ad }}$ and $\mathbf{K}$.

We also note that one cannot choose in the definition of the permutation (3.1.22) in $V_{\text {ad }}^{\otimes 2}$ instead of $\mathbf{P}=P_{13} P_{24}$ another operator $\mathbf{P}^{\prime}=P_{14} P_{23}$. This is because actually $V_{\text {ad }}=\bar{I}_{12} \cdot\left(V_{N} \otimes \bar{V}_{N}\right)$, where $\bar{V}_{N}$ is the space of the contragradient representation of $\operatorname{s\ell }(N)$ (see Remark after (3.1.7)), and the element $A \in S L(N)$ acts in the space $V_{\text {ad }} \otimes V_{\text {ad }}$ as follows:

$$
V_{\text {ad }} \otimes V_{\text {ad }} \rightarrow\left(A \otimes A^{-1 \mathrm{~T}} \otimes A \otimes A^{-1 \mathrm{~T}}\right) V_{\text {ad }} \otimes V_{\text {ad }},
$$

and this action commutes with $\mathbf{P}$ but does not commute with $\mathbf{P}^{\prime}$.

Using the permutations $\mathbf{P}$ and $\mathbf{P}^{(a d)}$, we define the symmetrizer $\mathbf{P}_{+}^{(a d)}$ and the antisymmetrizer $\mathbf{P}_{-}^{(a d)}$ in the space $\left(V_{\text {ad }}\right)^{\otimes 2}$ :

$$
\begin{gathered}
\mathbf{P}_{ \pm}^{(a d)} \equiv \frac{1}{2}\left(\mathbf{I} \pm \mathbf{P}^{(a d)}\right)=\frac{1}{2}(I \pm \mathbf{P}) \bar{I}_{12} \bar{I}_{34}=\frac{1}{2} \bar{I}_{12} \bar{I}_{34}(I \pm \mathbf{P}) \\
\mathbf{P}_{-}^{(a d)}=\frac{1}{2}\left(1-P_{13} P_{24}\right)\left(1-\frac{1}{N}\left(K_{12}+K_{34}\right)\right) \\
\mathbf{P}_{+}^{(a d)}=\frac{1}{2}\left(1+P_{13} P_{24}\right)\left(1-\frac{1}{N}\left(K_{12}+K_{34}\right)+\frac{1}{N^{2}} K_{12} K_{34}\right)
\end{gathered}
$$

where $\mathbf{I}=\bar{I}_{12} \bar{I}_{34}$ is the unit operator in $\left(V_{\mathrm{ad}}\right)^{\otimes 2}$ (we often write unit 1 instead of the unit operator $I$ in $\left.V_{N}^{\otimes 4}\right)$. We define, respectively, the symmetrized and antisymmetrized parts of the Casimir operator (3.1.19)

$$
\begin{gathered}
\widehat{C}_{+}=\mathbf{P}_{+}^{(a d)} \widehat{C}_{\mathrm{ad}}=\frac{1}{2}(1+\mathbf{P}) \widehat{C}_{\mathrm{ad}}=\frac{1}{4 N}\left(1+P_{13} P_{24}\right)\left(2 P_{13}-K_{14}-K_{23}\right)= \\
=\frac{1}{4 N}\left(2 P_{13}+2 P_{24}-\left(1+P_{13} P_{24}\right) K_{14}-\left(1+P_{13} P_{24}\right) K_{23}\right), \\
\widehat{C}_{-}=\mathbf{P}_{-}^{(a d)} \widehat{C}_{\mathrm{ad}}=\frac{1}{2}(I-\mathbf{P}) \widehat{C}_{\mathrm{ad}}=\frac{1}{4 N}\left(P_{13} P_{24}-1\right)\left(K_{14}+K_{23}\right),
\end{gathered}
$$

where we used the equations $\bar{I}_{12} \widehat{C}_{\text {ad }}=\widehat{C}_{\text {ad }}=\bar{I}_{34} \widehat{C}_{\text {ad }}$, which are easily checked with the help of explicit formula (3.1.19). For $\widehat{C}_{+}$and $\widehat{C}_{-}$in view of (3.1.23) we have the following relations:

$$
\widehat{C}_{+}+\widehat{C}_{-}=\widehat{C}_{\mathrm{ad}}, \quad \mathbf{P} \widehat{C}_{ \pm}=\widehat{C}_{ \pm} \mathbf{P}, \quad \widehat{C}_{+} \widehat{C}_{-}=0=\widehat{C}_{-} \widehat{C}_{+} .
$$

In addition, we have

$$
\begin{gathered}
\mathbf{K} \widehat{C}_{-}=0=\widehat{C}_{-} \mathbf{K}, \quad \mathbf{K} \widehat{C}_{+}=-\mathbf{K}=\widehat{C}_{+} \mathbf{K}, \\
\mathbf{K} \widehat{C}_{\mathrm{ad}}=-\mathbf{K}=\widehat{C}_{\mathrm{ad}} \mathbf{K},
\end{gathered}
$$

which are nothing but formulas (2.2.6) valid for all simple Lie algebras. The second chain of equalities in (3.1.29) is derived by means of relations (3.1.18). 
Proposition 3.1.1 The antisymmetrized $\widehat{C}_{-}$and symmetrized $\widehat{C}_{+}$parts of the split Casimir operator of the Lie algebra $s \ell(N)$ (defined in (3.1.27) and (3.1.26)) satisfy the identities

$$
\begin{gathered}
\widehat{C}_{-}^{2}+\frac{1}{2} \widehat{C}_{-}=0 \Rightarrow \widehat{C}_{-}\left(\widehat{C}_{-}+\frac{1}{2}\right)=0 . \\
\widehat{C}_{+}^{3}+\frac{1}{2} \widehat{C}_{+}^{2}-\frac{1}{N^{2}} \widehat{C}_{+}-\frac{1}{4 N^{2}}\left(\mathbf{I}^{(a d)}+\mathbf{P}^{(a d)}-2 \mathbf{K}\right)=0, \\
\widehat{C}_{+}\left(\widehat{C}_{+}+\frac{1}{2}\right)\left(\widehat{C}_{+}-\frac{1}{N}\right)\left(\widehat{C}_{+}+\frac{1}{N}\right)=\frac{1}{2 N^{2}} \mathbf{K}, \\
\left(\widehat{C}_{+}+1\right)\left(\widehat{C}_{+}+\frac{1}{2}\right)\left(\widehat{C}_{+}-\frac{1}{N}\right)\left(\widehat{C}_{+}+\frac{1}{N}\right) \mathbf{P}_{+}^{(a d)}=0, \\
\widehat{C}_{+}\left(\widehat{C}_{+}+1\right)\left(\widehat{C}_{+}+\frac{1}{2}\right)\left(\widehat{C}_{+}-\frac{1}{N}\right)\left(\widehat{C}_{+}+\frac{1}{N}\right)=0,
\end{gathered}
$$

The split Casimir operator $\widehat{C}_{\mathrm{ad}}=\widehat{C}_{+}+\widehat{C}_{-}$satisfies the characteristic identity (cf. (2.3.5))

$$
\widehat{C}_{\text {ad }}\left(\widehat{C}_{\text {ad }}+\frac{1}{2}\right)\left(\widehat{C}_{\mathrm{ad}}+1\right)\left(\widehat{C}_{\mathrm{ad}}-\frac{1}{N}\right)\left(\widehat{C}_{\mathrm{ad}}+\frac{1}{N}\right)=0 .
$$

Proof. Identity (3.1.30) follows from the general statement (2.2.5), which is valid for all simple Lie algebras. Further, the identities (3.1.31) - (3.1.34) can be proved by using the diagram technique developed in [4. Nevertheless, we give here a direct algebraic proof of these identities that uses relations (3.1.18) arising in the matrix representations of the Brauer algebra (see e.g. [8], [18]). First, we calculate

$$
\begin{gathered}
\widehat{C}_{+}^{2}=\frac{1}{4}(\mathbf{I}+\mathbf{P}) \widehat{C}_{\mathrm{ad}}(\mathbf{I}+\mathbf{P}) \widehat{C}_{\mathrm{ad}}=\frac{1}{2}(\mathbf{I}+\mathbf{P}) \widehat{C}_{\mathrm{ad}}^{2}= \\
=\frac{1}{8 N^{2}}\left(1+P_{13} P_{24}\right)\left(4-2 P_{13}\left(K_{12,34}+K_{14,23}\right)+N K_{14,23}+2 K_{14} K_{23}\right),
\end{gathered}
$$

where we introduce the notation $K_{i j, k \ell} \equiv K_{i j}+K_{k \ell}$. Multiply the left and right sides of (3.1.36) by $\widehat{C}_{+}$. As a result, we get

$$
\begin{gathered}
\widehat{C}_{+}^{3}=\frac{1}{16 N^{3}}(1+\mathbf{P})\left(8 P_{13}-4\left(K_{12,34}+K_{14,23}\right)+\right. \\
\left.+2 N P_{13}\left(K_{12,34}+K_{14,23}\right)+4 P_{13}\left(K_{12} K_{34}+K_{14} K_{23}\right)-N^{2} K_{14,23}-6 N K_{14} K_{23}\right)= \\
=-\frac{1}{2} \widehat{C}_{+}^{2}+\frac{1}{N^{2}} \widehat{C}_{+}+\frac{1}{4 N^{3}}(1+\mathbf{P})\left(N-K_{12,34}+P_{13}\left(K_{14} K_{23}+K_{12} K_{34}\right)-N K_{14} K_{23}\right)= \\
=-\frac{1}{2} \widehat{C}_{+}^{2}+\frac{1}{N^{2}} \widehat{C}_{+}+\frac{1}{4 N^{2}}\left(\mathbf{I}^{(a d)}+\mathbf{P}^{(a d)}\right)-\frac{1}{2 N^{2}} \mathbf{K},
\end{gathered}
$$

where in the last equality we used formulas (3.1.20), (3.1.25) and

$$
\left(N-K_{12,34}\right)=\left(N \bar{I}_{12} \bar{I}_{34}-\frac{1}{N} K_{12} K_{34}\right), \quad \bar{I} \equiv I-\frac{1}{N} K, \quad \mathbf{P} \equiv P_{13} P_{24} .
$$

Thus, identity (3.1.31) is proved. Again, we multiply both sides of equality (3.1.31) by $\widehat{C}_{+}$and obtain

$$
\widehat{C}_{+}^{4}+\frac{1}{2} \widehat{C}_{+}^{3}-\frac{1}{N^{2}} \widehat{C}_{+}^{2}-\frac{1}{2 N^{2}} \widehat{C}_{+}=\frac{1}{2 N^{2}} \mathbf{K}
$$

that is equivalent to (3.1.32). Substitution of the expression (3.1.37) of the operator $\mathbf{K}$ into relation (3.1.31) gives (3.1.33). Identity (3.1.34) is obtained either by multiplying 
(3.1.33) by $\widehat{C}_{+}$, or by multiplying both sides of equality (3.1.32) by $\left(\widehat{C}_{+}+1\right)$ and taking into account relation $\mathbf{K}\left(\widehat{C}_{+}+1\right)=0$, which follows from (3.1.29).

Note that the antisymmetric part $\widehat{C}_{-}$of the Casimir operator, in view of the relation (3.1.30), satisfies the same relation (3.1.34) as the symmetric part of $\widehat{C}_{+}$. Hence, taking into account the last relation in (3.1.28), it follows that the complete Casimir operator $\widehat{C}_{\text {ad }}=\widehat{C}_{+}+\widehat{C}_{-}$for the algebra $s \ell(N)$ will obey a characteristic identity (33.1.35) similar to (3.1.34).

Now we show that in the $s \ell(N)$ case, in addition to the invariant operators in $V_{\mathrm{ad}}^{\otimes 2}$ represented as polynomials in $\widehat{C}_{\text {ad }}, \widehat{C}_{ \pm}$, there is one more invariant operator $Q_{-}$in $V_{\text {ad }}^{\otimes 2}$, which commutes with $\widehat{C}_{\text {ad }}, \widehat{C}_{ \pm}$but is not expressed as a polynomial in $\widehat{C}_{\text {ad }}, \widehat{C}_{ \pm}$. To construct such an operator, note that the generators $T_{a}=T\left(X_{a}\right)$ of $\operatorname{s\ell }(N)$ in the defining representation $T$ together with the unit matrix $I_{N}$ form a basis in the space of all $N \times N$ matrices; therefore, along with the defining relation (2.1.1), there is one more relation

$$
\left[T_{a}, T_{b}\right]_{+}=D_{a b}^{c} T_{c}+\alpha I_{N} \mathrm{~g}_{a b}, \quad \alpha \equiv \frac{2 c_{2}(T)}{N}
$$

Here the parameter $\alpha$ is fixed by the condition (2.3.1), and $D_{a b}^{c}$ are new structure constants of the algebra $s \ell(N)$, symmetric with respect to permutation of subscript indices $a$ and b. Now we define the operators $Q_{\text {and }} Q_{-}$in the space $V_{\mathrm{ad}}^{\otimes 2}($ cf. (2.2.1) and (2.2.4) $)$

$$
\mathrm{Q}_{b_{1} b_{2}}^{a_{1} a_{2}} \equiv \mathrm{g}^{d f} C_{d b_{1}}^{a_{1}} D_{f b_{2}}^{a_{2}}, \quad \mathbf{Q}_{-} \equiv \frac{N}{4}(\mathbf{I}-\mathbf{P}) \mathrm{Q}(\mathbf{I}-\mathbf{P})
$$

where $\mathbf{I}$ and $\mathbf{P}$ are the unit matrix and the permutation matrix in $V_{\mathrm{ad}}^{\otimes 2}$, respectively. Note that operator $\mathbf{Q}_{-}$acts nontrivially in the antisymmetric part $\mathbf{P}_{-}^{(a d)} V_{\mathrm{ad}}^{\otimes 2}$ of the space $V_{\mathrm{ad}}^{\otimes 2}$.

Proposition 3.1.2 The operator $Q_{-}$given in (3.1.39) is written as an operator in the space $V_{\mathrm{ad}}^{\otimes 2}=\bar{I}_{12} \bar{I}_{34}\left(V_{N}^{\otimes 4}\right)$ as follows:

$$
\left(\mathrm{Q}_{-}\right)_{1234}=\frac{1}{2}\left(P_{13}-P_{24}\right)\left(1-\frac{1}{N}\left(K_{12}+K_{14}+K_{23}+K_{34}\right)\right) \text {. }
$$

The operator $\mathrm{Q}_{-}$satisfies the relations

$$
\begin{gathered}
\mathbf{P}_{+}^{(a d)} \mathbf{Q}_{-}=0=\mathrm{Q}_{-} \mathbf{P}_{+}^{(a d)}, \quad \mathrm{Q}_{-} \widehat{C}_{-}=\widehat{C}_{-} \mathrm{Q}_{-}=0, \quad \mathrm{Q}_{-}^{2}=2 \widehat{C}_{-}+\mathbf{P}_{-}^{(a d)}, \\
\mathrm{Q}_{-}\left(\mathrm{Q}_{-}+1\right)\left(\mathrm{Q}_{-}-1\right)=0,
\end{gathered}
$$

where $\widehat{C}_{-}$and $\mathbf{P}_{ \pm}^{(a d)}$ are given in (3.1.27) and (3.1.25).

Proof. Relations (3.1.38) in the basis (3.1.1) are represented as

$$
\left[T_{i j}, T_{k m}\right]_{+}=D_{i j, k m}^{r s} T_{r s}+\frac{1}{N^{2}} \mathrm{~g}_{i j, k m} I_{N}
$$

where for the structure constants we have the explicit expressions

$$
D_{i j, k m}^{r s} \equiv\left(\delta_{\ell}^{r} \delta_{n}^{s}-\frac{1}{N} K_{\ell n}^{r s}\right) \bar{D}_{i j, k m}^{\ell n}, \quad \bar{D}_{i j, k m}^{r s} \equiv \delta_{j k} \delta_{i}^{r} \delta_{m}^{s}+\delta_{i m} \delta_{k}^{r} \delta_{j}^{s}-\frac{2}{N}\left(\delta_{i j} \delta_{k}^{r} \delta_{m}^{s}+\delta_{k m} \delta_{i}^{r} \delta_{j}^{s}\right) .
$$


Then the operators $\mathrm{Q}$ and $\mathrm{Q}_{-}$, given in (3.1.39), are equal to

$$
\begin{gathered}
\mathrm{Q}_{j_{1} j_{2} j_{3} j_{4}}^{i_{1} i_{2} i_{i} i_{4}}=\mathrm{g}^{k_{1} k_{2}, k_{3} k_{4}} C_{k_{1} k_{2}, j_{1} j_{2}}^{i_{1} i_{2}} D_{k_{3} k_{4}, j_{3} j_{4}}^{i_{3} i_{4}}= \\
=\frac{1}{2 N}\left(\bar{I}_{34}\left(P_{13}-P_{24}+K_{14}-K_{23}+\frac{2}{N}\left(P_{24}-P_{13}\right) K_{34}\right)\right)_{j_{1} j_{2} j_{3} j_{4}}^{i_{1} i_{2} i_{3} i_{4}}, \\
\mathbf{Q}_{-}=\frac{N}{4}(1-\mathbf{P}) \bar{I}_{12} \bar{I}_{34} \mathbf{Q} \bar{I}_{12} \bar{I}_{34}(1-\mathbf{P})= \\
=\frac{1}{8}(1-\mathbf{P})\left(P_{13}-P_{24}-\frac{2}{N} K_{34}\left(P_{13}-P_{24}\right)-\frac{2}{N}\left(P_{13}-P_{24}\right) K_{34}\right)(1-\mathbf{P}),
\end{gathered}
$$

and the right-hand side of (3.1.44) after the substitution $\mathbf{P}=P_{13} P_{24}$ matches the righthand side of (3.1.40). Relations (3.1.41) and (3.1.42) are verified by direct calculations.

Remark. Characteristic identity (3.1.42) for the operator Q $_{-}$allows us to build three mutually orthogonal projectors:

$$
\begin{gathered}
\widetilde{\mathrm{P}}_{0}^{(-)}=-\left(\mathrm{Q}_{-}+1\right)\left(\mathrm{Q}_{-}-1\right) \mathbf{P}_{-}^{(a d)}=-2 \widehat{C}_{-} \\
\widetilde{\mathrm{P}}_{ \pm 1}^{(-)}=\frac{1}{2} \mathrm{Q}_{-}\left(\mathrm{Q}_{-} \pm 1\right)=\widehat{C}_{-}+\frac{1}{2} \mathbf{P}_{-}^{(a d)} \pm \frac{1}{2} \mathrm{Q}_{-}
\end{gathered}
$$

Due to the relation $\mathbf{P}_{-}^{(a d)}=\widetilde{\mathrm{P}}_{+1}^{(-)}+\widetilde{\mathrm{P}}_{-1}^{(-)}+\widetilde{\mathrm{P}}_{0}^{(-)}$, the projectors $\widetilde{\mathrm{P}}_{0}^{(-)}, \widetilde{\mathrm{P}}_{+1}^{(-)}, \widetilde{\mathrm{P}}_{-1}^{(-)}$decompose the space $\mathbf{P}_{-}^{(a d)}\left(V_{\mathrm{ad}} \otimes V_{\mathrm{ad}}\right)$ of the antisymmetric part $\mathbb{A}(\mathrm{ad} \otimes \mathrm{ad})$ of the representation $(\mathrm{ad})^{\otimes 2}$ into eigenspaces of the operator $Q_{-}$with eigenvalues $0,+1,-1$ (which are the roots of the equation (3.1.42) ).

The characteristic identity (3.1.35) also allows one to construct projectors (invariant with respect to the action (3.1.24) $)$ onto eigen-subspaces of the operator $\widehat{C}_{\text {ad }}$ in $\left(V_{\text {ad }} \otimes V_{\text {ad }}\right)$ by means of the standard methods (see Section 3.5 in [4] and Section 4.6.4 in [8]):

$$
\mathrm{P}_{\left(a_{j}\right)}=\prod_{\substack{i=1 \\ i \neq j}}^{5} \frac{\widehat{C}_{\mathrm{ad}}-a_{i} \mathbf{I}}{a_{j}-a_{i}}
$$

where $a_{i}$ are the roots of the characteristic equation (3.1.35)

$$
a_{1}=0, \quad a_{2}=-1 / 2, \quad a_{3}=-1, \quad a_{4}=1 / N, \quad a_{5}=-1 / N
$$

Note that the case $N=2$ is special since in this case we have $\left(a_{2}-a_{5}\right)=0$, and projectors $\mathrm{P}_{\left(a_{2}\right)}=\mathrm{P}_{(-1 / 2)}$ and $\mathrm{P}_{\left(a_{5}\right)}=\mathrm{P}_{(-1 / N)}$ are not defined (see below (3.1.47)). In addition, we note that in general the projectors (3.1.46) are not primitive and extract invariant subspaces in $\left(V_{\text {ad }}\right)^{\otimes 2} \subset V_{N}^{\otimes 4}$, which are not the spaces of the irreducible representations of $s \ell(N)$. First of all, this is due to the presence of the invariant permutation operator $\mathbf{P}$ that commutes with $\widehat{C}_{\text {ad }}$ (see (3.1.23) ) and allows us to split the projectors into two parts $\mathrm{P}_{\left(a_{j}\right)}^{( \pm)}=\mathbf{P}_{ \pm}^{(a d)} \cdot \mathrm{P}_{\left(a_{j}\right)}$, where $\mathbf{P}_{ \pm}^{(a d)} \equiv \frac{1}{2}(\mathbf{I} \pm \mathbf{P}) \bar{I}_{12} \bar{I}_{34}$. From the condition (3.1.30), which can be written as $\mathbf{P}_{-}^{(a d)} \widehat{C}_{\mathrm{ad}}\left(\widehat{C}_{\mathrm{ad}}+\frac{1}{2}\right)=0$, it immediately follows that

$$
\mathrm{P}_{(-1)}^{(-)}=\mathbf{P}_{-}^{(a d)} \mathrm{P}_{(-1)}=0, \quad \mathrm{P}_{( \pm 1 / N)}^{(-)}=\mathbf{P}_{-}^{(a d)} \mathrm{P}_{( \pm 1 / N)}=0
$$

Moreover, due to relation (3.1.33) for the symmetrized part of $\mathrm{P}_{(0)}$ we obtain

$$
\mathrm{P}_{(0)}^{(+)}=\frac{\left(\widehat{C}_{\mathrm{ad}}+1\right)\left(\widehat{C}_{\mathrm{ad}}+\frac{1}{2}\right)\left(\widehat{C}_{\mathrm{ad}}+\frac{1}{N}\right)\left(\widehat{C}_{\mathrm{ad}}-\frac{1}{N}\right)}{(+1)\left(+\frac{1}{2}\right)\left(\frac{1}{N}\right)\left(-\frac{1}{N}\right)} \mathbf{P}_{+}^{(a d)}=0
$$


while the antisymmetrized part

$$
\mathrm{P}_{(0)}^{(-)}=\frac{\left(\widehat{C}_{\mathrm{ad}}+1\right)\left(\widehat{C}_{\mathrm{ad}}+\frac{1}{2}\right)\left(\widehat{C}_{\mathrm{ad}}+\frac{1}{N}\right)\left(\widehat{C}_{\mathrm{ad}}-\frac{1}{N}\right)}{(+1)\left(+\frac{1}{2}\right)\left(\frac{1}{N}\right)\left(-\frac{1}{N}\right)} \mathbf{P}_{-}^{(a d)}=2 \widehat{C}_{-}+\mathbf{P}_{-}^{(a d)} \equiv \widetilde{\mathrm{P}}_{(+1)}^{(-)}+\widetilde{\mathrm{P}}_{(-1)}^{(-)},
$$

is not primitive since it is equal to the sum of the projectors $\widetilde{\mathrm{P}}_{( \pm 1)}^{(-)}$from (3.1.45).

As a result, for $N>3$ we have 7 nontrivial projectors

$$
\widetilde{\mathrm{P}}_{(+1)}^{(-)}, \quad \widetilde{\mathrm{P}}_{(-1)}^{(-)}, \quad \mathrm{P}_{\left(-\frac{1}{2}\right)}^{(-)}, \quad \mathrm{P}_{\left(-\frac{1}{2}\right)}^{(+)}, \quad \mathrm{P}_{(-1)}^{(+)}=\mathrm{P}_{(-1)}, \quad \mathrm{P}_{\left(-\frac{1}{N}\right)}=\mathrm{P}_{\left(-\frac{1}{N}\right)}^{(+)}, \quad \mathrm{P}_{\left(+\frac{1}{N}\right)}=\mathrm{P}_{\left(+\frac{1}{N}\right)}^{(+)},
$$

which extract invariant subspaces in $\left(V_{\text {ad }}\right)^{\otimes 2}$ and by construction form a complete and mutually orthogonal system. Due to (3.1.45) and (3.1.46), these projectors have the form (cf. projectors in [4], Section 9.12)

$$
\begin{array}{rlrl}
\widetilde{\mathrm{P}}_{(+1)}^{(-)} & =\widehat{C}_{-}+\frac{1}{2} \mathbf{P}_{-}^{(a d)}+\frac{1}{2} \mathrm{Q}_{-}, & \operatorname{dim}=\frac{\left(N^{2}-1\right)\left(N^{2}-4\right)}{4}, \\
\widetilde{\mathrm{P}}_{(-1)}^{(-)}=\widehat{C}_{-}+\frac{1}{2} \mathbf{P}_{-}^{(a d)}-\frac{1}{2} \mathrm{Q}_{-}, & \operatorname{dim}=\frac{\left(N^{2}-1\right)\left(N^{2}-4\right)}{4}, \\
\mathrm{P}_{\left(-\frac{1}{2}\right)}^{(-)}=\frac{\widehat{C}_{\mathrm{ad}}\left(\widehat{C}_{\mathrm{ad}}+1\right)\left(\widehat{C}_{\mathrm{ad}}+\frac{1}{N}\right)\left(\widehat{C}_{\mathrm{ad}}-\frac{1}{N}\right)}{\left(-\frac{1}{2}\right)\left(\frac{1}{2}\right)\left(-\frac{1}{2}+\frac{1}{N}\right)\left(-\frac{1}{2}-\frac{1}{N}\right)} \mathbf{P}_{-}=-2 \widehat{C}_{-} \equiv \widehat{\mathrm{P}}_{(0)}^{(-)}, & \operatorname{dim}=N^{2}-1, \\
\mathrm{P}_{\left(-\frac{1}{2}\right)}^{(+)}=\frac{\widehat{C}_{\mathrm{ad}}\left(\widehat{C}_{\mathrm{ad}}+1\right)\left(\widehat{C}_{\mathrm{ad}}+\frac{1}{N}\right)\left(\widehat{C}_{\mathrm{ad}}-\frac{1}{N}\right)}{\left(-\frac{1}{2}\right)\left(\frac{1}{2}\right)\left(-\frac{1}{2}+\frac{1}{N}\right)\left(-\frac{1}{2}-\frac{1}{N}\right)} \mathbf{P}_{+}=\frac{4}{N^{2}-4}\left(N^{2} \widehat{C}_{+}^{2}-\mathbf{P}_{+}^{(a d)}-\mathbf{K}\right), & \operatorname{dim}=N^{2}-1, \\
\mathrm{P}_{(-1)}^{(+)}=\frac{\widehat{C}_{\mathrm{ad}}\left(\widehat{C}_{\mathrm{ad}}+\frac{1}{2}\right)\left(\widehat{C}_{\mathrm{ad}}+\frac{1}{N}\right)\left(\widehat{C}_{\mathrm{ad}}-\frac{1}{N}\right)}{(-1)\left(-\frac{1}{2}\right)\left(-1+\frac{1}{N}\right)\left(-1-\frac{1}{N}\right)} \mathbf{P}_{+}=\frac{1}{\left(N^{2}-1\right)} \mathbf{K}, & \operatorname{dim}=1, \\
\mathrm{P}_{\left(\frac{1}{N}\right)}^{(+)}=\frac{\widehat{C}_{\mathrm{ad}}\left(\widehat{C}_{\mathrm{ad}}+1\right)\left(\widehat{C}_{\mathrm{ad}}+\frac{1}{2}\right)\left(\widehat{C}_{\mathrm{ad}}+\frac{1}{N}\right)}{\left(\frac{1}{N}\right)\left(\frac{1}{N}+1\right)\left(\frac{1}{N}+\frac{1}{2}\right)\left(\frac{2}{N}\right)} \mathbf{P}_{+}= & \operatorname{dim}=\frac{N^{2}(N-1)(N+3)}{4}, \\
& =-\frac{N}{2(N+1)(N+2)} \mathbf{K}+\frac{N^{2}}{(N+2)} \widehat{C}_{+}^{2}+\frac{N}{2} \widehat{C}_{+}+\frac{N}{2(N+2)} \mathbf{P}_{+}^{(a d)}, & \\
\mathrm{P}_{\left(-\frac{1}{N}\right)}^{(+)}=\frac{\widehat{C}_{\mathrm{ad}}\left(\widehat{C}_{\mathrm{ad}}+1\right)\left(\widehat{C}_{\mathrm{ad}}+\frac{1}{2}\right)\left(\widehat{C}_{\mathrm{ad}}-\frac{1}{N}\right)}{\left(-\frac{1}{N}\right)\left(-\frac{1}{N}+1\right)\left(-\frac{1}{N}+\frac{1}{2}\right)\left(-\frac{2}{N}\right)} \mathbf{P}_{+}= & \\
& =\frac{N}{2(N-1)(N-2)} \mathbf{K}-\frac{N}{(N-2)} \widehat{C}_{+}^{2}-\frac{N}{2} \widehat{C}_{+}+\frac{N}{2(N-2)} \mathbf{P}_{+}^{(a d)}, & \operatorname{dim}=\frac{N^{2}(N+1)(N-3)}{4},
\end{array}
$$

where we used the property $\widehat{C}_{\text {ad }} \mathbf{P}_{ \pm}^{(a d)}=\widehat{C}_{\text {ad }} \mathbf{P}_{ \pm}=\widehat{C}_{ \pm}$and identities (3.1.30), (3.1.31), (3.1.32), (3.1.37). Note that the projector $\mathrm{P}_{\left(-\frac{1}{2}\right)}^{(-)}$is the same as the projector $\widetilde{\mathrm{P}}_{(0)}^{(-)}$given in (3.1.45). The right column in formula (3.1.47) shows the dimensions of the invariant subspaces in $V_{\mathrm{ad}}^{\otimes 2}$, which are extracted by the corresponding projectors. The way to calculate these dimensions is shown below.

It is well known that the tensor product of two adjoint representations of the algebra $s \ell(N)$ for $N>3$ decomposes into the sum of seven irreducible representations, which can be illustrated in terms of the Young diagrams

$$
\left[2,1^{N-2}\right] \otimes\left[2,1^{N-2}\right]=[\emptyset]+\left[2^{2}, 1^{N-4}\right]+\left[3,1^{N-3}\right]+\left[3^{2}, 2^{N-3}\right]+\left[4,1^{N-2}\right]+2 \cdot\left[2,1^{N-2}\right],
$$

where the diagram $\left[2,1^{N-2}\right]$ corresponds to the adjoint representation and

$$
\begin{gathered}
\operatorname{dim}\left[2,1^{N-2}\right]=N^{2}-1, \quad \operatorname{dim}[\emptyset]=1, \quad \operatorname{dim}\left[2^{2}, 1^{N-4}\right]=\frac{N^{2}(N+1)(N-3)}{4}, \\
\operatorname{dim}\left[3,1^{N-3}\right]=\operatorname{dim}\left[3^{2}, 2^{N-3}\right]=\frac{\left(N^{2}-1\right)\left(N^{2}-4\right)}{4}, \quad \operatorname{dim}\left[4,1^{N-2}\right]=\frac{N^{2}(N-1)(N+3)}{4} .
\end{gathered}
$$


Comparing the dimensions in (3.1.47) and (3.1.48), we conclude that seven mutually orthogonal and nontrivial projectors (3.1.47), which form a complete system in the space $\left(V_{\mathrm{ad}}\right)^{\otimes 2}$, select in $\left(V_{\mathrm{ad}}\right)^{\otimes 2}$ the subspaces of all irreducible representations of $s \ell(N)$. To verify this fact, we need to calculate the dimensions of the invariant subspaces $\widetilde{V}_{\left(b_{i}\right)}^{(-)}=$ $\widetilde{\mathrm{P}}_{\left(b_{i}\right)}^{(-)}\left(V_{\mathrm{ad}} \otimes V_{\mathrm{ad}}\right)$ and $V_{\left(a_{i}\right)}^{(+)}=\mathrm{P}_{\left(a_{i}\right)}^{(+)}\left(V_{\mathrm{ad}} \otimes V_{\mathrm{ad}}\right)$, which are given in (3.1.47), and compare these dimensions with (3.1.48). A way to calculate these dimensions is to find traces of the projector (3.1.47):

$$
\operatorname{dim}\left(\widetilde{V}_{\left(b_{i}\right)}^{(-)}\right)=\operatorname{Tr}\left(\widetilde{\mathrm{P}}_{\left(b_{i}\right)}^{(-)}\right), \quad \operatorname{dim}\left(V_{\left(a_{i}\right)}^{(+)}\right)=\operatorname{Tr}\left(\mathrm{P}_{\left(a_{i}\right)}^{(+)}\right),
$$

where we introduce the notation $\operatorname{Tr} \equiv \operatorname{Tr}_{1} \operatorname{Tr}_{2} \operatorname{Tr}_{3} \operatorname{Tr}_{4}$ for the trace in $\left(V_{\mathrm{ad}}\right)^{\otimes 2} \subset\left(V_{N}\right)^{\otimes 4}$. For this calculation, we use the traces (2.2.10) of the basic operators that make up the projectors (3.1.47):

$$
\begin{gathered}
\operatorname{Tr}(\mathbf{K})=N^{2}-1, \quad \operatorname{Tr}\left(\mathbf{P}_{-}^{(a d)}\right)=\frac{1}{2}\left(N^{2}-1\right)\left(N^{2}-2\right), \quad \operatorname{Tr}\left(\mathbf{P}_{+}^{(a d)}\right)=\frac{1}{2} N^{2}\left(N^{2}-1\right), \\
\operatorname{Tr}\left(\widehat{C}_{ \pm}\right)= \pm \frac{\left(N^{2}-1\right)}{2}, \quad \operatorname{Tr}\left(\widehat{C}_{\mathrm{ad}}^{2}\right)=\left(N^{2}-1\right), \quad \operatorname{Tr}\left(\widehat{C}_{+}^{2}\right)=\frac{3}{4}\left(N^{2}-1\right), \quad \operatorname{Tr}\left(\widetilde{C}_{-}\right)=0 .
\end{gathered}
$$

Substituting these traces into expressions (3.1.49), where the projectors $\widetilde{\mathrm{P}}_{\left(b_{i}\right)}^{(-)}, \mathrm{P}_{\left(a_{i}\right)}^{(+)}$are defined in (3.1.47), we obtain the dimensions indicated in (3.1.47) that coincide with the dimensions (3.1.48).

So using the projectors $\mathbf{P}_{+}^{(a d)}$ and $\mathbf{P}_{-}^{(a d)}$, the representation (ad) ${ }^{\otimes 2}$ of the algebra $s \ell(N)$ is decomposed into the symmetric $\mathbb{S}\left(\mathrm{ad}^{\otimes 2}\right)$ and antisymmetric $\mathbb{A}\left(\mathrm{ad}^{\otimes 2}\right)$ parts. In turn, for all simple Lie algebras (see Section 2.2) the antisymmetric part $\mathbb{A}\left(\operatorname{ad}^{\otimes 2}\right)$ splits into the sum of two subrepresentations $\mathrm{X}_{1}$ and $\mathrm{X}_{2}$, which in the case of the algebra $\operatorname{s\ell }(N)$ correspond to the projectors $\mathrm{P}_{\left(-\frac{1}{2}\right)}^{(-)}$and $\mathrm{P}_{(0)}^{(-)}$and have dimensions (2.2.12):

$$
\operatorname{dim}\left(\mathbf{X}_{1}\right)=N^{2}-1=\operatorname{dim}(s \ell(N)), \quad \operatorname{dim}\left(\mathbf{X}_{2}\right)=\frac{1}{2}\left(N^{2}-1\right)\left(N^{2}-4\right) .
$$

Moreover, in the case of the Lie algebra $s \ell(N)$, the representation $\mathrm{X}_{2}$ associated with the projector $\mathrm{P}_{(0)}^{(-)}$turns out to be reducible and expands into the sum of two inequivalent irreducible representations associated with the projectors $\widetilde{\mathrm{P}}_{(+1)}^{(-)}, \widetilde{\mathrm{P}}_{(-1)}^{(-)}$and having the same dimensions: $\operatorname{dim} \widetilde{V}_{( \pm 1)}^{(-)}=\frac{1}{4}\left(N^{2}-1\right)\left(N^{2}-4\right)$.

For the Lie algebras $s \ell(N)$, when $N>3$ (the cases $N=2,3$ are special), the symmetric part $\mathbb{S}\left(\mathrm{ad}^{\otimes 2}\right)$ decomposes into the sum of four irreducible representations, one of which $\mathrm{X}_{0}$ associated with the projector $P_{(-1)}^{(+)}$is trivial and has dimension 1, and three other representations $Y_{2}, Y_{2}^{\prime}$ and $Y_{2}^{\prime \prime}$ associated with the projectors $P_{(-1 / 2)}^{(+)}, P_{(1 / N)}^{(+)}$and $P_{(-1 / N)}^{(+)}$ have the corresponding dimensions

$$
\operatorname{dim} Y_{2}=N^{2}-1, \quad \operatorname{dim} Y_{2}^{\prime}=\frac{1}{4} N^{2}(N-1)(N+3), \quad \operatorname{dim} Y_{2}^{\prime \prime}=\frac{1}{4} N^{2}(N+1)(N-3) .
$$

Note that for $N=2$ the projectors $P_{(-1 / 2)}^{(+)}$and $P_{(-1 / N)}^{(+)}$in (3.1.47) are not defined, the dimension of the representation $Y_{2}^{\prime \prime}$ becomes negative and the above expansion does not work. For $N=3$ we have $\operatorname{dim} Y_{2}^{\prime \prime}=0$ and, therefore, in the case of the algebra $s \ell(3)$, the 
representation $Y_{2}^{\prime \prime}$ dose not appear in $\mathbb{S}\left(\operatorname{ad}^{\otimes 2}\right)$, and the corresponding projector $\mathrm{P}_{-\frac{1}{N}}^{(+)}$in (3.1.47) for $N=3$ must vanish $\left.\mathrm{P}_{-\frac{1}{N}}^{(+)}\right|_{N=3}=0$, which gives

$$
\widehat{C}_{+}^{2}=-\frac{1}{6} \widehat{C}_{+}+\frac{1}{12}\left(\mathbf{I}^{(a d)}+\mathbf{P}^{(a d)}+\mathbf{K}\right)
$$

i.e. in this case, the symmetric part $\widehat{C}_{+}$of the split Casimir operator satisfies the second order identity (3.1.50), and the third order identity (3.1.31) for $N=3$ is a consequence of (3.1.50). To derive characteristic identities in the case $N=3$, we multiply both parts of (3.1.50) by $\widehat{C}_{+}$and then we multiply the resulting relation by $\left(\widehat{C}_{+}+1\right)$ and use the equality $\left(\widehat{C}_{+}+1\right) \mathbf{K}=0$. As a result, we obtain

$$
\begin{gathered}
\widehat{C}_{+}^{3}+\frac{1}{6} \widehat{C}_{+}^{2}-\frac{1}{6} \widehat{C}_{+}=-\frac{1}{12} \mathbf{K} \Rightarrow \widehat{C}_{+}\left(\widehat{C}_{+}+1\right)\left(\widehat{C}_{+}+\frac{1}{2}\right)\left(\widehat{C}_{+}-\frac{1}{3}\right)=0 \Rightarrow \\
\widehat{C}_{\text {ad }}\left(\widehat{C}_{\mathrm{ad}}+\frac{1}{2}\right)\left(\widehat{C}_{\mathrm{ad}}+1\right)\left(\widehat{C}_{\mathrm{ad}}-\frac{1}{3}\right)=0,
\end{gathered}
$$

which for the case of $s \ell(3)$ replace (3.1.32), (3.1.34) and (3.1.35).

Finally, the decomposition of the product of two adjoint representations of the algebra $s \ell(N)$ for $N>3$ is written in the form

$$
\begin{gathered}
{\left[N^{2}-1\right] \otimes\left[N^{2}-1\right]=\mathbb{A}\left(\left[N^{2}-1\right] \otimes\left[N^{2}-1\right]\right)+\mathbb{S}\left(\left[N^{2}-1\right] \otimes\left[N^{2}-1\right]\right),} \\
\mathbb{A}\left(\left[N^{2}-1\right] \otimes\left[N^{2}-1\right]\right)=\left[N^{2}-1\right] \oplus\left[\frac{\left(N^{2}-1\right)\left(N^{2}-4\right)}{4}\right] \oplus\left[\frac{\left(N^{2}-1\right)\left(N^{2}-4\right)}{4}\right], \\
\mathbb{S}\left(\left[N^{2}-1\right] \otimes\left[N^{2}-1\right]\right)=[1] \oplus\left[N^{2}-1\right] \oplus\left[\frac{N^{2}(N-1)(N+3)}{4}\right] \oplus\left[\frac{N^{2}(N+1)(N-3)}{4}\right] .
\end{gathered}
$$

This decomposition is well known and is in accordance with the general theory of the universal description of all simple Lie algebras using the Vogel parameters [11] (see also [12], [13]). We will discuss this universal description below in Section 5.

\subsection{Split Casimir operator $\widehat{C}$ for Lie algebras $s o(N)$ and $s p(2 n)$}

\subsubsection{Operator $\widehat{C}$ for $s o(N)$ and $s p(2 n)$ in the defining representation}

In this Subsection, to fix the notation, we give the well-known definition of the Lie algebras $s o(N)$ and $s p(2 n)$ which one can find in many monographs and textbooks (see e.g. [4, [5] and [7]). We prefer to give here a natural unified definition [7, 21] of these algebras since it will be useful for us below.

We introduce the metric $\left\|c_{i j}\right\|_{i, j=1, \ldots, N}$ which is equal to the unit matrix $\left\|\delta_{i j}\right\|$ in the case of the $s o(N)$ algebras and equal to the matrix

$$
\left\|c_{i j}\right\|=\left(\begin{array}{cc}
0 & I_{n} \\
-I_{n} & 0
\end{array}\right)
$$

in the case of the algebras $s p(2 n)$. Thus, we have $c_{i j}=\epsilon c_{j i}$ with $\epsilon= \pm 1$ for the $s o(n) / s p(2 n)$ cases, respectively. The inverse metric $\bar{c}^{i j}$ is defined in a standard way as $\bar{c}^{i k} c_{k j}=\delta_{j}^{i}$. We denote the space $\mathbb{C}^{N}$ of the defining representation of $s o(N)$ and $s p(N)$ as $V_{N}$. 
Using the matrix units $\left(e_{s}{ }^{t}\right)^{i}{ }_{k}=\delta_{k}^{t} \delta_{s}^{i}$, or $\left(e_{s t}\right)^{i}{ }_{k}=c_{t k} \delta_{s}^{i}$ with lowered indices, one may define the generators of $s o(N)$ and $s p(N)$ as

$$
M_{i j}=e_{i j}-\epsilon e_{j i}, \quad\left(M_{i j}\right)^{k}{ }_{l}=c_{j l} \delta_{i}^{k}-\epsilon c_{i l} \delta_{j}^{k}=2 \delta_{[i}^{k} c_{j) l}
$$

where the notation $[i j)$ means (anti-)symmetrization for the $(s o(N)-) s p(N)$ algebras. The commutation relations for both algebras acquire the generic form

$$
\left[M_{i j}, M_{k l}\right]=c_{j k} M_{i l}-\epsilon c_{i k} M_{j l}-\epsilon c_{j l} M_{i k}+c_{i l} M_{j k}=X_{i j, k l}{ }^{m n} M_{m n}
$$

with the structure constants given by

$$
X_{i j, k l}^{m n}=c_{j k} \delta_{i}^{[m} \delta_{l}^{n)}-\epsilon c_{i k} \delta_{j}^{[m} \delta_{l}^{n)}-\epsilon c_{j l} \delta_{i}^{[m} \delta_{k}^{n)}+c_{i l} \delta_{j}^{[m} \delta_{k}^{n)}=4 \delta_{[i}^{[m} c_{j][k} \delta_{l)}^{n)} .
$$

In this basis the Cartan-Killing metric reads

$$
\mathrm{g}_{i_{1} i_{2}, j_{1} j_{2}}=2(N-2 \epsilon)\left(c_{i_{2} j_{1}} c_{j_{2} i_{1}}-\epsilon c_{i_{1} j_{1}} c_{j_{2} i_{2}}\right) \equiv(N-2 \epsilon) \operatorname{Tr}\left(M_{i_{1} i_{2}} M_{j_{1} j_{2}}\right),
$$

while the inverse metric has the form

$$
\mathrm{g}^{i_{1} i_{2}, j_{1} j_{2}}=\frac{1}{8(N-2 \epsilon)}\left(\epsilon \bar{c}^{i_{1} j_{2}} \bar{c}^{i_{2} j_{1}}-\bar{c}^{i_{1} j_{1}} \bar{c}^{i_{2} j_{2}}\right) .
$$

This inverse metric is defined by the equation $\mathrm{g}_{i j, k \ell} \mathrm{g}^{k \ell, m n}=\left(\mathcal{P}^{(\epsilon)}\right)_{i j}^{m n}$, where $\left(\mathcal{P}^{(\epsilon)}\right)_{i j}^{m n} \equiv$ $\frac{1}{2}\left(\delta_{i}^{m} \delta_{j}^{n}-\epsilon \delta_{i}^{n} \delta_{j}^{m}\right)$ is the projector on the (anti)symmetric part of $V_{N}^{\otimes 2}$.

Now it is easy to calculate the split Casimir operator $\widehat{C}$ for the algebras $s o(N)$ and $s p(N)$ in the defining representation [7]

$$
\begin{aligned}
\left(\widehat{C}_{T}\right)_{\ell_{1} \ell_{2}}^{k_{1} k_{2}} & \equiv T^{\otimes 2}(\widehat{C})_{\ell_{1} \ell_{2}}^{k_{1} k_{2}}=g^{i j, n m}\left(M_{i j}\right)_{\ell_{1}}^{k_{1}}\left(M_{n m}\right)_{\ell_{2}}^{k_{2}}= \\
& =\frac{1}{2(N-2 \epsilon)}\left(\delta_{\ell_{2}}^{k_{1}} \delta_{\ell_{1}}^{k_{2}}-\epsilon \bar{c}^{k_{1} k_{2}} c_{\ell_{1} \ell_{2}}\right),
\end{aligned}
$$

or in the index-free matrix notation we have

$$
\widehat{C}_{T}=\frac{1}{2(N-2 \epsilon)}(P-\epsilon K) .
$$

Here, $P$ is the permutation operator acting in the space $V_{N}^{\otimes 2}$ (see (3.1.10)), while the operator $K$ acting in the same space $V_{N}^{\otimes 2}$ has the following components:

$$
K_{j_{1} j_{2}}^{i_{1} i_{2}}=\bar{c}^{i_{1} i_{2}} c_{j_{1} j_{2}} .
$$

Proposition 3.2.1 The characteristic identity for the split Casimir operator (3.2.8) for the algebras $s o(N)$ and $s p(N)$ in the defining representation reads

$$
\left(\widehat{C}_{T}+\frac{\mathrm{d}_{2}}{2}\right)\left(\widehat{C}_{T}-\frac{\mathrm{d}_{2}}{2}\right)\left(\widehat{C}_{T}+\frac{\mathrm{d}_{2}}{2}(N-\epsilon)\right)=0
$$

where $\mathrm{d}_{2}=1 /(N-2 \epsilon)$. 
The proof of this Proposition is straightforward.

In accordance with equation (3.2.9), the projectors on the eigenvalues of the operator $\widehat{C}_{T}$ in $V_{N}^{\otimes 2}$ have the form [4], [8]

$$
\begin{gathered}
P_{a_{1}}=\frac{\left(\widehat{C}_{T}-a_{2}\right)\left(\widehat{C}_{T}-a_{3}\right)}{\left(a_{1}-a_{2}\right)\left(a_{1}-a_{3}\right)}=\frac{1}{2}(I+P)-\frac{(1+\epsilon)}{2(1+N-\epsilon)} K \equiv P_{+}^{(\epsilon)}, \\
P_{a_{2}}=\frac{\left(\widehat{C}_{T}-a_{1}\right)\left(\widehat{C}_{T}-a_{3}\right)}{\left(a_{2}-a_{1}\right)\left(a_{2}-a_{3}\right)}=\frac{1}{2}(I-P)-\frac{(1-\epsilon)}{2(1-N+\epsilon)} K \equiv P_{-}^{(\epsilon)}, \\
P_{a_{3}}=\frac{\left(\widehat{C}_{T}-a_{1}\right)\left(\widehat{C}_{T}-a_{2}\right)}{\left(a_{3}-a_{1}\right)\left(a_{3}-a_{2}\right)}=\frac{\epsilon}{N} K \equiv P_{0}^{(\epsilon)} .
\end{gathered}
$$

Here, $a_{1}=\frac{\mathrm{d}_{2}}{2}, a_{2}=-\frac{\mathrm{d}_{2}}{2}, a_{3}=-\frac{\mathrm{d}_{2}}{2}(N-\epsilon)$ are the roots of the characteristic equation (3.2.9), while $P_{a_{1}}$ and $P_{a_{2}}$ are, respectively, the symmetrization and antisymmetrization operator for the algebras $s o(N)(\epsilon=+1)$ and $\operatorname{sp}(N)(\epsilon=-1, N=2 r)$.

Finally note that the $s o(N)$ (or $s p(N))$-symmetric solution of the Yang-Baxter equation in the defining representation (the so-called Zamolodchikov solution) can be written as follows (see e.g. [7]):

$$
\begin{gathered}
R(u)=\frac{1}{\epsilon-u}\left(u+P-\frac{\epsilon u}{(u+N / 2-\epsilon)} K\right)= \\
=\frac{(u+1)}{(\epsilon-u)} P_{+}^{(\epsilon)}+\frac{(u-1)}{(\epsilon-u)} P_{-}^{(\epsilon)}+\frac{(N / 2-\epsilon-u)}{(N / 2-\epsilon+u)} P_{0}^{(\epsilon)}, \quad P R(u) P R(-u)=1 .
\end{gathered}
$$

It is quite intriguing that the solution (3.2.11) can be elegantly represented as a rational function of the split Casimir operator

$$
R(u)=\frac{\widehat{C}_{T}+\mathrm{d}_{2}(\epsilon / 2+u)}{\widehat{C}_{T}+\mathrm{d}_{2}(\epsilon / 2-u)},
$$

where the constant $d_{2}$ was introduced in (3.2.9) (see also (2.3.1) and (3.2.5)).

\subsubsection{Operator $\widehat{C}$ for $s o(N)$ and $s p(2 n)$ in the adjoint representation}

The split Casimir operator $\widehat{C}_{\text {ad }}$ for the algebras $s o(N)$ and $s p(2 n)$ in the adjoint representation together with the construction of the projectors on the irreducible representations in $\operatorname{ad} \otimes$ ad were considered in detail in [21]. Thus, for completeness, we will present here only a short review of the results discussed in [21].

The split Casimir operator $\widehat{C}_{\text {ad }}$ for the algebras $\mathfrak{g}=s o(N), s p(N)$ in the adjoint representation can be expressed through the split Casimir operator in the defining representation $\widehat{C}_{T}$ (3.2.7) as [21]:

$$
\left(\widehat{C}_{\mathrm{ad}}\right)_{j_{1} j_{2} j_{3} j_{4}}^{k_{1} k_{2} k_{3} k_{4}}=4 \delta_{\left[j_{2}\right.}^{\left[k_{2}\right.}\left(\widehat{C}_{T}\right)_{\left.j_{1}\right)\left[j_{3}\right.}^{\left.k_{1}\right)\left[k_{3}\right.} \delta_{\left.j_{4}\right)}^{\left.k_{4}\right)}=4\left(\mathcal{P}_{12,34}^{(\epsilon)}\left(\widehat{C}_{T}\right)_{13} \mathcal{P}_{12,34}^{(\epsilon)}\right)_{j_{1} j_{2} j_{3} j_{4}}^{k_{1} k_{2} k_{3} k_{4}},
$$

where we introduce the projectors

$$
\mathcal{P}_{12,34}^{(\epsilon)}=\mathcal{P}_{12}^{(\epsilon)} \mathcal{P}_{34}^{(\epsilon)}, \quad \mathcal{P}_{a b}^{(\epsilon)} \equiv \frac{1}{2}\left(I-\epsilon P_{a b}\right),
$$

and use the index-free matrix notation explained at the beginning of Section 3.1.2. Using the known expression (3.2.8) for $\widehat{C}_{T}$, we obtain

$$
\widehat{C}_{\text {ad }}=\frac{2}{(N-2 \epsilon)} \mathcal{P}_{12,34}^{(\epsilon)}\left(P_{13}-\epsilon K_{13}\right) \mathcal{P}_{12,34}^{(\epsilon)} .
$$


Due to the existence of accidental isomorphisms $s o(3) \simeq s \ell(2) \simeq s p(2), s o(4) \simeq$ $s \ell(2)+s \ell(2), s o(5) \simeq s p(4)$ and $s o(6) \simeq s \ell(4)$, in what follows we limit ourselves to considering the algebras $s o(N)$ with $N \geq 7$ and $\operatorname{sp}(N)$ with $N=2 n \geq 4$, only.

Let us define the following operators acting in the space $V_{\text {ad }}^{\epsilon} \otimes V_{\text {ad }}^{\epsilon} \subset V_{N}^{\otimes 4}$ :

$$
\mathbf{I} \equiv \mathcal{P}_{12,34}^{(\epsilon)}=\mathcal{P}_{12}^{(\epsilon)} \mathcal{P}_{34}^{(\epsilon)}, \quad \mathbf{P} \equiv \mathcal{P}_{12,34}^{(\epsilon)} P_{13} P_{24} \mathcal{P}_{12,34}^{(\epsilon)}, \quad \mathbf{K} \equiv \mathcal{P}_{12,34}^{(\epsilon)} K_{13} K_{24} \mathcal{P}_{12,34}^{(\epsilon)}
$$

These operators obey useful relations:

$$
\begin{gathered}
\mathbf{I}=\mathbf{I} P_{12} P_{34}=P_{12} P_{34} \mathbf{I}, \quad \mathbf{P}=P_{13} P_{24} \mathbf{I}=\mathbf{I} P_{13} P_{24}, \\
\mathbf{P}^{2}=\mathbf{I}, \quad \mathbf{K} \mathbf{P}=\mathbf{P} \mathbf{K}=\mathbf{K}, \quad \mathbf{K}^{2}=\frac{M(M-1)}{2} \mathbf{K}, \quad M \equiv \epsilon N, \\
\widehat{C}_{\mathrm{ad}} \mathbf{P}=\mathbf{P} \widehat{C}_{\mathrm{ad}}, \quad \widehat{C}_{\mathrm{ad}} \mathbf{K}=\mathbf{K} \widehat{C}_{\mathrm{ad}}=-\mathbf{K},
\end{gathered}
$$

It proved useful to define the symmetric $\widehat{C}_{+}$and antisymmetric $\widehat{C}_{-}$parts of the split Casimir operator $\widehat{C}_{\text {ad }}$ as

$$
\widehat{C}_{+}=\frac{1}{2}(\mathbf{I}+\mathbf{P}) \widehat{C}_{\mathrm{ad}}, \quad \widehat{C}_{-}=\frac{1}{2}(\mathbf{I}-\mathbf{P}) \widehat{C}_{\mathrm{ad}} .
$$

In the paper [21], the following proposition was proven.

Proposition 3.2.2 The characteristic identities for the operators $\widehat{C}_{-}, \widehat{C}_{+}$and $\widehat{C}_{\mathrm{ad}}$ for the algebras so $(N)$ and $s p(N)$ read

$$
\begin{gathered}
\widehat{C}_{-}^{2}+\frac{1}{2} \widehat{C}_{-}=0 \quad \Leftrightarrow \quad \widehat{C}_{-}\left(\widehat{C}_{-}+\frac{1}{2}\right)=0 \\
\widehat{C}_{+}^{3}=-\frac{1}{2} \widehat{C}_{+}^{2}-\frac{M-8}{2(M-2)^{2}} \widehat{C}_{+}+\frac{M-4}{2(M-2)^{3}}(\mathbf{I}+\mathbf{P}-2 \mathbf{K}), \\
\widehat{C}_{+}\left(\widehat{C}_{+}+1\right)\left(\widehat{C}_{+}-\frac{1}{(M-2)}\right)\left(\widehat{C}_{+}+\frac{2}{(M-2)}\right)\left(\widehat{C}_{+}+\frac{(M-4)}{2(M-2)}\right)=0, \\
\widehat{C}_{\mathrm{ad}}\left(\widehat{C}_{\mathrm{ad}}+\frac{1}{2}\right)\left(\widehat{C}_{\mathrm{ad}}+1\right)\left(\widehat{C}_{\mathrm{ad}}-\frac{1}{M-2}\right)\left(\widehat{C}_{\mathrm{ad}}+\frac{2}{M-2}\right)\left(\widehat{C}_{\mathrm{ad}}+\frac{M-4}{2(M-2)}\right)=0,
\end{gathered}
$$

where the parameter $M=\epsilon N$ is supposed to obey $M \geq 7, M \neq 8$ for the algebras so $(N)$, and $M \leq-4$ for the algebras $s p(N)$.

Remark 1. Identity $(\underline{3.2 .20})$ is derived from the intermediate formula [21]:

$$
\widehat{C}_{+}^{2}=\frac{1}{(M-2)^{2}}(\mathbf{I}+\mathbf{P}+\mathbf{K})-\frac{1}{(M-2)} \widehat{C}_{+}+\frac{(M-8)}{2(M-2)^{2}} P_{12,34}^{(\epsilon)} K_{13}\left(1+\epsilon P_{24}\right) P_{12,34}^{(\epsilon)}
$$

which is simplified for $M=8$ and instead of (3.2.20) we obtain the identity on $\widehat{C}_{+}$of the second order

$$
\widehat{C}_{+}^{2}=-\frac{1}{6} \widehat{C}_{+}+\frac{1}{36}(\mathbf{I}+\mathbf{P}+\mathbf{K}) .
$$

That is why the case $M=8$ was excluded from Proposition 3.2.2. 
The characteristic identity (3.2.22) can be used to construct a complete system of orthogonal projectors on the invariant subspaces in $V_{\text {ad }} \otimes V_{\text {ad }}$, which are simultaneously are eigenspaces of the operator $\widehat{C}_{\text {ad }}$. They are defined in a standard way (see e.g. [4, 8]):

$$
\mathrm{P}_{j}:=\mathrm{P}_{a_{j}}=\prod_{\substack{i=1 \\ i \neq j}}^{6} \frac{\widehat{C}_{\mathrm{ad}}-a_{i} \mathbf{I}}{a_{j}-a_{i}}
$$

where $a_{i}$ are the roots of the characteristic equation (3.2.22):

$$
a_{1}=0, a_{2}=-\frac{1}{2}, a_{3}=-1, a_{4}=\frac{1}{M-2}, a_{5}=-\frac{2}{M-2}, a_{6}=-\frac{M-4}{2(M-2)},
$$

and the characteristic identity (3.2.22) is written in the form

$$
\left(\widehat{C}_{\mathrm{ad}}-a_{1}\right)\left(\widehat{C}_{\mathrm{ad}}-a_{2}\right)\left(\widehat{C}_{\mathrm{ad}}-a_{3}\right)\left(\widehat{C}_{\mathrm{ad}}-a_{4}\right)\left(\widehat{C}_{\mathrm{ad}}-a_{5}\right)\left(\widehat{C}_{\mathrm{ad}}-a_{6}\right)=0 .
$$

There are some special cases in which some of the roots (3.2.25) coincide:

- $M=4$ - algebra $s o(4)$. In this case, $a_{5}=a_{3}=-1$ and $a_{6}=a_{1}=0$. The correct characteristic identity reads

$$
\widehat{C}_{\mathrm{ad}}\left(\widehat{C}_{\mathrm{ad}}+\frac{1}{2}\right)\left(\widehat{C}_{\mathrm{ad}}+1\right)\left(\widehat{C}_{\mathrm{ad}}-\frac{1}{2}\right)=0 .
$$

In other words, the characteristic identity contains each factor $\widehat{C}_{\text {ad }}$ and $\left(\widehat{C}_{\text {ad }}+1\right)$ only once.

- $M=6$ - algebra $s o(6)$. Now, $a_{5}=a_{2}=-\frac{1}{2}$ and, therefore, the characteristic identity is of the fifth order:

$$
\widehat{C}_{\mathrm{ad}}\left(\widehat{C}_{\mathrm{ad}}+\frac{1}{2}\right)\left(\widehat{C}_{\mathrm{ad}}+1\right)\left(\widehat{C}_{\mathrm{ad}}-\frac{1}{4}\right)\left(\widehat{C}_{\mathrm{ad}}+\frac{1}{4}\right)=0 .
$$

- $M=8$ - algebra $s o(8)$. Now $a_{5}=a_{6}=-\frac{1}{3}$ and again the characteristic identity is of the fifth order (this case will be considered in detail below):

$$
\widehat{C}_{\text {ad }}\left(\widehat{C}_{\text {ad }}+\frac{1}{2}\right)\left(\widehat{C}_{\text {ad }}+1\right)\left(\widehat{C}_{\text {ad }}-\frac{1}{6}\right)\left(\widehat{C}_{\text {ad }}+\frac{1}{3}\right)=0 .
$$

- $M=5$. In virtue of the accidental automorphism $s o(5)=s p(4)$, this case is identical to the case with $M=-4$.

Explicitly, the six projectors $\mathrm{P}_{1}, \ldots, \mathrm{P}_{6}$ in (3.2.24) were calculated in [21, 4 ]

$$
\begin{aligned}
\mathrm{P}_{1} & \equiv \mathrm{P}_{1}^{-}=\frac{1}{2}(\mathbf{I}-\mathbf{P})+2 \widehat{C}_{-}, \\
\mathrm{P}_{2} & \equiv \mathrm{P}_{2}^{-}=-2 \widehat{C}_{-}, \\
\mathrm{P}_{3} & \equiv \mathrm{P}_{3}^{+}=\frac{2 \mathbf{K}}{(M-1) M} \equiv \frac{\mathbf{K}}{\operatorname{dim} \mathfrak{g}}, \\
\mathrm{P}_{4} & \equiv \mathrm{P}_{4}^{+}=\frac{2}{3}(M-2) \widehat{C}_{+}^{2}+\frac{M}{3} \widehat{C}_{+}+\frac{(M-4)(\mathbf{I}+\mathbf{P})}{3(M-2)}-\frac{2(M-4) \mathbf{K}}{3(M-2)(M-1)}, \quad(3.2 .28) \\
\mathrm{P}_{5} & \equiv \mathrm{P}_{5}^{+}=-\frac{2(M-2)^{2}}{3(M-8)} \widehat{C}_{+}^{2}-\frac{(M-2)(M-6)}{3(M-8)} \widehat{C}_{+}+\frac{(M-4)(\mathbf{I}+\mathbf{P})}{6(M-8)}+\frac{2 \mathbf{K}}{3(M-8)} \\
\mathrm{P}_{6} & \equiv \mathrm{P}_{6}^{+}=\frac{4(M-2)}{M-8} \widehat{C}_{+}^{2}+\frac{4}{M-8} \widehat{C}_{+}-\frac{4(\mathbf{I}+\mathbf{P})}{(M-2)(M-8)}-\frac{8(M-4) \mathbf{K}}{M(M-2)(M-8)}
\end{aligned}
$$


The dimensions of the corresponding representations can be easily found by means of the trace formulas (2.2.10), and we have (see [4], Table 10.3)

$$
\begin{aligned}
& \operatorname{dim}\left(V_{a_{1}}\right)=\operatorname{Tr} \mathrm{P}_{1}=\frac{1}{8} M(M-1)(M+2)(M-3), \\
& \operatorname{dim}\left(V_{a_{2}}\right)=\operatorname{Tr} \mathrm{P}_{2}=\frac{1}{2} M(M-1), \\
& \operatorname{dim}\left(V_{a_{3}}\right)=\operatorname{Tr} \mathrm{P}_{3}=1 \\
& \operatorname{dim}\left(V_{a_{4}}\right)=\operatorname{Tr} \mathrm{P}_{4}=\frac{1}{12} M(M+1)(M+2)(M-3), \\
& \operatorname{dim}\left(V_{a_{5}}\right)=\operatorname{Tr} \mathrm{P}_{5}=\frac{1}{24} M(M-1)(M-2)(M-3), \\
& \operatorname{dim}\left(V_{a_{6}}\right)=\operatorname{Tr} \mathrm{P}_{6}=\frac{1}{2}(M-1)(M+2) .
\end{aligned}
$$

Remark 2. The characteristic identities (3.2.22) and dimensions $(3.2 .29)$ for $s o(N)$ and $s p(N)$ are related by replacement $N \rightarrow-N$. This fact manifests the duality between certain formulas in the representation theories of the algebras $s p(N)$ and $s o(N)$ (see [16], [4], [17] and references therein).

\subsubsection{The algebra $s o(8)$}

For the algebra $s o(8)$ we have (3.2.19), (3.2.23):

$$
\widehat{C}_{-}^{2}=-\frac{1}{2} \widehat{C}_{-}, \quad \widehat{C}_{+}^{2}=-\frac{1}{6} \widehat{C}_{+}+\frac{1}{36}(\mathbf{I}+\mathbf{P}+\mathbf{K})
$$

and, therefore, the characteristic identity has the fourth order (cf. (3.2.21) )

$$
\widehat{C}_{+}\left(\widehat{C}_{+}+1\right)\left(\widehat{C}_{+}-\frac{1}{6}\right)\left(\widehat{C}_{+}+\frac{1}{3}\right)=0
$$

All these imply the existence of the characteristic identity for the full split Casimir operator $\widehat{C}_{\mathrm{ad}}$ of the fifth order (3.2.27). Thus, the operator $\widehat{C}_{\mathrm{ad}}$ has the following eigenvalues:

$$
a_{1}=0, \quad a_{2}=-1 / 2, \quad a_{3}=-1, \quad a_{4}=1 / 6, \quad a_{5}=-1 / 3 .
$$

All projectors $\mathrm{P}_{k}^{\prime} \equiv \mathrm{P}_{a_{k}}^{\prime}$ on the eigenspaces of the operator $\widehat{C}_{\text {ad }}$ corresponding to the eigenvalues $a_{k}$ have been constructed in [21]. However, not all $\mathrm{P}_{k}^{\prime}$ are projectors onto irreducible representations, because of the different expansion of the tensor product $\operatorname{ad}^{\otimes 2}(s o(8))$ into the irreducible representations [4, 22, 24]:

$$
\begin{gathered}
\operatorname{ad}^{\otimes 2}(s o(8))=[28]^{2}=[1]+[28]+[35]+\left[35^{\prime}\right]+\left[35^{\prime \prime}\right]+[300]+[350] \Rightarrow \\
V_{\mathrm{ad}}^{\otimes 2}=V_{1}+V_{28}+V_{35}+V_{35^{\prime}}+V_{35^{\prime \prime}}+V_{300}+V_{350} .
\end{gathered}
$$

The projector $\mathrm{P}_{5}^{\prime}=\left.\left(\mathrm{P}_{5}+\mathrm{P}_{6}\right)\right|_{M=8}$ with the eigenvalue $(-1 / 3)$ corresponds to the space with dimension 105 (see (3.2.29) ). Therefore, $\mathrm{P}_{5}^{\prime}$ is not primitive, and it can be further split into three projectors

$$
\mathrm{P}_{5}^{\prime}=\overline{\mathrm{P}}_{0}+\overline{\mathrm{P}}_{+}+\overline{\mathrm{P}}_{-}
$$


each of which corresponds to the space with dimensions 35. Finally, for the exceptional case of the algebra $s o(8)$, the complete system of primitive projectors reads [21]:

$$
\begin{array}{ll}
\mathrm{P}_{1}^{\prime}=\left.\mathrm{P}_{1}\right|_{M=8}=\frac{1}{2}(\mathbf{I}-\mathbf{P})+2 \widehat{C}_{-}, & \operatorname{dim}=350, \\
\mathrm{P}_{2}^{\prime}=\left.\mathrm{P}_{2}\right|_{M=8}=-2 \widehat{C}_{-}, & \operatorname{dim}=28, \\
\mathrm{P}_{3}^{\prime}=\left.\mathrm{P}_{3}\right|_{M=8}=\frac{1}{28} \mathbf{K}, & \operatorname{dim}=1, \\
\mathrm{P}_{4}^{\prime}=\left.\mathrm{P}_{4}\right|_{M=8}=\frac{1}{3}(\mathbf{I}+\mathbf{P})+2 \widehat{C}_{+}+\frac{1}{21} \mathbf{K}, & \operatorname{dim}=300, \\
\overline{\mathrm{P}}_{0}=\mathrm{P}_{5}^{\prime}-A_{4}=\frac{1}{6}(\mathbf{I}+\mathbf{P})-2 \widehat{C}_{+}-\frac{1}{12} \mathbf{K}-A_{4}, & \operatorname{dim}=35, \\
\overline{\mathrm{P}}_{+}=\frac{1}{2}\left(A_{4}+E_{4}\right), & \operatorname{dim}=35, \\
\overline{\mathrm{P}}_{-}=\frac{1}{2}\left(A_{4}-E_{4}\right), & \operatorname{dim}=35 .
\end{array}
$$

Here, $A_{4}$ is the antisymmetrizer in $V_{8}^{\otimes 4}$ and $E_{4}$ is the invariant operator in $V_{8}^{\otimes 4}$ with the components $\left(E_{4}\right)^{i_{1} \ldots i_{4}} j_{1} \ldots j_{4}=(4 !)^{-1} \varepsilon^{i_{1} \ldots i_{4}} j_{1} \ldots j_{4}$, where $\varepsilon^{i_{1} \ldots i_{4}}{ }_{j_{1} \ldots j_{4}}=\varepsilon^{i_{1} \ldots i_{4} j_{1} \ldots j_{4}}$ is a fully antisymmetric rank-eight tensor $\varepsilon^{123 \ldots 8}=1$. The operators $A_{4}$ and $E_{4}$ obey the conditions

$$
A_{4}^{2}=A_{4}, \quad A_{4} E_{4}=E_{4} A_{4}=E_{4}, \quad E_{4}^{2}=A_{4},
$$

and $\overline{\mathrm{P}}_{+}$and $\overline{\mathrm{P}}_{-}$are the projectors onto the self-dual and anti-self-dual parts of $V_{8}^{\wedge 4} \equiv$ $A_{4}\left(V_{8}^{\otimes 4}\right)$. We note that the operator $E_{4}$ is independent of the operators $\widehat{C}_{ \pm}$, i.e., it cannot be expressed as a polynomial function of $\widehat{C}_{ \pm}$.

\subsection{Universal characteristic identities for operator $\widehat{C}_{+}$in the case of Lie algebras of classical series}

For the algebras of the classical series $A_{n}, B_{n}, C_{n}, D_{n}$ the characteristic identities (3.1.31) and (3.2.20) for the operator $\widehat{C}_{+}$in the adjoint representation can be written in a generic form

$$
\widehat{C}_{+}^{3}+\frac{1}{2} \widehat{C}_{+}^{2}=\mu_{1} \widehat{C}_{+}+\mu_{2}\left(\mathbf{I}^{(a d)}+\mathbf{P}^{(a d)}-2 \mathbf{K}\right),
$$

where $\mu_{1}$ and $\mu_{2}$ are the parameters we define at the moment. Multiplying both sides of equation (3.3.1) by $\mathbf{K}$ and using the relations

$$
\mathbf{K}\left(\mathbf{I}^{(a d)}+\mathbf{P}^{(a d)}\right)=2 \mathbf{K}, \quad \mathbf{K} \widehat{C}_{+}=-\mathbf{K}, \quad \mathbf{K} \cdot \mathbf{K}=\operatorname{dim} \mathfrak{g} \cdot \mathbf{K},
$$

one may express the dimension of the Lie algebra $\mathfrak{g}$ through the parameters $\mu_{1}$ and $\mu_{2}$

$$
\operatorname{dim} \mathfrak{g}=\frac{2 \mu_{2}-\mu_{1}+1 / 2}{2 \mu_{2}} .
$$

Then, we multiply both sides of (3.3.1) by $\widehat{C}_{+}\left(\widehat{C}_{+}+1\right)$ and deduce the characteristic identity for $\widehat{C}_{+}$:

$$
\widehat{C}_{+}\left(\widehat{C}_{+}+1\right)\left(\widehat{C}_{+}^{3}+\frac{1}{2} \widehat{C}_{+}^{2}-\mu_{1} \widehat{C}_{+}-2 \mu_{2}\right)=0 .
$$

which can be written in a factorized form

$$
\widehat{C}_{+}\left(\widehat{C}_{+}+1\right)\left(\widehat{C}_{+}+\frac{\alpha}{2 t}\right)\left(\widehat{C}_{+}+\frac{\beta}{2 t}\right)\left(\widehat{C}_{+}+\frac{\gamma}{2 t}\right)=0 \Leftrightarrow \prod_{i=1}^{5}\left(\widehat{C}_{+}-a_{i}\right)=0 .
$$


Here we introduce the notation for the roots of the identity (3.3.3)

$$
a_{1}=0, \quad a_{2}=-1, \quad a_{3}=-\frac{\alpha}{2 t}, \quad a_{4}=-\frac{\beta}{2 t}, \quad a_{5}=-\frac{\gamma}{2 t}, \quad t=\alpha+\beta+\gamma,
$$

and the last equation follows from the condition $\left(a_{3}+a_{4}+a_{5}\right)=-1 / 2$. The parameter $t$ normalizes the eigenvalues of the operator $\widehat{C}_{+}$. For each simple Lie algebra $\mathfrak{g}$ we choose $t^{-1}$ such that

$$
(\theta, \theta)=\frac{1}{t}
$$

where $\theta$ is the highest root of $\mathfrak{g}$. Thus, $t$ coincides with the dual Coxeter number $h^{\vee}$ of the algebra $\mathfrak{g}$. The parameters $\alpha, \beta, \gamma$ were introduced by Vogel [11]. The values of these parameters for the algebras $A_{n}, B_{n}, C_{n}, D_{n}$ are extracted from identities (3.1.34), (3.2.21), and we summarize them in Table 3.

Table 3.

\begin{tabular}{|c|c|c|c|c|}
\hline & $s \ell(n+1)$ & $s o(2 n+1)$ & $s p(2 n)$ & $s o(2 n)$ \\
\hline$t$ & $n+1$ & $2 n-1$ & $n+1$ & $2 n-2$ \\
\hline$\frac{\alpha}{2 t}$ & $-1 /(n+1)$ & $-1 /(2 n-1)$ & $-1 /(n+1)$ & $-1 /(2 n-2)$ \\
\hline$\frac{\beta}{2 t}$ & $1 /(n+1)$ & $2 /(2 n-1)$ & $1 /(2 n+2)$ & $1 /(n-1)$ \\
\hline$\frac{\gamma}{2 t}$ & $1 / 2$ & $(2 n-3) /(4 n-2)$ & $(n+2) /(2 n+2)$ & $(n-2) /(2 n-2)$ \\
\hline
\end{tabular}

Comparison of equations (3.3.3) and (3.3.4) implies that the parameters $\mu_{1}$ and $\mu_{2}$ are expressed via the Vogel parameters as

$$
\mu_{1}=-\frac{\alpha \beta+\alpha \gamma+\beta \gamma}{4 t^{2}}, \quad \mu_{2}=-\frac{\alpha \beta \gamma}{16 t^{3}}
$$

and the dimensions (3.3.2) of the simple Lie algebras acquire a remarkable universal form obtained by Deligne and Vogel [10],[11]:

$$
\operatorname{dim} \mathfrak{g}=\frac{(\alpha-2 t)(\beta-2 t)(\gamma-2 t)}{\alpha \beta \gamma} .
$$

Now by using the characteristic identity (3.3.4), one can obtain the universal form of the projectors $\mathrm{P}_{\left(a_{i}\right)}^{(+)}$on the invariant subspaces $V_{\left(a_{i}\right)}$ in the symmetrized space $\frac{1}{2}\left(\mathbf{I}^{(\mathrm{ad})}+\right.$ $\left.\mathbf{P}^{(\mathrm{ad})}\right)\left(V_{\mathrm{ad}}^{\otimes 2}\right)$ :

$$
\begin{gathered}
\mathrm{P}_{\left(-\frac{\alpha}{2 t}\right)}^{(+)}=\frac{4 t^{2}}{(\beta-\alpha)(\gamma-\alpha)}\left(\widehat{C}_{+}^{2}+\left(\frac{1}{2}-\frac{\alpha}{2 t}\right) \widehat{C}_{+}+\frac{\beta \gamma}{8 t^{2}}\left(\mathbf{I}^{(\mathrm{ad})}+\mathbf{P}^{(\mathrm{ad})}-\frac{2 \alpha}{(\alpha-2 t)} \mathbf{K}\right)\right) \equiv \mathrm{P}^{(+)}(\alpha \mid \beta, \gamma), \\
\mathrm{P}_{\left(-\frac{\beta}{2 t}\right)}^{(+)}=\mathrm{P}^{(+)}(\beta \mid \alpha, \gamma), \quad \mathrm{P}_{\left(-\frac{\gamma}{2 t}\right)}^{(+)}=\mathrm{P}^{(+)}(\gamma \mid \alpha, \beta), \quad \mathrm{P}_{(-1)}^{(+)}=\frac{1}{\operatorname{dim} \mathfrak{g}} \mathbf{K} .
\end{gathered}
$$

The irreducible representations that act in the subspaces $V_{(-1)}, V_{\left(-\frac{\alpha}{2 t}\right)}, V_{\left(-\frac{\beta}{2 t}\right)}, V_{\left(-\frac{\gamma}{2 t}\right)}$ were respectively denoted in [11] as $\mathrm{X}_{0}, Y_{2}(\alpha), Y_{2}(\beta), Y_{2}(\gamma)$; see Section [5 below. Finally, we calculate (by means of trace formulas (2.2.10) ) the universal expressions [1] for the dimensions of the invariant eigenspaces $V_{\left(a_{i}\right)}$ :

$$
\begin{aligned}
& \operatorname{dim} V_{(-1)}=\operatorname{Tr} \mathrm{P}_{(-1)}^{(+)}=1 \\
& \operatorname{dim} V_{\left(-\frac{\alpha}{2 t}\right)}=\operatorname{Tr} \mathrm{P}_{\left(-\frac{\alpha}{2 t}\right)}^{(+)}=-\frac{(3 \alpha-2 t)(\beta-2 t)(\gamma-2 t) t(\beta+t)(\gamma+t)}{\alpha^{2}(\alpha-\beta) \beta(\alpha-\gamma) \gamma}, \\
& \operatorname{dim} V_{\left(-\frac{\beta}{2 t}\right)}=\operatorname{Tr} \mathrm{P}_{\left(-\frac{\beta}{2 t}\right)}^{(+)}=-\frac{(3 \beta-2 t)(\alpha-2 t)(\gamma-2 t) t(\alpha+t)(\gamma+t)}{\beta^{2}(\beta-\alpha) \alpha(\beta-\gamma) \gamma}, \\
& \operatorname{dim} V_{\left(-\frac{\gamma}{2 t}\right)}=\operatorname{Tr} \mathrm{P}_{\left(-\frac{\gamma}{2 t}\right)}^{(+)}=-\frac{(3 \gamma-2 t)(\beta-2 t)(\alpha-2 t) t(\beta+t)(\alpha+t)}{\gamma^{2}(\gamma-\beta) \beta(\gamma-\alpha) \alpha} .
\end{aligned}
$$


Here we encounter an interesting nonlinear Diophantine problem of finding all integer $\operatorname{dim} \mathfrak{g}$ in (3.3.8) for which the parameters $\alpha, \beta, \gamma$ and $\operatorname{dim} V_{\left(a_{i}\right)}$ are integers. The partial solutions of this problem are given in Table 3. The analogous Diophantine problems were considered in [19, 20].

\section{Split Casimir operators $\widehat{C}$ for exceptional Lie alge- bras}

\subsection{Characteristic identities, projectors and $R$-matrices in the fundamental representation}

\subsubsection{Basic definitions}

Let $T$ be the minimal fundamental representation of the exceptional Lie algebras $\mathfrak{g}=\mathfrak{g}_{2}, \mathfrak{f}_{4}$, $\mathfrak{e}_{6}, \mathfrak{e}_{7}, \mathfrak{e}_{8}$, acting in the space $V$. Let us choose the basis elements $X_{a}$ of the algebras $\mathfrak{g}$ so that the Cartan-Killing metric $\mathrm{g}_{a b}$ is proportional to $\delta_{a b}$, i.e.

$$
\operatorname{Tr}\left(T_{a} T_{b}\right)=d_{2} \mathrm{~g}_{a b}=-\delta_{a b},
$$

where $T_{a} \equiv T\left(X_{a}\right)$. For this normalization the split Casimir operator in the representation $T$ reads $\hat{C}=\mathrm{g}^{a b} T_{a} \otimes T_{b}=-d_{2} T_{a} \otimes T_{a}$. Below we omit the constant parameter $d_{2}$ and use the following definition for the split Casimir operator

$$
\hat{C}_{j_{1} j_{2}}^{i_{1} i_{2}}=\frac{\mathrm{g}^{a b}}{d_{2}}\left(T_{a}\right)_{j_{1}}^{i_{1}}\left(T_{b}\right)_{j_{2}}^{i_{2}}=-\left(T_{a}\right)_{j_{1}}^{i_{1}}\left(T_{a}\right)_{j_{2}}^{i_{2}}
$$

In what follows we also need the identity $I$ and permutation $P$ operators acting in $(V \otimes V)$ and defined as:

$$
I_{j_{1} j_{2}}^{i_{1} i_{2}}=\delta_{j_{1}}^{i_{1}} \delta_{j_{2}}^{i_{2}}, \quad P_{j_{1} j_{2}}^{i_{1} i_{2}}=\delta_{j_{2}}^{i_{1}} \delta_{j_{1}}^{i_{2}}
$$

Using these operators one may define the symmetric $\mathbb{S C}$ and anti-symmetric $\mathbb{A} \mathbb{C}$ parts of the split Casimir operator $\hat{C}$ in the fundamental representation

$$
\begin{gathered}
\mathbb{S C}_{j_{1} j_{2}}^{i_{1} i_{2}}=\frac{1}{2}((I+P) \hat{C})_{j_{1} j_{2}}^{i_{2} i_{1}}=\frac{1}{2}\left(\hat{C}_{j_{1} j_{2}}^{i_{1} i_{2}}+\hat{C}_{j_{1} j_{2}}^{i_{2} i_{1}}\right) \\
\mathbb{A} \mathbb{C}_{j_{1} j_{2}}^{i_{1} i_{2}}=\frac{1}{2}((I-P) \hat{C})_{j_{1} j_{2}}^{i_{2} i_{1}}=\frac{1}{2}\left(\hat{C}_{j_{1} j_{2}}^{i_{1} i_{2}}-\hat{C}_{j_{1} j_{2}}^{i_{2} i_{1}}\right), \quad \hat{C}=\mathbb{S} \mathbb{C}+\mathbb{A} \mathbb{C} .
\end{gathered}
$$

Note that by definition we have

$$
P \cdot \hat{C}=\hat{C} \cdot P, \quad(I \pm P) \cdot \hat{C} \cdot(I \mp P)=0, \quad \mathbb{S} \mathbb{C} \cdot \mathbb{A} \mathbb{C}=0=\mathbb{A} \mathbb{C} \cdot \mathbb{S} \mathbb{C},
$$

and, in accordance with (4.1.1) and (4.1.2), one obtains

$$
\operatorname{Tr}_{12}(\mathbb{S} \mathbb{C})=-\operatorname{Tr}_{12}(\mathbb{A} \mathbb{C})=\frac{1}{2} \operatorname{dim} \mathfrak{g}
$$

Besides the standard Yang-Baxter equation for $R$ matrix (1.0.2), we also need the Yang-Baxter equation for the twisted $R$-matrix: $\check{R}(u) \equiv P R(u)$. This equation follows from (1.0.2) and is written in the form of the braid group relations

$$
\check{R}_{12}(u) \check{R}_{23}(u+v) \check{R}_{12}(v)=\check{R}_{23}(v) \check{R}_{12}(u+v) \check{R}_{23}(u)
$$


or in the components

$$
\check{R}(u)_{k_{1} k_{2}}^{i_{1} i_{2}} \check{R}(u+v)_{j_{2} l_{3}}^{k_{2} i_{3}} \check{R}(v)_{l_{1} l_{2}}^{k_{1} j_{2}}=\check{R}(v)_{k_{2} k_{3}}^{i_{2} i_{3}} \check{R}(u+v)_{l_{1} j_{2}}^{i_{1} k_{2}} \check{R}(u)_{l_{2} l_{3}}^{j_{2} k_{3}}
$$

Below we always require the unitarity condition

$$
\check{R}(u)_{k_{1} k_{2}}^{i_{1} i_{2}} \check{R}(-u)_{j_{1} j_{2}}^{k_{1} k_{2}}=\delta_{j_{1}}^{i_{1}} \delta_{j_{2}}^{i_{2}} \Rightarrow \quad \check{R}(u) \check{R}(-u)=P R(u) P R(-u)=1
$$

for the solutions of the Yang-Baxter equations (4.1.8).

\subsubsection{Split Casimir operator and $R$-matrix for the algebra $\mathfrak{g}_{2}$}

The dimension of the minimal fundamental (defining) representation of the Lie algebra $\mathfrak{g}_{2}$ is equal to 7 . It is known that the algebra $\mathfrak{g}_{2}$ is embedded into so(7) (see e.g. [6], [7]). This embedding can be constructed by using the definition of the algebra $\mathfrak{g}_{2}$ as the algebra of differentiations of the octonions. The procedure looks as follows [26]. We consider the algebra $\mathbb{O}$ of octonions with the generators $e_{0}=1, e_{i}(i=1, \ldots, 7)$ obeying the following multiplication rules:

$$
e_{i} \cdot e_{j}=-\delta_{i j}+f_{i j k} e_{k}, \quad i, j, k=1,2, \ldots, 7
$$

where the structure constants $f_{i j k}$ are the components of the completely anti-symmetric 3-rd rank tensor. The non-zero components (including index permutations) of this tensor are

$$
f_{123}=f_{145}=f_{176}=f_{246}=f_{257}=f_{347}=f_{365}=1 .
$$

Let $D$ be the differentiation of the algebra $\mathbb{O}$ :

$$
D(a \cdot b)=D(a) \cdot b+a \cdot D(b), \quad \forall a, b \in \mathbb{O}, \quad D(1)=0, \quad D\left(e_{i}\right)=e_{k} D_{k i},
$$

where $D_{i k}$ is the matrix of the differentiation operator. If we differentiate the relation (4.1.10), then using (4.1.12) we obtain

$$
D_{i j}=-D_{j i}, \quad D_{i m} f_{m j k}+D_{j m} f_{i m k}+D_{k m} f_{i j m}=0 .
$$

In other words, the matrix $D_{i j}$ is antisymmetric and, therefore, it belongs to the algebra $s o(7)$, while the second condition in (4.1.13) shows that the tensor $f_{i j k}$ is invariant under the action of the elements $D \in s o(7)$. This second condition produces 7 additional relations on the matrix $D_{i j}$

$$
\begin{gathered}
D_{26}+D_{15}=D_{73}, \quad D_{63}+D_{27}=D_{14}, \quad D_{17}+D_{24}=D_{35}, \quad D_{43}+D_{16}=D_{25}, \\
D_{13}+D_{64}=D_{57}, \quad D_{56}+D_{21}=D_{47}, \quad D_{23}+D_{45}=D_{67},
\end{gathered}
$$

which reduce the number of its independent components to 14. For example, one can express the components $D_{1 i}$ and $D_{23}$ through the other ones and expand the antisymmetric matrix $D$ over the remaining 14 free parameters to obtain the basis in the algebra $\mathfrak{g}_{2}$.

The useful identities for $f_{i j k}$ follow from the definition (4.1.11):

$$
\begin{gathered}
f_{i j k} f_{j k \ell}=6 \delta_{i \ell} \\
f_{i j k} f_{k \ell m} f_{m r i}=+3 f_{j \ell r} .
\end{gathered}
$$


The split Casimir operator (4.1.2) of the algebra $\mathfrak{g}_{2}$ in the minimal fundamental representation [7] acts in the reducible 49-dimensional space [7] $\times$ [7] which can be expanded in the irreducible representations as follows:

$$
[7] \times[7]=\mathbb{S}([7] \times[7])+\mathbb{A}([7] \times[7])=([1]+[27])+([7]+[14]) .
$$

Here the fundamental [7] and adjoint [14] representations embed into the antisymmetric part of [7] $\times[7]$, while the representations [1] and [27] compose the symmetric part of $[7] \times[7]$.

In the space of the representation [7] $\times[7]$ we define four operators

$$
(I)_{j_{1} j_{2}}^{i_{1} i_{2}}=\delta_{j_{1}}^{i_{1}} \delta_{j_{2}}^{i_{2}}, \quad(P)_{j_{1} j_{2}}^{i_{1} i_{2}}=\delta_{j_{2}}^{i_{1}} \delta_{j_{1}}^{i_{2}}, \quad(K)_{j_{1} j_{2}}^{i_{1} i_{2}}=\delta^{i_{1} i_{2}} \delta_{j_{1} j_{2}}, \quad(F)_{j_{1} j_{2}}^{i_{1} i_{2}}=f^{i_{1} i_{2} m} f_{m j_{1} j_{2}}
$$

invariant with respect to the action of the algebra $\mathfrak{g}_{2}$, i.e., for any linear combination $X$ of the operators (4.1.17) we have

$$
(a \otimes I+I \otimes a) \cdot X=X \cdot(a \otimes I+I \otimes a), \quad \forall a \in \mathfrak{g}_{2} .
$$

Using operators (4.1.17) one may construct four mutually orthogonal projectors [25]:

$$
\begin{array}{cl}
P^{[1]}=\frac{1}{7} K, \quad & P^{[7]}=\frac{1}{6} F, \quad P^{[27]}=\frac{1}{2}(I+P)-\frac{1}{7} K, \\
& P^{[14]}=\frac{1}{2}(I-P)-\frac{1}{6} F,
\end{array}
$$

which form the complete system $P^{[1]}+P^{[7]}+P^{[27]}+P^{[14]}=I$ and extract irreducible representations in the tensor product of two defining representations $[7] \times[7]$.

Note that the explicit form of the projector $P^{[7]}$ leads to the interpretation of the structure constants $f^{i_{1} i_{2} m}$ as Clebsch-Gordan coefficients describing the fusion of two fundamental representations [7] $\times[7]$ into one such representation [7].

The symmetric part $\mathbb{S} \mathbb{C}$ of the split Casimir operator is expressed through the invariant structures (4.1.17) as

$$
\mathbb{S C}=\frac{1}{6}(I+P)-\frac{1}{3} K
$$

while the projectors $\mathrm{P}^{(7)}$ and $\mathrm{P}^{(14)}$ are related to the antisymmetric part $\mathbb{A} \mathbb{C}$ of the split Casimir operator

$$
\mathrm{P}^{(7)}=-\mathbb{A} \mathbb{C}, \quad \mathrm{P}^{(14)}=\frac{1}{2}(I-P)+\mathbb{A} \mathbb{C} .
$$

Therefore, the split Casimir operator (4.1.2) for the algebra $\mathfrak{g}_{2}$ reads

$$
\hat{C}=\frac{1}{6}(I+P-2 K-F) \text {. }
$$

In addition, we have the following useful relation

$$
\hat{C}_{i_{2} j_{2}}^{i_{1} j_{1}}=-\left(T_{a}\right)^{i_{1} i_{2}} \otimes\left(T_{a}\right)_{j_{1} j_{2}}=-\left(P^{[14]}\right)^{i_{1} i_{2}}{ }_{j_{1} j_{2}} .
$$

Proposition 4.1.1 The characteristic identity for the operator $\hat{C}$ reads

$$
\hat{C}(\hat{C}-1 / 3)(\hat{C}+1)(\hat{C}+2)=0 \text {. }
$$


Proof. The spectral decomposition for the split Casimir operator (4.1.22) follows from the definitions of the projectors in (4.1.19)

$$
\hat{C}=\frac{1}{3} P^{[27]}-P^{[7]}-2 P^{(1)} \Rightarrow \hat{C} P^{[14]}=0 .
$$

Thus, the operator $\hat{C}$ has four eigenvalues $a_{1}=0, a_{2}=1 / 3, a_{3}=-1, a_{4}=-2$ (which correspond to the projectors $P^{[14]}, P^{[27]}, P^{[7]}, P^{[1]}$, respectively), which immediately leads to the identity (4.1.24).

For completeness, we give explicit formulas for the projectors $P_{a_{i}}$ in terms of the operator $\hat{C}$ :

$$
\begin{gathered}
P_{0}=-\frac{3}{2}(\hat{C}-1 / 3)(\hat{C}+1)(\hat{C}+2) \equiv P^{[14]}, \quad P_{1 / 3}=\frac{27}{28} \hat{C}(\hat{C}+1)(\hat{C}+2) \equiv P^{[27]} \\
P_{-1}=\frac{3}{4} \hat{C}(\hat{C}-1 / 3)(\hat{C}+2) \equiv P^{[7]}, \quad P_{-2}=-\frac{3}{14} \hat{C}(\hat{C}-1 / 3)(\hat{C}+1) \equiv P^{[1]}
\end{gathered}
$$

These formulas are obtained by means of identity (4.1.24) via the standard procedure.

The $\mathfrak{g}_{2}$-invariant solution $R(u)$ of the Yang-Baxter equation (1.0.2) in the defining representation was found in [25],[26]. The braid form of this solution is

$$
\check{R}(u)=\frac{1}{1-u}\left(I-u P-\frac{2 u}{(u-6)} K+\frac{u}{(u-4)} F\right) .
$$

Using formulas (4.1.19) we obtain the spectral decomposition of this solution

$$
\check{R}(u)=\frac{(u+1)(u+6)}{(u-1)(u-6)} P^{[1]}-\frac{(u+4)}{(u-4)} P^{[7]}-\frac{(u+1)}{(u-1)} P^{[14]}+P^{[27]},
$$

after which the fulfillment of the unitarity condition (4.1.9) for this $R$-matrix becomes evident.

Finally, the standard $\mathfrak{g}_{2}$-invariant $R$-matrix acquires the form [26], [28]

$$
\begin{gathered}
R(u)=P \check{R}(u)=\frac{(u+6)(u+1)}{(u-6)(u-1)} P^{[1]}+\frac{u+4}{u-4} P^{[7]}+\frac{u+1}{u-1} P^{[14]}+P^{[27]}= \\
=\frac{1}{u-1}\left(u-P+\frac{2 u}{(u-6)} K+\frac{u}{(u-4)} F\right) .
\end{gathered}
$$

Remarkably, this $\mathfrak{g}_{2}$-invariant solution of the Yang-Baxter equation can be rewritten as the rational function of the symmetric and antisymmetric parts of the split Casimir operator

$$
R(u)=\frac{(3 \mathbb{S} \mathbb{C}-u)}{(3 \mathbb{S} \mathbb{C}+u)} \cdot \frac{(3 \mathbb{A} \mathbb{C}-1-u)}{(3 \mathbb{A} \mathbb{C}-1+u)}
$$

\subsubsection{Split Casimir operator and $R$-matrix for the algebra $\mathfrak{f}_{4}$}

The minimal fundamental (defining) representation $T$ of the Lie algebra $\mathfrak{f}_{4}$ has dimension 26. In this representation the algebra $\mathfrak{f}_{4}$ can be embedded into the algebra $s o(26)$.

We follow the approaches of [31] and [32] to define the basis of the algebra $\mathfrak{f}_{4}$ in the representation $T$. For this, we consider the Jordan algebra $\mathbb{J}_{3}$ that consists of the hermitian $3 \times 3$ matrices with the elements: $o_{\alpha \beta}=\bar{o}_{\beta \alpha} \in \mathbb{O}(\alpha, \beta=1,2,3)$ :

$$
A=\left(\begin{array}{ccc}
x_{1} & o_{12} & o_{13} \\
\bar{o}_{12} & x_{2} & o_{23} \\
\bar{o}_{13} & \bar{o}_{23} & x_{3}
\end{array}\right), \quad x_{\alpha} \equiv o_{\alpha \alpha}=\bar{o}_{\alpha \alpha} \in \mathbb{R}
$$


Thus, the matrices $A$ are defined by three octonions $\bar{o}_{12}, \bar{o}_{13}, \bar{o}_{23}$ and three real numbers $x_{\alpha}$, which form the $(3 \cdot 8+3)=27$-dimensional real space. Multiplication in the algebra $\mathbb{J}_{3}$ is defined as $A \circ B \equiv \frac{1}{2}[A, B]_{+}\left(\forall A, B \in \mathbb{J}_{3}\right)$. It is easy to check that if $A$ and $B$ belong to $\mathbb{J}_{3}$, then $[A, B]_{+}$also belongs to $\mathbb{J}_{3}$.

Let us choose in $\mathbb{J}_{3}$ the basis $e_{0}=I_{3}, \mathrm{e}_{i}(i=1, \ldots, 26)$, where $I_{3}$ - is the $3 \times 3$ identity matrix, while $e_{i}$ are the basis elements in the space of traceless matrices in $\mathbb{J}_{3}$

$$
\begin{gathered}
\mathrm{e}_{1}=\left(\begin{array}{ccc}
1 & 0 & 0 \\
0 & -1 & 0 \\
0 & 0 & 0
\end{array}\right), \quad \mathrm{e}_{18}=\frac{1}{\sqrt{3}}\left(\begin{array}{ccc}
1 & 0 & 0 \\
0 & 1 & 0 \\
0 & 0 & -2
\end{array}\right), \quad \mathrm{e}_{1+a}=\left(\begin{array}{ccc}
0 & e_{a} & 0 \\
\bar{e}_{a} & 0 & 0 \\
0 & 0 & 0
\end{array}\right), \\
\mathrm{e}_{9+a}=\left(\begin{array}{ccc}
0 & 0 & e_{a} \\
0 & 0 & 0 \\
\bar{e}_{a} & 0 & 0
\end{array}\right), \quad \mathrm{e}_{18+a}=\left(\begin{array}{ccc}
0 & 0 & 0 \\
0 & 0 & e_{a} \\
0 & \bar{e}_{a} & 0
\end{array}\right), \quad(a=1,2, \ldots 8) .
\end{gathered}
$$

Here $e_{a} \in \mathbb{O}$ are the basis octonions (see (4.1.10) ). The nomalization of elements (4.1.31) is chosen so that $\operatorname{Tr}\left(\mathrm{e}_{i} \circ \mathrm{e}_{j}\right)=2 \delta_{i j}$ and the structure relations read

$$
\begin{aligned}
& \mathrm{e}_{i} \circ \mathrm{e}_{j} \equiv \frac{1}{2}\left[\mathrm{e}_{i}, \mathrm{e}_{j}\right]=\frac{2}{3} \delta_{i j} I_{3}-\frac{1}{2} d_{i, j, k} \mathrm{e}_{k}, \quad(i, j, k=1, \ldots, 26), \\
& \operatorname{Tr}\left(\mathrm{e}_{i} \circ \mathrm{e}_{j}\right)=2 \delta_{i j} .
\end{aligned}
$$

Here, the structure constants $d_{i, j, k}$ form the completely symmetric 3 -rd rank tensor and one can extract the explicit values of these constants from equation (4.1.32).

Let $D$ be the differentiation of the algebra $\mathbb{J}_{3}$ and $D\left(\mathrm{e}_{i}\right)=\mathrm{e}_{k} D_{k i}$. Acting by $D$ on the basic relation (4.1.32) we obtain

$$
D_{i j}=-D_{j i}, \quad D_{i m} d_{m j k}+D_{j m} d_{i m k}+D_{k m} d_{i j m}=0 .
$$

Thus, the matrices $D_{i j}$ of the differentiations of $\mathbb{J}_{3}$ belong to the algebra $s o(26)$ and their action on the tensor $d_{i j m}$ preserves it. The second condition in (4.1.33) reduces the number of independent matrices $D_{i j}$ to 52 , which is the dimension of the algebra $\mathfrak{f}_{4}$.

The split Casimir operator of the algebra $\mathfrak{f}_{4}$ in the representation $T$ acts in the reducible 676-dimensional representation [26] $\times$ [26] which can be expanded over the irreducible representations as follows:

$$
[26] \times[26]=\mathbb{S}([26] \times[26])+\mathbb{A}([26] \times[26])=([1]+[26]+[324])+([52]+[273]) .
$$

Here the antisymmetric part of [26] $\times[26]$ is decomposed into the representation [273] and the adjoint representation [52], while the symmetric part of [26] $\times$ [26] is decomposed into representations [1], [26] and [324].

In the space of the $[26] \times[26]$ representation one may defined five operators invariant respect to the algebra $\mathfrak{f}_{4}$

$$
\begin{gathered}
(I)_{j_{1} j_{2}}^{i_{1} i_{2}}=\delta_{j_{1}}^{i_{1}} \delta_{j_{2}}^{i_{2}}, \quad(P)_{j_{1} j_{2}}^{i_{1} i_{2}}=\delta_{j_{2}}^{i_{1}} \delta_{j_{1}}^{i_{2}}, \quad(K)_{j_{1} j_{2}}^{i_{1} i_{2}}=\delta^{i_{1} i_{2}} \delta_{j_{1} j_{2}} \\
(D)_{j_{1} j_{2}}^{i_{1} i_{2}}=d^{i_{1} i_{2} m} d_{j_{1} j_{2} m}, \quad(F)_{j_{1} j_{2}}^{i_{1} i_{2}}=T_{a}^{i_{1} i_{2}} T_{a j_{1} j_{2}}
\end{gathered}
$$

where $a=1,2, \ldots, 52$ and $T_{a i j} \equiv \delta_{i k}\left(T_{a}\right)^{k}{ }_{j}$ - are the generators of the algebra $\mathfrak{f}_{4}$ in the fundamental representation. With our definitions, the structure constant $d_{i j k}$ and generators $T_{a i j}$ obey the conditions:

$$
d_{i j k} d_{k \ell m} d_{m r i}=-8 d_{j \ell r}, \quad d^{i_{1} i_{2}, m} d_{i_{1} i_{2}, \ell}=\frac{56}{3} \delta_{\ell}^{m}, \quad \operatorname{Tr}\left(T_{a} T_{b}\right)=-\delta_{a b} .
$$


Using the operators (4.1.35), one may construct five mutually orthogonal projectors:

$$
\begin{gathered}
P^{[1]}=\frac{1}{26} K, \quad P^{(26)}=\frac{3}{56} D=\frac{1}{14}(I+P)-\frac{6}{7} \mathbb{S} \mathbb{C}-\frac{1}{14} K, \\
P^{[324]}=\frac{3}{7}\left((I+P)+2 \mathbb{S} \mathbb{C}+\frac{1}{13} K\right), \\
P^{[273]}=\frac{1}{2}(I-P)+2 \mathbb{A} \mathbb{C}, \quad P^{[52]}=F=-2 \mathbb{A} \mathbb{C},
\end{gathered}
$$

which form the complete system $P^{[1]}+P^{[26]}+P^{[52]}+P^{[273]}+P^{[324]}=I$ and which single out irreducible representations in the tensor product of two defining representations $[26] \times[26]$.

Note that symmetric and anti-symmetric parts of the split Casimir operator can be represented as

$$
\mathbb{S} \mathbb{C}=\frac{1}{12}\left(I+P-K-\frac{3}{4} D\right), \quad \mathbb{A} \mathbb{C}=-\frac{1}{2} F
$$

while the full split Casimir operator reads

$$
\widehat{C}=\mathbb{S} \mathbb{C}+\mathbb{A} \mathbb{C}=\frac{1}{12}(I+P-K)-\frac{1}{16} D-\frac{1}{2} F .
$$

Proposition 4.1.2 The split Casimir operator $\hat{C}$ for the algebra $\mathfrak{f}_{4}$ in the defining representation satisfies the characteristic identity

$$
\hat{C}(\hat{C}+1)(\hat{C}+2)(\hat{C}+1 / 2)(\hat{C}-1 / 6)=0 .
$$

Proof. The spectral decomposition of the split Casimir operator (4.1.39) follows from the definitions of the projectors in (4.1.37)

$$
\hat{C}=\frac{1}{6} P^{[324]}-P^{[26]}-2 P^{[1]}-\frac{1}{2} P^{[52]} \quad \Rightarrow \quad \hat{C} P^{[273]}=0 .
$$

Thus, the operator $\hat{C}$ has five eigenvalues $a_{1}=0, a_{2}=-1, a_{3}=-2, a_{4}=-1 / 2, a_{5}=1 / 6$, which immediately leads to the identity (4.1.40).

The spectral decomposition of the $\mathfrak{f}_{4}$-invariant solution $\check{R}(u)$ of the Yang-Baxter equation (4.1.8) in the defining representation was obtained in [26] and has the braid form

$$
\check{R}(u)=\frac{(u+9)(u+4)}{(u-9)(u-4)} P^{[1]}+\frac{(u+6)(u+1)}{(u-6)(u-1)} P^{[26]}-\frac{u+4}{u-4} P^{[52]}-\frac{u+1}{u-1} P^{[273]}+P^{[324]} .
$$

It is clear that the solution $\check{R}(u)(4.1 .42$ ) automatically obeys the unitarity condition.

Finally, the standard $\mathfrak{f}_{4}$-invariant $R$-matrix can be written in terms of the invariants (4.1.35) and acquires the form

$$
\begin{aligned}
& R(u)=P \check{R}(u)= \\
& =\frac{(u+9)(u+4)}{(u-9)(u-4)} P^{[1]}+\frac{(u+6)(u+1)}{(u-6)(u-1)} P^{[26]}+\frac{u+4}{u-4} P^{[52]}+\frac{u+1}{u-1} P^{[273]}+P^{[324]}= \\
& =\frac{1}{u-1}\left(u-P+\frac{u(u-1)}{(u-9)(u-4)} K+\frac{6 u}{(u-4)} F+\frac{3 u}{4(u-6)} D\right) .
\end{aligned}
$$

\footnotetext{
${ }^{2}$ There is a missprint in the form of the $\mathfrak{f}_{4}$-invariant solution presented in [26].
} 
Interestingly, this solution of the Yang-Baxter equation can be rewritten in the concise form as a rational function of the symmetric and antisymmetric parts of the split Casimir operator

$$
R(u)=\frac{\left(6 \mathbb{S C}^{\prime}-u\right)}{\left(6 \mathbb{S} \mathbb{C}^{\prime}+u\right)} \cdot \frac{\left(6 \mathbb{A} \mathbb{C}^{\prime}-1-u\right)}{\left(6 \mathbb{A} \mathbb{C}^{\prime}-1+u\right)}
$$

Here we introduced the notation $\mathbb{S C}^{\prime} \equiv \mathbb{S C}+\beta P^{[1]}, \mathbb{A} \mathbb{C}^{\prime} \equiv \mathbb{A} \mathbb{C}-\beta P^{[1]}$ and $\beta=1 / 2$, or $\beta=4 / 3$ (for both values of the parameter $\beta$ the operators (4.1.44) coincide in view of the characteristic identities for $\mathbb{A} \mathbb{C}$ and $\mathbb{S C}$ ).

\subsubsection{Split Casimir operator and $R$-matrix for the algebra $\mathfrak{e}_{6}$}

The algebra $\mathfrak{e}_{6}$, with dimension equal to 78 , has two inequivalent minimal fundamental representations [27] and [27]. We only consider the split Casimir operator of the algebra $\mathfrak{e}_{6}$ in the minimal fundamental representation which acts in the reducible [27] $\times$ [27]dimensional space. This space can be expanded in the following irreducible representations

$$
[27] \times[27]=\mathbb{S}([27] \times[27])+\mathbb{A}([27] \times[27])=\left([\overline{27}]+[351]_{1}\right)+\left([351]_{2}\right) .
$$

The mutually orthogonal projectors on these irreducible representations look as follow:

$$
P^{[27]}=\frac{1}{15}(I+P)-\frac{3}{5} \mathbb{S} \mathbb{C}, \quad P_{(1)}^{[351]}=-9 \mathbb{A} \mathbb{C}=\frac{1}{2}(I-P), \quad P_{(2)}^{[351]}=\frac{13}{30}(I+P)+\frac{3}{5} \mathbb{S} \mathbb{C},
$$

where $\mathbb{S C}$ and $\mathbb{A C}$ are respectively the symmetric and antisymmetric parts of the split Casimir operator $\hat{C}$ for the $\mathfrak{e}_{6}$ algebra in the representation [27].

Proposition 4.1.3 The operator $\hat{C}$ for $\mathfrak{e}_{6}$ algebra in the representation [27] satisfies the characteristic identity

$$
\left(\hat{C}+\frac{13}{9}\right)\left(\hat{C}+\frac{1}{9}\right)\left(\hat{C}-\frac{2}{9}\right)=0 .
$$

Proof. The spectral decomposition for the operator $\widehat{C}$ follows from the definitions of the projectors in (4.1.46):

$$
\widehat{C}=\frac{1}{9}\left(2 P_{(2)}^{[351]}-P_{(1)}^{[351]}-13 P^{[27]}\right) .
$$

Thus, the operator $\hat{C}$ has three eigenvalues $a_{1}=-\frac{13}{9}, a_{2}=-\frac{1}{9}, a_{3}=\frac{2}{9}$, which immediately leads to identity (4.1.47).

The $\mathfrak{e}_{6}$-invariant solution $\check{R}(u)$ of the Yang-Baxter equation (4.1.8) in the defining representation [27] has the form

$$
\check{R}(u)=\frac{u-4}{u+4} P^{[27]}+\frac{u+1}{u-1} P_{(2)}^{[351]}-P_{(1)}^{[351]} .
$$

It is clear that the solution $\check{R}(u)(4.1 .49)$ automatically obeys the unitarity condition.

Finally, the standard $\mathfrak{e}_{6}$-invariant $R$-matrix acquires the form [26]

$$
R(u)=P \check{R}(u)=\frac{u-4}{u+4} P^{[27]}+\frac{u+1}{u-1} P_{(2)}^{[351]}+P_{(1)}^{[351]} .
$$

The $\mathfrak{e}_{6}$-invariant solution $R(u)$ in (4.1.50) can be elegantly written as a rational function of $\widehat{C}$ (compare with (3.1.16)

$$
R(u)=-\frac{(3 \widehat{C}+1 / 3+u)}{(3 \widehat{C}+1 / 3-u)} .
$$




\subsubsection{Split Casimir operator and $R$-matrix for the algebra $\mathfrak{e}_{7}$}

The dimension of the exceptional algebra $\mathfrak{e}_{7}$ is 133 . The tensor product of its minimal 56-dimensional fundamental representations has the following expansion into irreducible ones

$$
[56] \times[56]=\mathbb{S}([56] \times[56])+\mathbb{A}([56] \times[56])=([133]+[1463])+([1]+[1539]) .
$$

The mutually orthogonal projectors on these irreducible representations read:

$$
\begin{aligned}
& P^{[1]}=-\frac{1}{112}(I-P)-\frac{3}{7} \mathbb{A} \mathbb{C}, \quad P^{[133]}=\frac{1}{16}(I+P)-\mathbb{S} \mathbb{C}, \\
& P^{[1463]}=\frac{7}{16}(I+P)+\mathbb{S} \mathbb{C}, \quad P^{[1539]}=\frac{57}{112}(I-P)+\frac{3}{7} \mathbb{A} \mathbb{C},
\end{aligned}
$$

where $\mathbb{S C}$ and $\mathbb{A C}$ are respectively the symmetric and antisymmetric parts of the split Casimir operator $\hat{C}$ for the $\mathfrak{e}_{7}$ algebra in the representation [56].

Proposition 4.1.4 The operator $\hat{C}$ for the $\mathfrak{e}_{7}$ algebra in the representation [56] satisfies the characteristic identity

$$
\left(\hat{C}-\frac{1}{8}\right)\left(\hat{C}+\frac{7}{8}\right)\left(\hat{C}+\frac{19}{8}\right)\left(\hat{C}+\frac{1}{24}\right)=0 .
$$

Proof. The following spectral decomposition for the split Casimir operator follows from the definitions of the projectors in (4.1.53)

$$
\widehat{C}=\mathbb{S C}+\mathbb{A} \mathbb{C}=\frac{1}{8}\left(P^{[1463]}-7 P^{[133]}\right)+\frac{1}{24}\left(-57 P^{[1]}-P^{[1539]}\right)
$$

Thus, the operator $\hat{C}$ has four eigenvalues $a_{1}=\frac{1}{8}, a_{2}=-\frac{7}{8}, a_{3}=-\frac{19}{8}, a_{4}=-\frac{1}{24}$, which immediately leads to identity (4.1.54).

The decomposition (4.1.52) of the antisymmetric part $\mathbb{A}([56] \times[56])$ of the tensor product $[56] \times[56]$ contains the singlet representation $[1]$. This means that the corresponding projector can be rewritten in the form

$$
\left(P^{[1]}\right)_{j_{1} j_{2}}^{i_{1} i_{2}}=-\frac{1}{56} J^{i_{1} i_{2}} J_{j_{1} j_{2}}, \quad J_{i k}=-J_{k i}, \quad J^{i k} J_{k j}=\delta_{j}^{i},
$$

where $J_{i k}$ and $J^{i k}$ are the invariant antisymmetric metrics. With the help of these metrics one may raise and lower indices of tensors. The existence of these metrics indicates that the $\mathfrak{e}_{7}$ algebra in the representation [56] is embedded as a subalgebra in the symplectic algebra $s p(56)$.

The $\mathfrak{e}_{7}$-invariant solution $\check{R}(u)$ of the Yang-Baxter equation (4.1.7) in the defining representation has the form

$$
\check{R}(u)=-\frac{(u-9)(u-5)}{(u+9)(u+5)} P^{[1]}+\frac{u-5}{u+5} P^{[133]}+\frac{u+1}{u-1} P^{[1463]}-P^{[1539]},
$$

The solution $\check{R}(u)$ (4.1.56) obviously obeys the unitarity condition.

Finally, the standard $\mathfrak{e}_{7}$-invariant $R$-matrix acquires the form [26]

$$
R(u)=P \check{R}(u)=\frac{(u-9)(u-5)}{(u+9)(u+5)} P^{[1]}+\frac{u-5}{u+5} P^{[133]}+\frac{u+1}{u-1} P^{[1463]}+P^{[1539]} .
$$


Note that this solution of the Yang-Baxter equation can also be written as a rational function of the "shifted" symmetric and antisymmetric parts of the split Casimir operator (cf. (4.1.44))

$$
R(u)=\frac{\left(u+6 \mathbb{S C}^{\prime}+1 / 4\right)}{\left(u-6 \mathbb{S} \mathbb{C}^{\prime}-1 / 4\right)} \cdot \frac{\left(u+6 \mathbb{A} \mathbb{C}^{\prime}\right)}{\left(u-6 \mathbb{A} \mathbb{C}^{\prime}\right)} .
$$

Here, we introduced the "shifted" symmetric and antisymmetric parts $\mathbb{S} \mathbb{C}^{\prime} \equiv \mathbb{S C}-\beta P^{[1]}$, $\mathbb{A} \mathbb{C}^{\prime} \equiv \mathbb{A} \mathbb{C}+\beta P^{[1]}$, where $\beta=37 / 4$, or $\beta=21 / 4$. For both values of the parameter $\beta$ the operators (4.1.57) coincide in view of the characteristic identities for $\mathbb{A} \mathbb{C}$ and $\mathbb{S} \mathbb{C}$.

\subsubsection{Algebra $\mathfrak{e}_{8}$}

The exceptional Lie algebra $\mathfrak{e}_{8}$ has dimension 248. Its minimal fundamental representation has also dimension 248 and it appears to be an adjoint representation of the algebra $\mathfrak{e}_{8}$. The split Casimir operators $\widehat{C}$ and their characteristic identities for all exceptional Lie algebras $\mathfrak{g}$ in the adjoint representation are discussed in the next section 4.2. So we postpone the consideration of the operator $\widehat{C}$ for the Lie algebra $\mathfrak{e}_{8}$ in the minimal fundamental (adjoint) representation to subsection 4.2.6.

It is known that there are no solutions of the Yang-Baxter equation ( $R$-matrices) for the simple Lie algebras in the adjoint representation besides the $s \ell$-series of the Lie algebras. This is the consequence of the fact that the adjoint representation of the simple Lie algebras $\mathfrak{g}$ (except $s \ell$ algebras) can not be extended to the representation of the Yangian $Y(\mathfrak{g})$ (see [33], 34]). However, the reducible representation $\mathfrak{g} \oplus \mathbb{C}$, which is a direct sum of the adjoint and trivial representations, can be extended to the representation of the Yangian $Y(\mathfrak{g})$. The $\mathfrak{g}$-invariant solution of the Yang-Baxter equation can be constructed just within such extended adjoint representation. For the extended adjoint (minimal fundamental) representation [248] of the algebra $\mathfrak{e}_{8}$, this solution has been constructed in [34] and [35]. The explicit form of this solution, written in the form of spectral decomposition over projectors, turns out to be rather cumbersome and we will not present it here. We assume that writing this solution in terms of the symmetrized $\mathbb{S} \mathbb{C}$ and antisymmetrized $\mathbb{A} \mathbb{C}$ parts of the operator $\widehat{C}$ will result in a more visual and compact formula.

\subsection{Characteristic identities for operator $\widehat{C}$ and invariant pro- jectors for exceptional Lie algebras in the adjoint represen- tations.}

In this section, we will find characteristic identities for the split Casimir operator $\widehat{C}$ in the adjoint representations for the exceptional algebras $\mathfrak{g}=\mathfrak{g}_{2}, \mathfrak{f}_{4}, \mathfrak{e}_{6}, \mathfrak{e}_{7}$ and $\mathfrak{e}_{8}$. As we noted at the end of theprevious section, the solutions of the Yang-Baxter equation that are invariant with respect to actions of exceptional Lie algebras in the adjoint representation do not exist, so this topic is not covered here.

\subsubsection{Basic definitions}

Let us define the normalization of the generators $X_{a}$ of the exceptional Lie algebra $\mathfrak{g}$ so that the Cartan-Killing metric (2.1.2) looks like

$$
\operatorname{Tr}\left(\operatorname{ad}\left(X_{a}\right) \operatorname{ad}\left(X_{d}\right)\right)=\sum_{c, b=1}^{\operatorname{dim} \mathfrak{g}}\left(C_{a}\right)_{b}^{c}\left(C_{d}\right)_{c}^{b}=-\delta_{a d} .
$$


where $\left(C_{d}\right)_{c}^{b} \equiv C_{d c}^{b}$ are the structure constants of the Lie algebra $\mathfrak{g}$. The split Casimir operator in the adjoint representation reads

$$
\left(\widehat{C}_{\mathrm{ad}}\right)_{b_{1} b_{2}}^{a_{1} a_{2}}=-\sum_{d}\left(C_{d}\right)_{b_{1}}^{a_{1}}\left(C_{d}\right)_{b_{2}}^{a_{2}}
$$

We will also need the identity $\mathbf{I}$ and permutation $\mathbf{P}$ operators defined as:

$$
\mathbf{I}_{b_{1} b_{2}}^{a_{1} a_{2}}=\delta_{b_{1}}^{a_{1}} \delta_{b_{2}}^{a_{2}}, \quad \mathbf{P}_{b_{1} b_{2}}^{a_{1} a_{2}}=\delta_{b_{2}}^{a_{1}} \delta_{b_{1}}^{a_{2}}
$$

together with the operator (2.2.2), which in the normalization (4.2.1)reads

$$
\mathbf{K}_{b_{1} b_{2}}^{a_{1} a_{2}}=\delta_{b_{1} b_{2}} \delta^{a_{1} a_{2}}
$$

In what follows, similarly to the previous consideration, it proved useful to define the symmetric $\widehat{C}_{+}$and antisymmetric $\widehat{C}_{-}$parts of the split Casimir operators in the adjoint representation

$$
\begin{aligned}
& \widehat{C}_{+}=\mathbf{P}_{+} \widehat{C}_{\mathrm{ad}} \Rightarrow\left(\widehat{C}_{+}\right)_{b_{1} b_{2}}^{a_{1} a_{2}}=\frac{1}{2}\left(\left(\widehat{C}_{\mathrm{ad}}\right)_{b_{1} b_{2}}^{a_{1} a_{2}}+\left(\widehat{C}_{\mathrm{ad}}\right)_{b_{1} b_{2}}^{a_{2} a_{1}}\right), \\
& \widehat{C}_{-}=\mathbf{P}_{-} \widehat{C}_{\mathrm{ad}} \Rightarrow\left(\widehat{C}_{-}\right)_{b_{1} b_{2}}^{a_{1} a_{2}}=\frac{1}{2}\left(\left(\widehat{C}_{\mathrm{ad}}\right)_{b_{1} b_{2}}^{a_{1} a_{2}}-\left(\widehat{C}_{\mathrm{ad}}\right)_{b_{1} b_{2}}^{a_{2} a_{1}}\right),
\end{aligned}
$$

where $\mathbf{P}_{ \pm}=\frac{1}{2}(\mathbf{I} \pm \mathbf{P})$.

\subsubsection{Algebra $\mathfrak{g}_{2}$}

The tensor product of two adjoint 14-dimensional representations of the algebra $\mathfrak{g}_{2}$ has the following decomposition into irreducible representations [4], 24]:

$$
[14] \times[14]=\mathbb{S}([14] \times[14])+\mathbb{A}([14] \times[14])=([1]+[27]+[77])+\left([14]+\left[77^{\star}\right]\right) .
$$

The dimensions of two representations appearing in the decomposition of $\mathbb{A}([14] \times[14])$ are given by (2.2.12). The antisymmetric $\widehat{C}_{-}$and symmetric $\widehat{C}_{+}$parts of the split Casimir operator $\widehat{C}_{\text {ad }}$ in the adjoint representation obey the following identities:

$$
\widehat{C}_{-}\left(\widehat{C}_{-}+\frac{1}{2}\right)=0, \quad \widehat{C}_{+}^{2}=-\frac{1}{6} \widehat{C}_{+}+\frac{5}{96}(\mathbf{I}+\mathbf{P}+\mathbf{K}) .
$$

Here the first identity is fulfilled for all simple Lie algebras, while the second one has been obtained by direct explicit calculations with the help of the Mathematica $^{T M}$ package (for details, see [36]).

Multiplying both parts of equation (4.2.7) by $\widehat{C}_{+}$and using the relations (2.2.4),(2.2.7), one can obtain

$$
\widehat{C}_{+}\left(\widehat{C}_{+}-\frac{1}{4}\right)\left(\widehat{C}_{+}+\frac{5}{12}\right)=-\frac{5}{96} \mathbf{K} \quad \Rightarrow \quad \widehat{C}_{+}\left(\widehat{C}_{+}+1\right)\left(\widehat{C}_{+}-\frac{1}{4}\right)\left(\widehat{C}_{+}+\frac{5}{12}\right)=0,
$$

where the second identity follows from the first ones after multiplying it by $\left(\widehat{C}_{+}+1\right)$ and taking into account (2.2.7).

The characteristic identity for the complete Casimir operator $\widehat{C}_{\text {ad }}=\widehat{C}_{+}+\widehat{C}_{-}$can be obtained from identities (4.2.7) and (4.2.8):

$$
\widehat{C}_{\mathrm{ad}}\left(\widehat{C}_{\mathrm{ad}}+1\right)\left(\widehat{C}_{\mathrm{ad}}+\frac{1}{2}\right)\left(\widehat{C}_{\mathrm{ad}}-\frac{1}{4}\right)\left(\widehat{C}_{\mathrm{ad}}+\frac{5}{12}\right)=0 .
$$


Using this identity together with the relation (4.2.7), one can find the projectors $\mathcal{P}_{\operatorname{dim}\left(V_{i}\right)} \equiv$ $\mathrm{P}_{\left(a_{i}\right)}$ on the eigenspaces $V_{i}$ of the operator $\widehat{C}_{\mathrm{ad}}$ with the eigenvalues $a_{i}=\left(-1,-\frac{1}{2},-\frac{5}{12}, \frac{1}{4}, 0\right)$ and, at the same time, on the representations (4.2.6). Finally, we derive

$$
\begin{aligned}
& \mathcal{P}_{14}=\mathrm{P}_{\left(-\frac{1}{2}\right)}=-2 \widehat{C}_{-}, \quad \mathcal{P}_{77}^{\star}=\mathrm{P}_{(0)}=\frac{1}{2}(\mathbf{I}-\mathbf{P})+2 \widehat{C}_{-}, \\
& \mathcal{P}_{1}=\mathrm{P}_{(-1)}=\frac{1}{14} \mathbf{K}, \quad \mathcal{P}_{27}=\mathrm{P}_{\left(-\frac{5}{12}\right)}=\frac{3}{16}(\mathbf{I}+\mathbf{P})-\frac{3}{2} \widehat{C}_{+}-\frac{15}{112} \mathbf{K}, \\
& \mathcal{P}_{77}=\mathrm{P}_{\left(\frac{1}{4}\right)}=\frac{5}{16}(\mathbf{I}+\mathbf{P})+\frac{3}{2} \widehat{C}_{+}+\frac{1}{16} \mathbf{K},
\end{aligned}
$$

where the first two and the last three projectors act on the antisymmetrized $\mathbf{P}_{-}\left(14^{\otimes 2}\right)$, and the symmetrized $\mathbf{P}_{+}\left(14^{\otimes 2}\right)$ parts of the representation $14^{\otimes 2}$, respectively. The dimensions $\operatorname{dim}\left(V_{i}\right)$ of the representations corresponding to the projectors (4.2.10) are calculated using formulas (2.2.10).

\subsubsection{Algebra $\mathfrak{f}_{4}$}

The exceptional Lie algebra $\mathfrak{f}_{4}$ has dimension 52 . The tensor product of its two adjoint 52-dimensional representations has the following decomposition into irreducible representations [4, 24]

$$
[52] \times[52]=\mathbb{S}([52] \times[52])+\mathbb{A}([52] \times[52])=([1]+[324]+[1053])+([52]+[1274]) .
$$

The dimensions of two representations in the decomposition of $\mathbb{A}([52] \times[52])$ are calculated by means of formulas (2.2.12). The antisymmetric $\widehat{C}_{-}$and symmetric $\widehat{C}_{+}$parts of the split Casimir operator $\widehat{C}$ for the algebra $\mathfrak{f}_{4}$ in the adjoint representation obey the following identities:

$$
\widehat{C}_{-}\left(\widehat{C}_{-}+\frac{1}{2}\right)=0, \quad \widehat{C}_{+}^{2}=-\frac{1}{6} \widehat{C}_{+}+\frac{5}{324}(\mathbf{I}+\mathbf{P}+\mathbf{K}) .
$$

Multiplying both parts of equation (4.2.12) by $\widehat{C}_{+}$and using the relations (2.2.4), (2.2.7), one obtains

$$
\widehat{C}_{+}\left(\widehat{C}_{+}-\frac{1}{9}\right)\left(\widehat{C}_{+}+\frac{5}{18}\right)=-\frac{5}{324} \mathbf{K} \quad \Rightarrow \quad \widehat{C}_{+}\left(\widehat{C}_{+}+1\right)\left(\widehat{C}_{+}-\frac{1}{9}\right)\left(\widehat{C}_{+}+\frac{5}{18}\right)=0,
$$

Thus, the characteristic identity for the full split Casimir operator $\widehat{C}_{\text {ad }}=\left(\widehat{C}_{+}+\widehat{C}_{-}\right)$reads

$$
\widehat{C}_{\text {ad }}\left(\widehat{C}_{\text {ad }}+1\right)\left(\widehat{C}_{\text {ad }}+\frac{1}{2}\right)\left(\widehat{C}_{\text {ad }}-\frac{1}{9}\right)\left(\widehat{C}_{\text {ad }}+\frac{5}{18}\right)=0 .
$$

Finally, the projectors $\mathcal{P}_{\operatorname{dim}\left(V_{i}\right)} \equiv \mathrm{P}_{\left(a_{i}\right)}$ onto the representations counted in the decomposition (4.2.11), or in other words, onto the representations acting in the eigenspaces $V_{i}$ of $\widehat{C}_{\text {ad }}$ with the eigenvalues $a_{i}=\left(-1,-\frac{1}{2},-\frac{5}{18}, \frac{1}{9}, 0\right)$ can be found to be

$$
\begin{aligned}
& \mathcal{P}_{52}=\mathrm{P}_{\left(-\frac{1}{2}\right)}=-2 \widehat{C}_{-}, \quad \mathcal{P}_{1274}=\mathrm{P}_{(0)}=\frac{1}{2}(\mathbf{I}-\mathbf{P})+2 \widehat{C}_{-}, \\
& \mathcal{P}_{1}=\mathrm{P}_{(-1)}=\frac{1}{52} \mathbf{K}, \quad \mathcal{P}_{324}=\mathrm{P}_{\left(-\frac{5}{18}\right)}=\frac{1}{7}(\mathbf{I}+\mathbf{P})-\frac{18}{7} \widehat{C}_{+}-\frac{5}{91} \mathbf{K}, \\
& \mathcal{P}_{1053}=\mathrm{P}_{\left(\frac{1}{9}\right)}=\frac{5}{14}(\mathbf{I}+\mathbf{P})+\frac{18}{7} \widehat{C}_{+}+\frac{1}{28} \mathbf{K} .
\end{aligned}
$$

\footnotetext{
${ }^{3}$ The second identity for the symmetric part of the Casimir operator here and for all other exceptional algebras below were obtained by direct explicit calculations with the help of the Mathematica $^{T M}$ package (for details, see [36]).
} 
The dimensions $\operatorname{dim}\left(V_{i}\right)$ of the representations corresponding to the projectors (4.2.15) are calculated using formulas (2.2.10).

\subsubsection{Algebra $\mathfrak{e}_{6}$}

The exceptional Lie algebra $\mathfrak{e}_{6}$ has dimension 78 . The tensor product of its two adjoint 78-dimensional representations has the following decomposition into irreducible representations [4, 24]:

$$
[78] \times[78]=\mathbb{S}([78] \times[78])+\mathbb{A}([52] \times[52])=([1]+[650]+[2430])+([78]+[2925])
$$

The dimensions of two representations in the decomposition of $\mathbb{A}([78] \times[78])$ are calculated by means of (2.2.12). The antisymmetric $\widehat{C}_{-}$and symmetric $\widehat{C}_{+}$parts of the split Casimir operator in the adjoint representation obey the following identities:

$$
\widehat{C}_{-}\left(\widehat{C}_{-}+\frac{1}{2}\right)=0, \quad \widehat{C}_{+}^{2}=-\frac{1}{6} \widehat{C}_{+}+\frac{1}{96}(\mathbf{I}+\mathbf{P}+\mathbf{K}) .
$$

From these identities, similarly to the previously considered cases of the $\mathfrak{g}_{2}$ and $\mathfrak{f}_{4}$ algebras, one obtains

$$
\widehat{C}_{+}\left(\widehat{C}_{+}+\frac{1}{4}\right)\left(\widehat{C}_{+}-\frac{1}{12}\right)=-\frac{1}{96} \mathbf{K} \quad \Rightarrow \quad \widehat{C}_{+}\left(\widehat{C}_{+}+1\right)\left(\widehat{C}_{+}+\frac{1}{4}\right)\left(\widehat{C}_{+}-\frac{1}{12}\right)=0,
$$

and, therefore,

$$
\widehat{C}_{\mathrm{ad}}\left(\widehat{C}_{\mathrm{ad}}+1\right)\left(\widehat{C}_{\mathrm{ad}}+\frac{1}{2}\right)\left(\widehat{C}_{\mathrm{ad}}+\frac{1}{4}\right)\left(\widehat{C}_{\mathrm{ad}}-\frac{1}{12}\right)=0 .
$$

Thus, the projectors $\mathcal{P}_{\operatorname{dim}\left(V_{i}\right)} \equiv \mathrm{P}_{\left(a_{i}\right)}$ on the representations listed in (4.2.16) and corresponding to the eigenvalues $a_{i}=\left(-1,-\frac{1}{2},-\frac{1}{4},-\frac{1}{12}, 0\right)$ read (cf. projectors in [4], Table 18.5)

$$
\begin{aligned}
& \mathcal{P}_{78}=\mathrm{P}_{\left(-\frac{1}{2}\right)}=-2 \widehat{C}_{-}, \quad \mathcal{P}_{2925}=\mathrm{P}_{(0)}=\frac{1}{2}(\mathbf{I}-\mathbf{P})+2 \widehat{C}_{-} \\
& \mathcal{P}_{1}=\mathrm{P}_{(-1)}=\frac{1}{78} \mathbf{K}, \quad \mathcal{P}_{650}=\mathrm{P}_{\left(-\frac{1}{4}\right)}=\frac{1}{8}(\mathbf{I}+\mathbf{P})-3 \widehat{C}_{+}-\frac{1}{24} \mathbf{K}, \\
& \mathcal{P}_{2430}=\mathrm{P}_{\left(\frac{1}{12}\right)}=\frac{3}{8}(\mathbf{I}+\mathbf{P})+3 \widehat{C}_{+}+\frac{3}{104} \mathbf{K} .
\end{aligned}
$$

These projectors are built using the standard method with the help of identity (4.2.19) and relations (4.2.17), (4.2.18). The dimensions $\operatorname{dim} V_{i}$ of the representations corresponding to the projectors (4.2.20) are calculated using formulas (2.2.10).

\subsubsection{Algebra $\mathfrak{e}_{7}$}

The exceptional Lie algebra $\mathfrak{e}_{7}$ has dimension 133 . The tensor product of its two adjoint 133-dimensional representations has the following decomposition into irreducible representations [4, 24]:

$$
[133] \times[133]=\mathbb{S}([133] \times[133])+\mathbb{A}([133] \times[133])=([1]+[1539]+[7371])+([133]+[8645])
$$


The dimensions of two representations in the decomposition of $\mathbb{A}([133] \times[133])$ are given by formula (2.2.12). The antisymmetric $\widehat{C}_{-}$and symmetric $\widehat{C}_{+}$parts of the split Casimir operator in the adjoint representation obey the following identities:

$$
\widehat{C}_{-}\left(\widehat{C}_{-}+\frac{1}{2}\right)=0, \quad \widehat{C}_{+}^{2}=-\frac{1}{6} \widehat{C}_{+}+\frac{1}{162}(\mathbf{I}+\mathbf{P}+\mathbf{K}) .
$$

From these identities we obtain

$$
\widehat{C}_{+}\left(\widehat{C}_{+}+\frac{2}{9}\right)\left(\widehat{C}_{+}-\frac{1}{18}\right)=-\frac{1}{162} \mathbf{K} \quad \Rightarrow \quad \widehat{C}_{+}\left(\widehat{C}_{+}+1\right)\left(\widehat{C}_{+}+\frac{2}{9}\right)\left(\widehat{C}_{+}-\frac{1}{18}\right)=0,
$$

and, therefore,

$$
\widehat{C}_{\mathrm{ad}}\left(\widehat{C}_{\mathrm{ad}}+1\right)\left(\widehat{C}_{\mathrm{ad}}+\frac{1}{2}\right)\left(\widehat{C}_{\mathrm{ad}}+\frac{2}{9}\right)\left(\widehat{C}_{\mathrm{ad}}-\frac{1}{18}\right)=0 .
$$

Finally, the projectors $\mathcal{P}_{\operatorname{dim}\left(V_{i}\right)} \equiv \mathrm{P}_{\left(a_{i}\right)}$ on the representations appearing in the decomposition (4.2.21) and corresponding to the eigenvalues $a_{i}=\left(-1,-\frac{1}{2},-\frac{2}{9}, \frac{1}{18}, 0\right)$ read

$$
\begin{aligned}
& \mathcal{P}_{133}=\mathrm{P}_{\left(-\frac{1}{2}\right)}=-2 \widehat{C}_{-}, \quad \mathcal{P}_{8645}=\mathrm{P}_{(0)}=\frac{1}{2}(\mathbf{I}-\mathbf{P})+2 \widehat{C}_{-} \\
& \mathcal{P}_{1}=\mathrm{P}_{(-1)}=\frac{1}{133} \mathbf{K}, \quad \mathcal{P}_{1539}=\mathrm{P}_{\left(-\frac{2}{9}\right)}=\frac{1}{10}(\mathbf{I}+\mathbf{P})-\frac{18}{5} \widehat{C}_{+}-\frac{1}{35} \mathbf{K}, \\
& \mathcal{P}_{7371}=\mathrm{P}_{\left(\frac{1}{18}\right)}=\frac{2}{5}(\mathbf{I}+\mathbf{P})+\frac{18}{5} \widehat{C}_{+}+\frac{2}{95} \mathbf{K} .
\end{aligned}
$$

The dimensions $\operatorname{dim} V_{i}$ of the representations related to the projectors (4.2.25) are calculated by formulas (2.2.10).

\subsubsection{Algebra $\mathfrak{e}_{8}$}

The exceptional Lie algebra $\mathfrak{e}_{8}$ has dimension 248. The tensor product of its two adjoint 248-dimensional representations has the following decomposition into irreducible representations [4, 24]:

$$
\begin{gathered}
{[248] \times[249]=\mathbb{S}([248] \times[248])+\mathbb{A}([248] \times[248])=} \\
=([1]+[3875]+[27000])+([248]+[30380]) .
\end{gathered}
$$

The dimensions of two representations in the decomposition of $\mathbb{A}([248] \times[248])$ are calculated by formula (2.2.12). The antisymmetric $\widehat{C}_{-}$and symmetric $\widehat{C}_{+}$parts of the split Casimir operator $\widehat{C}_{\text {ad }}$ in the adjoint representation obey the following identities:

$$
\widehat{C}_{-}\left(\widehat{C}_{-}+\frac{1}{2}\right)=0, \quad \widehat{C}_{+}^{2}=-\frac{1}{6} \widehat{C}_{+}+\frac{1}{300}(\mathbf{I}+\mathbf{P}+\mathbf{K}) .
$$

From these identities one can obtain

$$
\widehat{C}_{+}\left(\widehat{C}_{+}+\frac{1}{5}\right)\left(\widehat{C}_{+}-\frac{1}{30}\right)=-\frac{1}{300} \mathbf{K} \quad \Rightarrow \quad \widehat{C}_{+}\left(\widehat{C}_{+}+1\right)\left(\widehat{C}_{+}+\frac{1}{5}\right)\left(\widehat{C}_{+}-\frac{1}{30}\right)=0 .
$$

The characteristic identity for the full split Casimir operator $\widehat{C}_{\text {ad }}=\left(\widehat{C}_{+}+\widehat{C}_{-}\right)$reads

$$
\widehat{C}_{\mathrm{ad}}\left(\widehat{C}_{\mathrm{ad}}+1\right)\left(\widehat{C}_{\mathrm{ad}}+\frac{1}{2}\right)\left(\widehat{C}_{\mathrm{ad}}+\frac{1}{5}\right)\left(\widehat{C}_{\mathrm{ad}}-\frac{1}{30}\right)=0 .
$$


The projectors $\mathcal{P}_{\operatorname{dim}\left(V_{i}\right)} \equiv \mathrm{P}_{\left(a_{i}\right)}$ on the representations in the decomposition (4.2.26), which correspond to the eigenvalues $a_{i}=\left(-1,-\frac{1}{2},-\frac{1}{5}, \frac{1}{30}, 0\right)$, read

$$
\begin{aligned}
& \mathcal{P}_{248}=\mathrm{P}_{\left(-\frac{1}{2}\right)}=-2 \widehat{C}_{-}, \quad \mathcal{P}_{30380}=\mathrm{P}_{(0)}=\frac{1}{2}(\mathbf{I}-\mathbf{P})+2 \widehat{C}_{-} \\
& \mathcal{P}_{1}=\mathrm{P}_{(-1)}=\frac{1}{248} \mathbf{K}, \quad \mathcal{P}_{3875}=\mathrm{P}_{\left(-\frac{1}{5}\right)}=\frac{1}{14}(\mathbf{I}+\mathbf{P})-\frac{30}{7} \widehat{C}_{+}-\frac{1}{56} \mathbf{K} \\
& \mathcal{P}_{27000}=\mathrm{P}_{\left(\frac{1}{30}\right)}=\frac{3}{7}(\mathbf{I}+\mathbf{P})+\frac{30}{7} \widehat{C}_{+}+\frac{3}{217} \mathbf{K} .
\end{aligned}
$$

The dimensions $\operatorname{dim} V_{i}$ of the representations related to the projectors (4.2.30) are calculated by formulas (2.2.10).

\subsection{Universal characteristic identities and general comments}

In the adjoint representations the antisymmetric parts of the split Casimir operators $h C_{-}$ for all simple Lie algebras obey the same identity

$$
\widehat{C}_{-}\left(\widehat{C}_{-}+\frac{1}{2}\right)=0
$$

The symmetric parts of the split Casimir operators $\widehat{C}_{+}$in the adjoint representation for the exceptional Lie algebras obey identities (4.2.7), (4.2.12), (4.2.17), (4.2.22) and (4.2.27), which have a similar structure 4

$$
\widehat{C}_{+}^{2}=-\frac{1}{6} \widehat{C}_{+}+\mu(\mathbf{I}+\mathbf{P}+\mathbf{K})
$$

where the universal parameter $\mu$ is fixed as follows:

$$
\mu=\frac{5}{6(2+\operatorname{dim}(\mathfrak{g}))} .
$$

Note that identities (3.1.50) and (3.2.23) for the algebras $s \ell(3)$ and $s o(8)$ have the same structure.

From (4.3.2) one can obtain the universal characteristic identity on the symmetric part of the split Casimir operator $\widehat{C}_{+}$

$$
\widehat{C}_{+}\left(\widehat{C}_{+}+1\right)\left(\widehat{C}_{+}^{2}+\frac{1}{6} \widehat{C}_{+}-2 \mu\right) \equiv \widehat{C}_{+}\left(\widehat{C}_{+}+1\right)\left(\widehat{C}_{+}+\frac{\alpha}{2 t}\right)\left(\widehat{C}_{+}+\frac{\beta}{2 t}\right)=0,
$$

where we introduced the notation for two eigenvalues of the $\widehat{C}_{+}$:

$$
\frac{\alpha}{2 t}=\frac{1-\mu^{\prime}}{12}, \quad \frac{\beta}{2 t}=\frac{1+\mu^{\prime}}{12}, \quad \mu^{\prime}:=\sqrt{1+288 \mu}=\sqrt{\frac{\operatorname{dim} \mathfrak{g}+242}{\operatorname{dim} \mathfrak{g}+2}}
$$

These parameters are related as

$$
3(\alpha+\beta)=t
$$

\footnotetext{
${ }^{4}$ The universal formulae (4.3.2) was obtained in [4], eq. (17.10), under the assumption that $\widehat{C}_{+}^{2}$ is expressed as a linear combination of $\mathfrak{g}$-invariant operators $(\mathbf{I}+\mathbf{P}), \mathbf{K}$ and $\widehat{C}_{+}$. We explicitly checked this assumption for all exeptional Lie algebras.
} 
With the fixed value of the parameter $\alpha$, this relation defines the line of the exceptional Lie algebras on the $\beta, t$ plane (see eq.(5.1.15) below). Following [4], note that $\mu^{\prime}$ is a rational number only for a certain sequence of dimensions dim $\mathfrak{g}$. It turns out that this sequence is finite 5 :

$$
\begin{gathered}
\operatorname{dim} \mathfrak{g}=3,8,14,28,47,52,78,96,119,133,190,248,287,336 \\
484,603,782,1081,1680,3479
\end{gathered}
$$

which includes the dimensions $14,52,78,133,248$ of the exceptional Lie algebras $\mathfrak{g}_{2}, \mathfrak{f}_{4}, \mathfrak{e}_{6}, \mathfrak{e}_{7}, \mathfrak{e}_{8}$, and the dimensions 8 and 28 of the algebras $s \ell(3)$ and $s o(8)$, which are sometimes also referred to as exceptional. Thus, for these algebras, using (4.3.5), we calculate the values of the parameters $\frac{\alpha}{2 t}, \frac{\beta}{2 t}$ given in Table 4 .

Table 4.

\begin{tabular}{|c|c|c|c|c|c|c|c|}
\hline & $s \ell(3)$ & $s o(8)$ & $\mathfrak{g}_{2}$ & $\mathfrak{f}_{4}$ & $\mathfrak{e}_{6}$ & $\mathfrak{e}_{7}$ & $\mathfrak{e}_{8}$ \\
\hline$\frac{\alpha}{2 t}$ & $-1 / 3$ & $-1 / 6$ & $-1 / 4$ & $-1 / 9$ & $-1 / 12$ & $-1 / 18$ & $-1 / 30$ \\
\hline$\frac{\beta}{2 t}$ & $1 / 2$ & $1 / 3$ & $5 / 12$ & $5 / 18$ & $1 / 4$ & $2 / 9$ & $1 / 5$ \\
\hline
\end{tabular}

These values are in agreement with the formulas (3.1.51), (3.2.31), (4.2.8), (4.2.13), (4.2.18), (4.2.23), (4.2.28). Taking into account that $\widehat{C}_{-}$satisfies (2.2.5) and $\widehat{C}_{+}$satisfies (4.3.4), we obtain the following identities for the total split Casimir operator $\widehat{C}_{\mathrm{ad}}=\left(\widehat{C}_{+}+\widehat{C}_{-}\right)$in the case of the exceptional Lie algebras:

$$
\begin{aligned}
& \widehat{C}_{\mathrm{ad}}\left(\widehat{C}_{\mathrm{ad}}+\frac{1}{2}\right)\left(\widehat{C}_{\mathrm{ad}}+1\right)\left(\widehat{C}_{\mathrm{ad}}^{2}+\frac{1}{6} \widehat{C}_{\mathrm{ad}}-2 \mu\right)=0 \Rightarrow \\
& \widehat{C}_{\mathrm{ad}}\left(\widehat{C}_{\mathrm{ad}}+\frac{1}{2}\right)\left(\widehat{C}_{\mathrm{ad}}+1\right)\left(\widehat{C}_{\mathrm{ad}}+\frac{\alpha}{2 t}\right)\left(\widehat{C}_{\mathrm{ad}}+\frac{\beta}{2 t}\right)=0 .
\end{aligned}
$$

Here $\mu$ is defined in (4.3.3) and $\frac{\alpha}{2 t}, \frac{\beta}{2 t}$ are given in Table 4.

Remark. The sequence (4.3.6) contains dimensions $\operatorname{dim} \mathfrak{g}^{*}=(10 m-122+360 / m)$, $(m \in \mathbb{N})$ referring to the adjoint representations of the so-called $E_{8}$ family of algebras $\mathfrak{g}^{*}$; see [4], eq. (21.1). For these dimensions we have the relation $\mu^{\prime}=|(m+6) /(m-6)|$. Two numbers 47 and 119 from the sequence (4.3.6) do not belong to the sequence dim $\mathfrak{g}^{*}$. Thus, the interpretation of these two numbers as dimensions of some algebras is missing. Moreover, for values dim $\mathfrak{g}$ given in (4.3.6), using (4.3.5), one can calculate dimensions (3.3.9) of the corresponding representations $Y(\alpha)$ :

$$
\begin{gathered}
\operatorname{dim} V_{\left(-\frac{\alpha}{2 t}\right)}=\left\{5,27,77,300, \frac{14553}{17}, 1053,2430, \frac{48608}{13}, \frac{111078}{19}, 7371,15504,27000,\right. \\
\left.\frac{841279}{23}, \frac{862407}{17}, 107892, \frac{2205225}{13}, \frac{578151}{2}, 559911, \frac{42507504}{31}, \frac{363823677}{61}\right\}
\end{gathered}
$$

Since $\operatorname{dim} V_{\left(-\frac{\alpha}{2 t}\right)}$ should be integer, we conclude that no Lie algebras exist with dimensions 47, 96, 119, 287, 336, 603, 782, 1680,3479, for which we assume characteristic identity (4.3.4) and the trace formulas (2.2.10).

\footnotetext{
${ }^{5}$ We thank D.O.Orlov who proved the finiteness of this sequence.
} 


\section{Universal characteristic identities for operator $\widehat{C}$ for simple Lie algebras in the adjoint representa- tion and Vogel parameters}

In the previous sections, the projectors were constructed onto the spaces of irreducible subrepresentations in the representation $\mathrm{ad}^{\otimes 2}$ for all simple complex Lie algebras (Lie algebras of classical series $A_{n}, B_{n}, C_{n}, D_{n}$ and the exceptional Lie algebras). In all cases the construction was carried out by finding the characteristic identities for the split Casimir operators. In this regard, it should be noted that certain results of this work, namely the construction of projectors in terms of the split Casimir operator and finding their dimensions can be obtained by using the Vogel parameters $\alpha, \beta$ and $\gamma$, which were introduced in [11] (see also [12, 14]). The specific values of these parameters correspond to each simple complex Lie algebra. These values and the value of $t=(\alpha+\beta+\gamma)$ are given in Table 5 (see below). Since all universal formulas for the simple Lie algebras are written as homogeneous functions of the parameters $\alpha, \beta$ and $\gamma$, and these formulas are independent of all permutations of $\alpha, \beta, \gamma$ one can consider simple Lie algebras as points in the space $\mathbb{R P}^{3} / \mathbb{S}_{3}$. It is convenient to choose normalization in which one of the parameters is fixed, for example $\alpha=-2$, which is already done in Table 5 . Note that the data in the first six lines of Table 5 coincide with the data given in Table 3 of Section $\mathbf{3 . 3}$. We indicate the Vogel parameters for the algebras $s \ell(3)$ and $s o(8)$ in the separate lines of Table 5 , since the characteristic identities (3.1.50), (3.1.51) and (3.2.30), (3.2.27) for the symmetric part $\widehat{C}_{+}$of the split Casimir operator in the adjoint representations have the same order and the same structure as for the exceptional Lie algebras (cf. (4.3.2), (4.3.4)).

Table 5 .

\begin{tabular}{|c|c|c|c|c|c|c|c|c|}
\hline Type & Lie algebra & $\alpha$ & $\beta$ & $\gamma$ & $t$ & $-\frac{\alpha}{2 t}=\frac{1}{t}$ & $-\frac{\beta}{2 t}$ & $-\frac{\gamma}{2 t}$ \\
\hline$A_{n}$ & $s \ell(n+1)$ & -2 & 2 & $n+1$ & $n+1$ & $\frac{1}{n+1}$ & $-\frac{1}{n+1}$ & $-1 / 2$ \\
\hline$B_{n}$ & $s o(2 n+1)$ & -2 & 4 & $2 n-3$ & $2 n-1$ & $\frac{1}{2 n-1}$ & $-\frac{2}{2 n-1}$ & $-\frac{2 n-3}{2(2 n-1)}$ \\
\hline$C_{n}$ & $s p(2 n)$ & -2 & 1 & $n+2$ & $n+1$ & $\frac{1}{n+1}$ & $-\frac{1}{2(n+1)}$ & $-\frac{n+2}{2(n+1)}$ \\
\hline$D_{n}$ & $s o(2 n)$ & -2 & 4 & $2 n-4$ & $2 n-2$ & $\frac{1}{2 n-2}$ & $-\frac{1}{n-1}$ & $-\frac{n-2}{n-1}$ \\
\hline$A_{2}$ & $s \ell(3)$ & -2 & 2 & 3 & 3 & $1 / 3$ & $-1 / 3$ & $-1 / 2$ \\
\hline$D_{4}$ & $s o(8)$ & -2 & 4 & 4 & 6 & $1 / 6$ & $-1 / 3$ & $-1 / 3$ \\
\hline$G_{2}$ & $\mathfrak{g}_{2}$ & -2 & $10 / 3$ & $8 / 3$ & 4 & $1 / 4$ & $-5 / 12$ & $-1 / 3$ \\
\hline$F_{4}$ & $\mathfrak{f}_{4}$ & -2 & 5 & 6 & 9 & $1 / 9$ & $-5 / 18$ & $-1 / 3$ \\
\hline$E_{6}$ & $\mathfrak{e}_{6}$ & -2 & 6 & 8 & 12 & $1 / 12$ & $-1 / 4$ & $-1 / 3$ \\
\hline$E_{7}$ & $\mathfrak{e}_{7}$ & -2 & 8 & 12 & 18 & $1 / 18$ & $-2 / 9$ & $-1 / 3$ \\
\hline$E_{8}$ & $\mathfrak{e}_{8}$ & -2 & 12 & 20 & 30 & $1 / 30$ & $-1 / 5$ & $-1 / 3$ \\
\hline
\end{tabular}

It proved useful to split the tensor product of two adjoint representations into the symmetric and antisymmetric parts

$$
\operatorname{ad} \otimes \operatorname{ad}=\mathbb{S}(\operatorname{ad} \otimes \mathrm{ad})+\mathbb{A}(\operatorname{ad} \otimes \operatorname{ad})
$$

In the general case of the Lie algebras of the classical series 6 , the symmetric part $\mathbb{S}\left(\operatorname{ad}^{\otimes 2}\right)$ decomposes into 4 irreducible representations: a singlet, denoted as $\mathrm{X}_{0}$, with zero eigenvalue of the quadratic Casimir operator $C_{(2)}$ (which corresponds to the eigenvalue $(-1)$

\footnotetext{
${ }^{6}$ The algebras $s \ell(3)$ and $s o(8)$ are exeptional cases.
} 
for the split operator $\widehat{C}$ ), and 3 representations which we denote as $Y_{2}(\alpha), Y_{2}(\beta), Y_{2}(\gamma)$. Their dimensions, as well as the corresponding values $c_{(2)}^{(\lambda)}$ and $\hat{c}_{(2)}^{(\lambda)}$ (here $\lambda=\mu, \mu^{\prime}, \mu^{\prime \prime}$ are the highest weights of the representations $\left.Y_{2}(\alpha), Y_{2}(\beta), Y_{2}(\gamma)\right)$ of the quadratic Casimir operator $C_{(2)}$ (defined in (2.1.4) ) and split Casimir operator $\widehat{C}$ are equal to:

$$
\begin{aligned}
& \operatorname{dim} Y_{2}(\alpha)=\operatorname{dim} V_{\left(-\frac{\alpha}{2 t}\right)}, \quad c_{(2)}^{(\mu)}=2-\frac{\alpha}{t}, \quad \hat{c}_{(2)}^{(\mu)}=-\frac{\alpha}{2 t}, \\
& \operatorname{dim} Y_{2}(\beta)=\operatorname{dim} V_{\left(-\frac{\beta}{2 t}\right)}, \quad c_{(2)}^{\left(\mu^{\prime}\right)}=2-\frac{\beta}{t}, \quad \hat{c}_{(2)}^{\left(\mu^{\prime}\right)}=-\frac{\beta}{2 t}, \\
& \operatorname{dim} Y_{2}(\gamma)=\operatorname{dim} V_{\left(-\frac{\gamma}{2 t}\right)}, \quad c_{(2)}^{\left(\mu^{\prime \prime}\right)}=2-\frac{\gamma}{t}, \quad \hat{c}_{(2)}^{\left(\mu^{\prime \prime}\right)}=-\frac{\gamma}{2 t} .
\end{aligned}
$$

where the explicit expressions for $\operatorname{dim} V_{\left(-\frac{\alpha}{2 t}\right)}, \operatorname{dim} V_{\left(-\frac{\beta}{2 t}\right)}, \operatorname{dim} V_{\left(-\frac{\gamma}{2 t}\right)}$ are given in (3.3.9)(3.3.11) and the eigenvalues $c_{(2)}^{(\lambda)}$ and $\hat{c}_{(2)}^{(\lambda)}$ of the operators $C_{(2)}$ and $\widehat{C}$ are related by the condition (2.3.6):

$$
\hat{c}_{(2)}^{(\lambda)}=\frac{1}{2}\left(c_{(2)}^{(\lambda)}-2 c_{(2)}^{\mathrm{ad}}\right)=\frac{1}{2} c_{(2)}^{(\lambda)}-1
$$

The eigenvalues $\hat{c}_{(2)}^{(\lambda)}$ of the operator $\widehat{C}$ on the representations $Y_{2}(\alpha), Y_{2}(\beta), Y_{2}(\gamma)$ in $\mathbb{S C}(\mathrm{ad} \times \mathrm{ad})$ are presented in three last columns of Table 5 . Therefore, taking into account that $\widehat{C}_{+}$has four eigenvalues $\left(-1,-\frac{\alpha}{2 t},-\frac{\beta}{2 t},-\frac{\gamma}{2 t}\right)$ and $\widehat{C}_{-}$has two eigenvalues $\left(0,-\frac{1}{2}\right)$, the generic characteristic identity for the split Casimir operator reads

$$
\widehat{C}_{\mathrm{ad}}\left(\widehat{C}_{\mathrm{ad}}+\frac{1}{2}\right)\left(\widehat{C}_{\mathrm{ad}}+1\right)\left(\widehat{C}_{\mathrm{ad}}+\frac{\alpha}{2 t}\right)\left(\widehat{C}_{\mathrm{ad}}+\frac{\beta}{2 t}\right)\left(\widehat{C}_{\mathrm{ad}}+\frac{\gamma}{2 t}\right)=0 .
$$

In the case of the $s \ell(N)$ algebras, the eigenvalue $(-1 / 2)$ of the operator $\widehat{C}_{\text {ad }}$ is doubly degenerated, since $\frac{\gamma}{2 t}=1 / 2$; therefore, in identity (5.1.14) one should keep only one factor $\left(\widehat{C}_{\mathrm{ad}}+\frac{1}{2}\right)$ of two (compare the identities (3.1.35) and (5.1.14) $)$.

We now turn to the discussion of the case of the exceptional Lie algebras. Note that all exceptional Lie algebras are distinguished in Table 5 by the value of the parameter $-\gamma /(2 t)$ equals to $-1 / 3$ (all other parameters of the exceptional Lie algebras in Table 5 are in agreement with the parameters listed in Table 4 of Section 4.3). Thus, all exceptional Lie algebras in the three-dimensional space of the Vogel parameters $(\alpha, \beta, \gamma)$ lie in the plane $\alpha=-2$ on the line:

$$
3 \gamma=2 t \quad \Rightarrow \quad \gamma=2 \beta-4 \text {. }
$$

We chose the coordinates $(\beta, \gamma)$ on this plane. When the condition (5.1.15) is fulfilled, the dimension (3.3.11), (5.1.12) of the space of the representation $Y_{2}(\gamma)$ is zero in view of the factor $(3 \gamma-2 t)$ in the numerator of (3.3.11). So the corresponding projector $\mathrm{P}_{\left(-\frac{\gamma}{2 t}\right)}$ on this space is also equal to zero and the parameter $-\gamma /(2 t)$ cannot be an eigenvalue of $\widehat{C}_{\text {ad }}$ (in the case of the non-exceptional Lie algebras, this parameter is the eigenvalue of the operator $\widehat{C}_{\text {ad }}$ on the representation $Y_{2}(\gamma)$; see Subsection 3.3). In this case, in the general characteristic identity (5.1.14) for the operator $\widehat{C}_{\text {ad }}=(\operatorname{ad} \otimes \operatorname{ad})(\widehat{C})$, the last factor $\left(\widehat{C}_{\text {ad }}+\frac{\gamma}{2 t}\right)$ will be absent and the universal characteristic identity coincides with (4.3.8):

$$
\widehat{C}_{\mathrm{ad}}\left(\widehat{C}_{\mathrm{ad}}+\frac{1}{2}\right)\left(\widehat{C}_{\mathrm{ad}}+1\right)\left(\widehat{C}_{\mathrm{ad}}+\frac{\alpha}{2 t}\right)\left(\widehat{C}_{\mathrm{ad}}+\frac{\beta}{2 t}\right)=0 .
$$

As we showed in Subsection 4.3. identity (5.1.16) for the values of the parameters $\alpha, \beta$ given in Table 4 and Table 5 exactly reproduces the characteristic identities (4.2.9), 
(4.2.14), (4.2.19), (4.2.24) and (4.2.29) for the split Casimir operator $\widehat{C}_{\text {ad }}$ in the case of the exceptional Lie algebras. Note that both algebras $s o(8)$ and $s \ell(3)$ (for the latter one has to replace the parameters $\beta \leftrightarrow \gamma$ ) lie on the line (5.1.15) and the characteristic identities (3.2.27) and (3.1.52) are also given by the generic formula (5.1.16). Indeed, for the algebra $s \ell(3)$ we have $\frac{\gamma}{2 t}=\frac{1}{2}$; therefore, the eigenvalue $(-1 / 2)$ of the operator $\widehat{C}_{\text {ad }}$ is doubly degenerated and one of the factors $\left(\widehat{C}_{\mathrm{ad}}+1 / 2\right)$ in (5.1.16) must be omitted. Wherein, for the algebra $s o(8)$ both parameters $\frac{\beta}{2 t}$ and $\frac{\gamma}{2 t}$ are equal to the critical value $\frac{1}{3}$, which gives zero in denominators of the expressions (3.3.10), (5.1.11) and (3.3.11), (5.1.12) for the dimensions $\operatorname{dim} V_{\left(-\frac{\beta}{2 t}\right)}$ and $\operatorname{dim} V_{\left(-\frac{\gamma}{2 t}\right)}$ of the representations $Y_{2}(\beta)$ and $Y_{2}(\gamma)$. However, these zeros are canceled with zeros coming from the terms $(3 \beta-2 t)$ and $(3 \gamma-2 t)$ in the numerators of the expressions for $\operatorname{dim} V_{\left(-\frac{\beta}{2 t}\right)}$, $\operatorname{dim} V_{\left(-\frac{\gamma}{2 t}\right)}$ and these dimensions turn out to be 35, which is consistent with (3.2.32). Since the eigenvalue $-\frac{\beta}{2 t}=-\frac{\gamma}{2 t}=-\frac{1}{3}$ of the operator $\widehat{C}_{\text {ad }}$ is doubly degenerated, we must omit one of the factors $\left(\widehat{C}_{\text {ad }}+1 / 3\right)$ in (5.1.14) and this identity is transformed into identity (5.1.16).

The antisymmetric part $\mathbb{A}(\mathrm{ad} \otimes \mathrm{ad})$ decomposes for all simple Lie algebras into a direct sum of two terms $X_{1}$ and $X_{2}$ (see Section 2.2), one of which $X_{1}$ is the adjoint representation ad with the value of the quadratic Casimir $c_{(2)}^{(\mathrm{ad})}=1$, and the other representation $\mathbf{X}_{2}$ has the value of the quadratic Casimir $c_{(2)}^{\left(\mathrm{X}_{2}\right)}=2$. The representation $\mathrm{X}_{2}$ is reducible for the case of algebras $s \ell(N)$ (see Subsection 3.1.2) and irreducible for all other simple Lie algebras. The dimension of the representations $\mathrm{X}_{1}, \mathrm{X}_{2}$ and the corresponding eigenvalues $\hat{c}_{(2)}^{(\mathrm{ad})}$ and $\hat{c}_{(2)}^{\left(\mathrm{X}_{2}\right)}$ are equal to $(\mathrm{cf} .(2.2 .12))$

$$
\begin{gathered}
\operatorname{dim} \mathbf{X}_{1}=\operatorname{dim} \mathfrak{g}, \quad \hat{c}_{(2)}^{(\mathrm{ad})}=-1 / 2, \\
\operatorname{dim} \mathbf{X}_{2}=\frac{1}{2} \operatorname{dim} \mathfrak{g}(\operatorname{dim} \mathfrak{g}-3), \quad \hat{c}_{(2)}^{\left(\mathrm{X}_{2}\right)}=0 .
\end{gathered}
$$

The values $\hat{c}_{(2)}^{(\mathrm{ad})}$ and $\hat{c}_{(2)}^{\left(\mathrm{X}_{2}\right)}$ agree with the characteristic identity (2.2.5) for the antisymmetrized part of $\widehat{C}_{-}$, which is valid for all simple Lie algebras.

\section{Acknowledgements}

The authors are thankful to O.V. Ogievetsky who draw our attention to the relation of the Vogel parametrization and characteristic identities of the split Casimir operator in the adjoint representation and to P. Cvitanović, R.L.Mkrtchyan, M.A.Vasiliev for useful comments. We are thankful to D. Lezin for the help with calculations of the projectors (3.1.47) at the initial stage. The authors are also grateful to D.O. Orlov and N.A. Tyurin for the explanation of the methods for solving the nonlinear Diophantine equations.

\section{References}

[1] V.Chari and A.N.Pressley, A guide to quantum groups. Cambridge university press (1995).

[2] Z.Ma, Yang-Baxter equation and quantum enveloping algebras. World Scientific (1993). 
[3] V.G.Drinfeld, Quantum groups, Journal of Soviet mathematics, 41(2), (1988) 898.

[4] P. Cvitanović, Birdtracks, Lie's, and Exceptional Groups, Princeton; Oxford: Princeton University Press (2008); http://cns.physics.gatech.edu/grouptheory/chapters/draft.pdf

[5] H. Weyl, The Classical Groups: Their Invariants and Representations, Princeton University Press (1997).

[6] H. Georgi, Lie algebras in particle physics, Westview press, Advanced Book Program, 1999.

[7] A.P. Isaev, V.A. Rubakov, Theory of groups and symmetries I. Finite groups, Lie groups and Lie algebras. World Scientific (2018).

[8] A.P. Isaev, V.A. Rubakov, Theory of groups and symmetries II. Representations of groups and Lie algebras, applications. World Scientific (2020).

[9] I.Todorov, and L.Hadjiivanov, Quantum groups and braid group statistics in conformal current algebra models, Vitória: EDUFES (2010); CERN preprint No. CERNPH-TH-2009-050.

[10] P. Deligne, La série exceptionnelle des groupes de Lie. C. R. Acad. Sci. 322 (1996), 321-326.

[11] P. Vogel, The universal Lie algebra, preprint (1999).

[12] J.M. Landsberg, L. Manivel, A universal dimension formula for complex simple Lie algebras Adv. Math. 201 (2006), 379-407

[13] R.L. Mkrtchyan, A.N. Sergeev, and A.P. Veselov, Casimir eigenvalues for universal Lie algebra, Journal of Mathematical Physics 53, (2012) 102106.

[14] R.L. Mkrtchyan and A.P. Veselov, Universality in Chern-Simons theory, Journal of High Energy Physics, 2012(8) 153.

[15] A. Mironov, R. Mkrtchyan, and A. Morozov, On universal knot polynomials, JHEP, 2016, No. 2, 78 (2016).

[16] R.L.Mkrtchyan, The equivalence of $S p(2 N)$ and $S O(-2 N)$ gauge theories, Phys.Lett. 105B (1981) 174-176.

[17] R.L. Mkrtchyan and A.P.Veselov, On duality and negative dimensions in the theory of Lie groups and symmetric spaces, J. Math. Phys. 52 (2011) 083514.

[18] A. Molev, Sugawara operators for classical Lie algebras, Mathematical Surveys and Monographs Vol.229, AMS (2018).

[19] R.L. Mkrtchyan, On the road map of Vogel's plane, Lett. Math. Phys., 106(1) (2016) 57-79.

[20] H.M. Khudaverdian and R.L. Mkrtchyan, Diophantine equations, Platonic solids, McKay correspondence, equivelar maps and Vogel's universality, Journal of Geom. and Phys., 114 (2017) 85-90. 
[21] A.P. Isaev, and A.A. Provorov, Projectors on invariant subspaces of representations $\mathrm{ad}^{\otimes 2}$ of Lie algebras $s o(N)$ and $s p(2 r)$ and Vogel parametrization, TMPh in press, e-Print: 2012.00746 [math-ph].

[22] R. Feger, and Th.W. Kephart, LieART - A Mathematica Application for Lie Algebras and Representation Theory, arXiv:1206.6379 (2012)

[23] A. Bincer, Lie Groups And Lie Algebras, Oxford: Oxford University Press, (2013), 89-92.

[24] N. Yamatsu, Finite-Dimensional Lie Algebras and Their Representations for Unified Model Building, arXiv:1511.08771 [hep-ph].

[25] E.I.Ogievetsky, Factorised S-matrix with $G_{2}$ symmetry, J.Phys. G: Nucl.Phys. 12 (1986) L105-L108.

[26] E.Ogievetsky, P.Wiegmann, Factorized S-matrix and the Bethe Ansatz for Simple Lie Groups, Phys.Lett. 168B No 4 (1986) 360.

[27] N.J.MacKay, Rational R-matrices in irreducible representations, J. of Phys. A: Math. and Gen. 24.17 (1991)4017;

N.J.MacKay and A.Taylor, Rational R-matrices, centralizer algebras and tensor identities for $e_{6}$ and $e_{7}$ exceptional families of Lie algebras, J. of Math. Phys. 48.10 (2007) 103507.

[28] A.Kuniba, Quantum R-matrix for $G_{2}$ and solvable 175-vertex model, J.Phys. A: Math.Gen. 23 (1990) 1349-1362.

[29] S.M.Sergeev, Spectral decompositions of R-matrices for exceptional Lie algebras, IHEP Preprint 90-163, Protvino, 1990.

[30] G.W.Delius, M.D.Gould and Y.Z.Zhang, On the construction of trigonometric solutions of the Yang-Baxter equation, Nucl.Phys. B432 1-2 (1994)377-403.

[31] M.M.Postnikov, Lie Groups and Lie Algebras. Lectures in Geometry, Semester V, Editorial URSS (1994).

[32] F.Bernardoni, S.L.Cacciatori, B.L.Cerchiai, and A.Scotti, Mapping the geometry of the $F_{4}$ group. Advances in Theoretical and Mathematical Physics, 12(4) (2008) 889-944.

[33] V.G. Drinfeld, Hopf algebras and the quantum Yang-Baxter equations, Dokl. Akad. Nauk. SSSR 283 (1985) (Russian), Soviet Math. Dokl. 32 (1985), 254-258.

[34] V.Chari and A.Pressley, Fundamental representations of Yangians and singularities of $R$-matrices, J. reine angew. Math., 417 (1991), 87-128

[35] K.Koepsell, H.Nicolai, and H.Samtleben, On the Yangian Y(e $\left.\mathfrak{e}_{8}\right)$ quantum symmetry of maximal supergravity in two dimensions, JHEP 04 (1999) 023, hep-th/9903111.

[36] S. Wolfram, Mathematica, A System for Doing Mathematics by Computer, (Addison-Wesley Publishing Company, Inc.) 1991. 